INTERNATIONAL MONETARY FUND
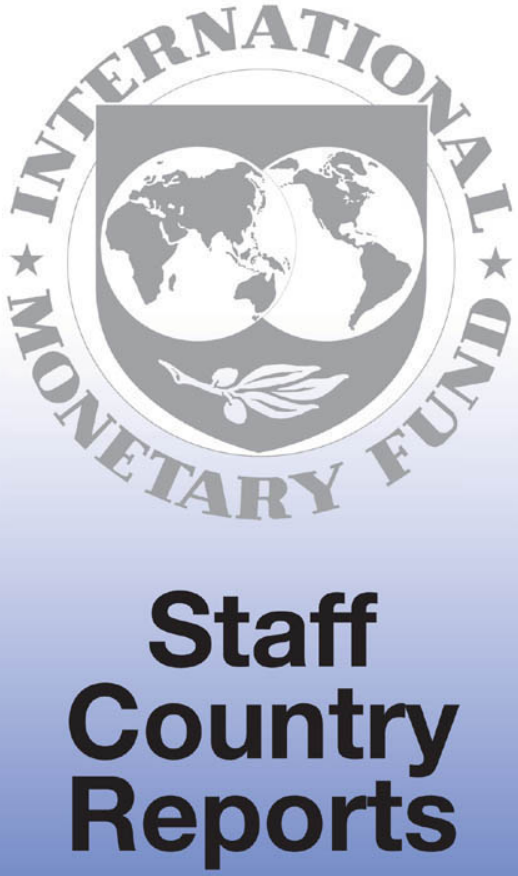


\section{Albania: Second Review Under the Three-Year Arrangement Under the Poverty Reduction and Growth Facility-Staff Report; Staff Statement; Press Release on the Executive Board Discussion; and Statement by the Executive Director for Albania}

In the context of the second review under the three-year arrangement under the Poverty Reduction and Growth Facility, the following documents have been released and are included in this package:

- the staff report for the second review under the three-year arrangement under the Poverty Reduction and Growth Facility, prepared by a staff team of the IMF, following discussions that ended on April 29, 2003, with the officials of Albania on economic developments and policies. Based on information available at the time of these discussions, the staff report was completed on June 17, 2003. The views expressed in the staff report are those of the staff team and do not necessarily reflect the views of the Executive Board of the IMF.

- a staff statement of July 2, 2003 updating information on recent developments.

- a Press Release summarizing the views of the Executive Board as expressed during its July 2, 2003 discussion of the staff report that completed the review.

- a statement by the Executive Director for Albania.

The document(s) listed below have been or will be separately released

Joint Staff Assessment of the Poverty Reduction Strategy Paper Progress Report

The policy of publication of staff reports and other documents allows for the deletion of market-sensitive information.

To assist the IMF in evaluating the publication policy, reader comments are invited and may be sent by e-mail to publicationpolicy(a)imforg.

Copies of this report are available to the public from

International Monetary Fund * Publication Services $70019^{\text {th }}$ Street, N.W. - Washington, D.C. 20431

Telephone: (202) 623-7430 - Telefax: (202) 623-7201

E-mail: publications@imf.org - Internet: http://www.imf.org

Price: $\$ 15.00$ a copy

\section{International Monetary Fund Washington, D.C.}


INTERNATIONAL MONETARY FUND

\author{
ALBANIA \\ Second Review Under the Three-YearArrangement \\ Under the Poverty Reduction and Growth Facility \\ Prepared by the European I Department \\ (In consultation with other departments) \\ Approved by Carlo Cottarelli and G. Russell Kincaid \\ June 17,2003
}

Contents

Page

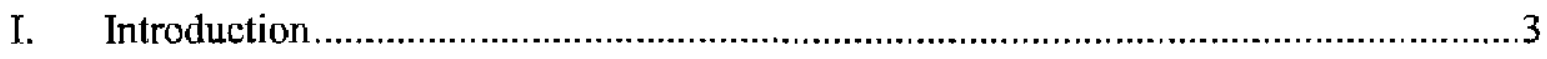

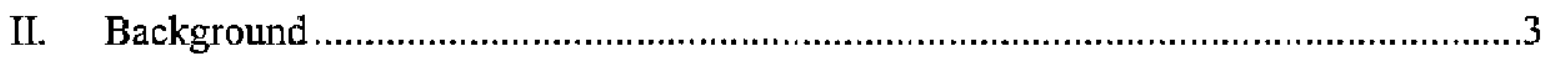

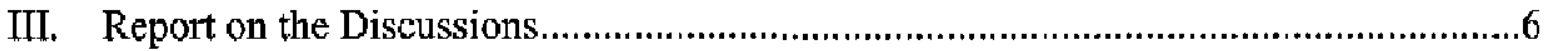

A. Macroeconomic Outlook ..............................................................................6

B. The Policy Implementation Framework and Governance .................................

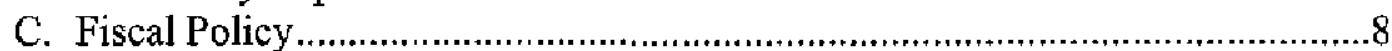

D. Monetary and Exchange Rate Policy .....................................................10

E. Structural Reform.....................................................................................10

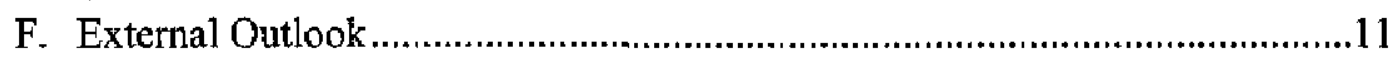

G. Program Monitoring...............................................................................13

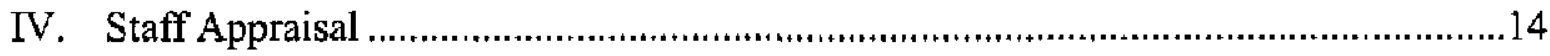

Text Box

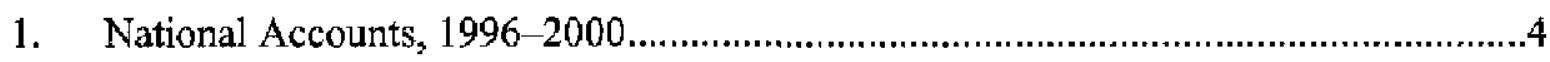

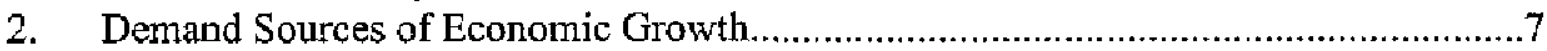

3. Structural Conditionality...............................................................................

Figures

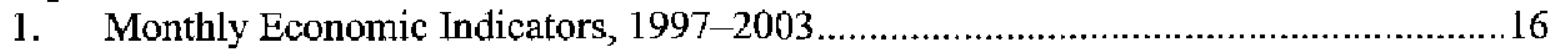

2. Economic Developments and Prospects, 1994-2003 .............................................17 
Tables

1. Performance Under the PRGF Artangement ....................................................18

2. Basic Indicators and Macroeconomic Framework, 1998-2006 ............................20

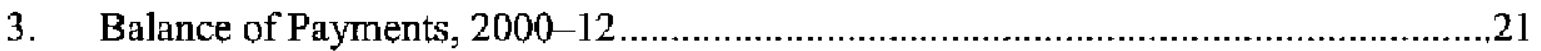

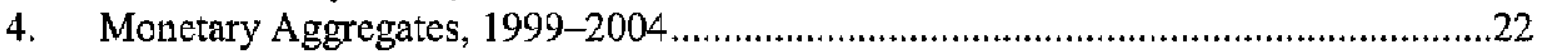

5. Government Revenues and Expenditures (In percent of GDP) 1999-2003 ............23

6. Government Revenues and Expenditures (In millions of Lek), 1999-2003 ,............24

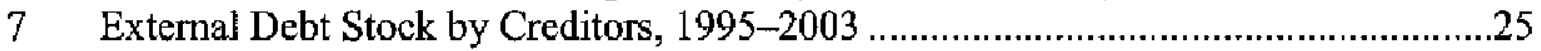

8. External Financing Requirement and Sources, 2000-06 .....................................26

9. Indicators of External and Financial Vulnerability, 1995-2003 _..........................27

10. Projected Payments to the Fund as of April 30, 2003.....................................28

11. Schedule of Purchases under PRGF Arrangement ............................................29

Appendices

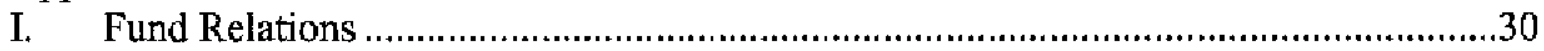

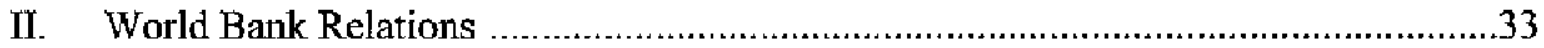

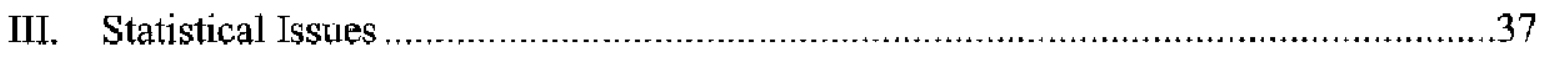

Attachments

I. Letter of Intent and Supplementary Memorandum on

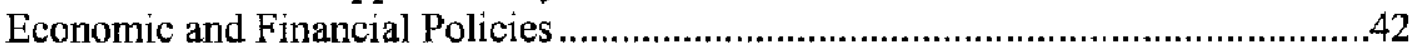

$\underline{\text { II. } \quad \text { Technical Memorandum of Understanding } \ldots \ldots \ldots \ldots \ldots \ldots \ldots \ldots \ldots \ldots \ldots \ldots \ldots \ldots \ldots \ldots \ldots}$ 


\section{INTRODUCTION}

1. A staff team ${ }^{1}$ visited Tirana during April 14-29, 2003 to conduct discussions for the second review under the PRGF arrangement-approved on June 21, 2002, consistent with the authorities' November 2001 National Strategy for Socio-Economic Development (NSSED) - and a financing assurances review. The first review under the arrangement and the 2002 Article IV Consultation were concluded on February 26, 2003. Executive Directors commended the authorities' sound macroeconomic management, but noted mixed policy implementation under the PRGF. The first NSSED Progress Report (PR) was issued in May 2003, and a Joint Staff Assessment (JSA) is circulated separately. In the attached Letter of Intent and the supplementary Memorandum on Economic and Financial Policies (MEFP), the Albanian authorities request completion of the second program review and financing assurances review. The MEFP expands on the discussion included in the PR on policies regarding tax and customs administrations, and updates the macroeconomic framework included in the latter document, in particular to incorporate the new national accounts.

2. Albania's economic program is also supported by the World Bank through several sectoral lending programs and a Poverty Reduction Support Credit (PRSC) approved in June 2002. In April 2003, FIAS presented a study of administrative barriers to investment.

3. Political stability remains weak and policy debate confrontational-a situation that is likely to continue, with local elections coming up in October and the ruling Socialist Party's leadership convention in December. These factors have adversely affected the pace of reform, notwithstanding the authorities' declared commitment to strengthen policy implementation and governance. Negotiations on a Stabilization and Association Agreement with the EU commenced in February 2003.

4. The coverage and quality of macroeconomic data remain weak, despite recent improvements. Albania participates in the GDDS. A ROSC on data dissemination was prepared in June 2000. A June 2002 safeguards assessment of the Bank of Albania (BoA) indicated that controls are broadly adequate, and the $\mathrm{BoA}$ has been addressing remaining minor weaknesses. A fiscal ROSC was finalized in June 2003, identifying weaknesses in the consistency and transparency of budgetary and tax regulations and procedures.

\section{BACKGROUND}

5. Performance under the program has remained mixed. Macroeconomic stability has been maintained and, up to end-March 2003, all quantitative performance criteria were met, but the indicative targets for revenue collection were missed. Although structural

\footnotetext{
' Messrs. Samiei (head), Martijn, and Ms. Vladkova-Hollar (all EU1), Ms. Tumbarello (PDR), Ms. Simard (FAD), and Mr. Olters ( resident representative). The team met with Prime Minister Nano, Finance Minister Islami, Bank of Albania Governor Cani, other officials, parliamentarians, and representatives from the banking and business communities.
} 
reforms have continued, further delays in meeting several benchmarks have occurred (Table 1),

\section{Largely due to adverse supply shocks, economic growth is estimated to have} decelerated in 2002, to less than 5 percent, from an average of around 7 percent in recent years (Table 2, and Figures 1 and 2). Discussions with businesses indicated that the slowdown stemmed mainly from the electricity crisis in the first part of the year and floods in the fall, against the background of political uncertainties and a weak investment climate. The limited activity data-including the BoA's business survey - suggest a pickup in activity and exports since mid 2002. National accounts data for 1996-2000 were released in January, indicating that, while trend real growth was broadly in line with previous estimates, the current level of nominal GDP is somewhat higher (Box 1).

\section{Box 1. Albania; National Accounts, 1996-2000}

The recently released official National Accounts have replaced staff and authorities' GDP estimates. The new GDP figures take into account the large share of the non-observed activity in total output-estimated at about a third of total GDP. The GDP estimates are likely to be revised in light of planned technical assistance over the next two years, as improvements are made in the core data and the methodology. The main weaknesses affecting current analysis are:

- There are no constant price estimates of the expenditure side items. The official GDP deflator diverges markedly from CPI inflation during the entire period, and the two are difficult to reconcile.

- The poor quality of the core data underlying the expenditure side estimates of private sector activity and the deflator problems described above produce an excessively volatile series for private consumption and a large statistical discrepancy between the expenditure and production side estimates of real growth (over 3 percenlage points in certain years).

Implications for Past Analysis

- Trend growth of real GDP (about 6 percent per annum) is broadly in line with previous estimates, while nominal GDP in 2001 is now $31 / 2$ percent bigher than previously estimated.

- Because of the higher growth of nominal GDP, the tax revenue to GDP ratio during 1998-01 is now estimated to have increased by 2 percentage points, about half of previous estimates.

Albania: Tax Revenue as a Share of GDP (in percent), 1998-2001

\begin{tabular}{lcccc}
\hline & $\mathbf{1 9 9 8}$ & $\mathbf{1 9 9 9}$ & $\mathbf{2 0 0 0}$ & $\mathbf{2 0 0 1}$ \\
\hline Current data & 16.9 & 17.1 & 18.9 & 19.0 \\
Data in Jamuary 2003 Staff Report & 15.6 & 16.5 & 19.1 & 19.7 \\
\hline
\end{tabular}

\section{The supply shocks are also reflected in a continued large trade deficit,} notwithstanding a fiscal contraction by 0.7 percent of GDP (Table 3 ). Net imports were accommodated, in large part, by continued strong worker remittances and official inflows, 
and, possibly, also through the use of liquid foreign assets. ${ }^{2}$ The 2002 current account deficit is now estimated at 9.1 percent of GDP, above earlier projections (see below). The lek has remained broadly stable in effective terms and against the euro since mid-2002-implying a sizable appreciation against the dollar. International reserves rose to US\$911 million (about $4 \frac{1}{2}$ months of imports) at end-April 2003, in part as a result of valuation gains.

8. Encouraged by abating inflationary pressures, monetary policy was eased in April 2003. Year-on-year CPI inflation fell from $4 \frac{1}{2}$ percent in October 2002 (corrected for statistical bias) to 2 percent by end-2002 and to 23/4 percent in April 2003, mainly reflecting the receding impact of earlier supply shocks (Table 4). With lower inflation and the ongoing return of deposits to the banking system following the bank run in early 2002 , the BoA lowered its repo rate by 50 basis points to 8 percent in April 2003, partly reversing the increases of early 2002 . The spread between the T-bills rates and the repo and deposit rates has remained large and volatile.

9. Notwithstanding considerable revenue shortfalls, the overall fiscal deficit (excluding grants) declined to less than 7 percent of GDP in 2002 (Tables 5 and 6). The shortfall relative to the-ambitious - budget target was 1.3 percent of GDP-significantly more than estimated at the time of the February Board meeting. This, combined with reduced privatization receipts (already incorporated in the interim budget revision) and lower foreignfinanced investments, was mirrored in sharply reduced capital expenditures. Delays in the use of Italian support for subsidized electricity imports further reduced overall spending. The end-year indicative target for domestic government borrowing was marginally exceeded (by about 0.1 percent of GDP). Public debt declined to 62 percent of GDP owing to debt forgiveness.

\section{Tax collection in the first quarter of 2003 again fell short of the indicative} program targets. The authorities have proceeded to implement the agreed measures for strengthening revenue collection, including the computerization programs in the tax and customs directorates. Nonetheless, based on information made available after the mission, the shortfall has been around 6 percent ( 1.2 percent of GDP on an annualized basis). Due to lower expenditures, the overall deficit, however, remained well below the budget and the end-March performance criterion on domestic borrowing was met.

11. Some progress has been made in relation to privatization, electricity sector reform, and improving the business environment.

- The government is executing a limited restructuring of the Savings Bank prior to resuming marketing efforts in the fall. A pre-privatization of Albtelekom was initiated in February 2003, with IFC and EBRD participation. The company has started

${ }^{2}$ Foreign currency deposits-unlike lek deposits- have not fully recovered from the early 2002 bank run, suggesting that they may have been used for financing imports. 
repaying its domestic debts and most of its foreign financial claims bave been settled, but an end-March benchmark for settling all claims was missed.

- In the energy sector, the action plan targets for reducing losses and improving fee collection by the electricity company, KESH, were met during $2002 \mathrm{Q} 4$ and $2003 \mathrm{Q} 1$ - following slippage in the summer and fall of 2002. Meeting these targets is a condition for continued donor support. Owing to ample inflows into the main hydropower lake since the fall of 2002, power shortages have been limited and KESH's financial position has improved with the reduced import needs.

- The business climate has benefited from a recent revision of the instructions on forced tax collection and improvements in customs valuation - which in the past had been major sources of complaints.

- Free trade agreements with Macedonia, FYR, and Romania have recently been ratified, and those with Serbia and Montenegro, and Croatia are expected to follow by mid-2003.

\section{REPORT ON THE DISCUSSIONS}

12. The mission assessed the need for improvements in policy implementation and adjustments to the policy program. The assessment, which was informed by the information contained in the preliminary draft of the $\mathrm{PR}$, centered on:

- mixed program implementation so far, raising questions about the authorities" commitment to the planned structural reforms;

- the urgent need for improving governance and fighting corruption in order to strengthen the business climate, as underscored by FIAS, and to improve budgetary and tax procedures, as noted in the recent fiscal ROSC;

- the continuing revenue shortfalls;

- the impact of the adverse supply shocks in 2002 on growth and net exports; and

- the easing of inflationary pressures, calling for a re-evaluation of the monetary stance.

\section{A. Macroeconomic Outlook}

13. In 2003, growth is expected to return to its medium-term trend of 6 percent, as the effect of adverse shocks tapers off, and reform efforts are strengthened. Industrial activity recovered sharply during the second half of 2002, owing to improvement in electricity provision, and agricultural growth is expected to return to trend with the repair of the damage to irrigation systems due to the flooding. Public investment is also expected to contribute to higher growth (Box 2). The authorities also emphasized the beneficial effects of recent improvements in the business climate, which were recognized by representatives of 
the business community. Nonetheless, the mission argued that growth prospects remained subject to important downside risks, including threats to political stability, uncertain electricity supply, sluggish partner country growth, and slow implementation of structural reforms.

\section{Box 2. Albania: Demand Sources of Economic Growth}

Albania's growth performance has been stable and rapid since 1998, although its sources on the demand side have shown large variations. From 2001 onwards, private consumption has provided the largest contribution, in part driven by strong private transfers, especially remittances. However, recorded private consumption has been extremely volatile due to measurement problems and liquidity constraints. Public consumption has been more stable, contributing, on average, $1 / 2$ percentage point to growth.

\begin{tabular}{|c|c|c|c|c|c|c|c|}
\hline & 1997 & 1998 & 1999 & 2000 & 2001 & $\begin{array}{r}2002 \\
\text { Est. }\end{array}$ & $\begin{array}{l}2003 \\
\text { Proj. }\end{array}$ \\
\hline Real GDP growth $1 /$ & -10.5 & 12.1 & 83 & 7,7 & 6.8 & 4.7 & 6.0 \\
\hline Consumption & -12.6 & 13.3 & 1.1 & -1.1 & 6.9 & 8.1 & 4.6 \\
\hline Private & -12.1 & 12.8 & 0.5 & .1 .4 & 5.5 & 8.4 & 4.1 \\
\hline Public & $-0,5$ & 0,5 & 0.6 & 0.3 & 1.3 & -0.3 & 0.5 \\
\hline Irvestment & -3.8 & 1.0 & 5.1 & 5.1 & 4.2 & 0.6 & 1,9 \\
\hline Private & & -1.4 & 4.1 & 4.1 & 3.0 & 0.7 & 1.5 \\
\hline Public & & 2.4 & 1.1 & 1.0 & 1.2 & -0.1 & 0.4 \\
\hline Net exports & 2.4 & -5.1 & 1.5 & -80 & -3.9 & -3.5 & -0.3 \\
\hline Change in Inventiortes and Statistical Discrepancy & 3.5 & 2.8 & 0.6 & 11.7 & -0.4 & -0.5 & -0.3 \\
\hline
\end{tabular}

The slower economic growth in 2002 (to 4.7 percent), was largely driven by the decline in public and private investment. In addition, supply side shocks (i.e., a flood and the energy crisis) reduced export capacity, and the net export component of GDP growth was negative.

The modest expected recovery of GDP growth in 2003 is based on higher private investment and net exports. The projected improvement in the current account (already evident in first quarter data) is consistent with an improvement of the supply side of the economy and, therefore, with higher GDP growth. In particular the energy situation seems to be more favorable. On the demand side, the improvement of the current account is consistent with the expected fiscal consolidation, with a decline in the deficit by 0.5 percent of GDP.

\section{Building on the recently published national accounts, the authorities are} improving the economic database. Staff emphasized that such improvements are critical, as the lack of timely activity data and comprehensive balance of payments data, in particular, hamper policy evaluation and formulation. The MEFP was updated to reflect the planned steps, which focus on gathering better source data, with technical assistance provided by STA (MEFP, $q 23)$.

\section{B. The Policy Implementation Framework and Governance}

\section{The authorities have improved the policy implementation framework since the} October 2002 mission and reiterated their commitment to the program. Following last year's political and administrative changes, policy coordination and communication among ministries and agencies have improved, and progress has been made in the dialogue with the business community. The mission acknowledged these improvements as well as the 
acceleration in the implementation of reforms during 2003, but also noted that, again, several benchmarks and the indicative revenue targets had been missed.

16. The program was given a stronger focus on strengthening governance and the integrity and competence of public institutions. Recent surveys, in particular by FIAS, have indicated severe corruption problems and lack of professionalism throughout the public administration, undermining the business climate and taxpayer morale, and creating serious obstacles to investment and economic growth. The government has also been criticized for its appointment policies, which have affected confidence in public institutions. Progress in improving governance is a declared government priority, and the mission encouraged the authorities to back this general commitment by concrete measures, consistent with World Bank and EU advice. Accordingly, critical actions concerning proper hiring procedures and asset declaration for public officials were included as benchmarks under the program (MEFP, I18; PR 1104), which also features a self-assessment of anti-money Jaundering legislation. Regarding tax collection, recommendations of the fiscal ROSC for reducing tax officials' discretion by strengthening the transparency and consistency of procedures and regulations, were also included (MEFP, ๆ9; PR |86). However, the authorities pointed to the limits of regulatory measures in addressing corruption.

\section{Fiscal Policy}

\section{Recent performance has highlighted renewed risks to the revenue strategy.} Budgeted increases in tax revenue in 2003 were based on tax policy and administration measures. The authorities explained that the implementation of these measures had been broadly on track; and the program of measures for 2003 and beyond was updated (see below). They remained moderately confident that the revenue targets for 2003 budget as a whole could be met, in spite of the recent shortfalls. However, staff consider achieving the budgeted revenues to be unlikely, even though the $2003 \mathrm{Q} 1$ shortfalls were in part due to temporary factors. The main concerns and risks are:

- The worse-than-expected 2002 revenue performance and the further shortfalls in the first quarter of 2003 (based on data made available after the mission) have rendered the 2003 targets harder to achieve. ${ }^{3}$ The increase in budgeted revenues relative to the realization in 2002 is about $1 \frac{3 / 4}{4}$ percent of GDP, well above the estimate of 1 percent of GDP at the time of the February Board meeting.

\footnotetext{
${ }^{3}$ However, the interpretation of the first quarter shortfalls is complicated, as the tax and customs departments have followed internally-agreed targets that apportion a smaller share (relative to the program) of the annual total to the first quarter. The mission urged the authorities to ensure the consistency of internal and program targets. Nonetheless, given the practice of tailoring collection efforts to the internal targets, this deviation can explain at least part of the shortfall.
} 
- Several key measures for enhancing collection in 2003 have yet to become effective-in particular, the new excise tax law (to be enforced in July) and the gradual transfer of collection responsibilities for social security premiums to the tax department (starting in September 2003).

- Finally, tax projections are based on 6 percent GDP growth, which is subject to downside risks, and hard to assess, given the lack of reliable short-term activity data.

18. In view of these risks, the authorities are following staff advice to prepare, as a prior action for the second review, clear contingency plans for the remainder of the year, taking into account the budgeted overall deficit of $6 \frac{1}{2}$ percent of GDP (MEFP, 77 ). Experience from previous years indicates that while revenue shortfalls need not endanger the deficit ceilings, they could result in indiscriminate spending cuts. In 2002-due to these cuts, shortfalls in foreign financed projects, and capacity constraints (related mostly to fiscal decentralization) - spending on health care fell from 2.3 percent of GDP in 2001 to 2.1 percent, and on education from 3.3 percent of GDP to 2.9 percent. In 2003 , expenditures on poverty alleviation is budgeted to increase by 14 percent in real terms ( 1 percent of GDP). The proposed contingency plans attempt to shield these priority areas (at the expense of, in particular, electricity subsidies and operations and maintenance) and will be implemented depending on end-June revenue performance.

19. In addition, the revised MEFP includes measures to strengthen tax compliance In the reminder of 2003. These include implementing new computer administration systems for profit taxes and social insurance contributions, as well as the ASYCUDA system for two main customs offices, raising the number of registered taxpayers by over 10 percent, and strengthening audit and control procedures (MEFP, ๆ9; PR ๆ85).

20. Regarding 2004, the mission and the authorities preliminarily agreed on a further reduction in the overall deficit (excluding grants) to 6 percent of GDP, in line with the January 2003 staff report. Domestic borrowing is projected to decline to $2 \frac{1}{2}$ percent of GDP-1/4 percentage point higher than earlier projections, offsetting lower foreign financing of projects. Additional declines in identified foreign-financed projects could give rise to a further reassessment of the scope for increasing domestic borrowing in preparing the 2004 budget, while maintaining the overall deficit of 6 percent. While this increase would entail somewhat higher interest costs, it would help provide resources for priorities under the NSSED, without posing a threat to continued macroeconomic stability. Recent collection performance suggests that the current revenue projections for 2004-with a further increase of $1 / 2$ percent of GDP — could also require downward revision. Limiting the ensuing risks to planned infrastructure and pro-poor expenditures, and thereby growth and poverty alleviation objectives, will require effective prioritization and efforts to address capacity constraint in executing expenditure plans. Staff and the authorities will discuss the broad parameters of the 2004 budget and the updated MTEF in July.

21. The fiscal measures in the revised MEFP put more emphasis on improving governance, prompted by the fiscal ROSC and the FIAS report. In addition to addressing 
inconsistencies in tax law and regulations (mentioned above), the mission also emphasized the need for more transparent and stringent budget procedures-facilitating scrutiny by parliament and the public. The authorities will adopt initial improvements in the context of the 2004 budget, in particular by bringing forward the budget preparation process (MEFP, 『12).

22. Regularizing inter-enterprise arrears is taking more time than anticipated. The authorities reported that the complex process of reconciling the amounts (an end-March program benchmark) would be completed during the second quarter. A revised timetable was determined for a subsequent comprehensive settlement and designing strategies for ensuring the avoidance of new arrears (with a September 2003 performance criterion; MEFP, I13). (As noted in the ISA, the PR does not describe government policies in this area.) These strategies should also facilitate tax collection, which is hampered by nonpayments by public enterprises. The authorities expressed a possible need for further technical assistance.

\section{Monetary and Exchange Rate Policy}

23. The authorities and the staff concurred on the appropriateness of lowering interest rates. The increase in policy rates from 7 percent to $8 \frac{1}{2}$ percent in 2002 - when inflation was mostly above its 2-4 percent target range-was instrumental in limiting inflationary pressures and risks. As monetary stability has been restored, scope has emerged for gradually undoing those increases. Lowering interest rates could invoke some depreciation pressure on the exchange rate, which given the large current account deficit, and provided that price stability is maintained, would not be unwelcome.

24. Monetary management is strengthened further, with increased reliance on open market policies, and will remain in line with the monetary program. Policy implementation is complicated by a monopolistic banking sector and extensive dollarization - both in terms of deposits and loans to the private sector. Consistent with recent advice by $M F D$ and $L E G$, the monetary authorities intend to rely on enhanced use of secondary market transactions with the banks for injecting liquidity rather than on direct credit to the government (MEFP, $\mid 14$ ). The program assumes that, with lower interest rates, the switch from cash holdings to lek deposits will come to a halt and the share of forcigncurrency deposits will increase-also as the smaller banks, which focus on foreign-currency services, expend their networks. The target for NIR of the BoA serves to ensure an end-year coverage of at least 26 percent of broad money.

\section{E. Structural Reform}

25. The authorities agreed to focus on removing barriers to investment and addressing corruption in promoting private sector activity. They have formed a task force to prepare actions, consistent with the FIAS report, aiming to correct arduous procedures and fight corruption (MEFP, \19). Initial steps seek to ensure regular VAT refunds to importers and improve the tax appeals process. However, the authorities are also continuing the creation of new agencies for promoting foreign investments, exports, and 
small firms. The mission stressed the importance of keeping these entities small and nonbureaucratic, and urged that a planned scheme for providing and guaranteeing credit to small enterprises should not distort market functioning or create scope for abuse.

26. Restructuring Albtelekom and the Savings Bank should belp improve their services and future marketability (MEFP, $ๆ 20$; PR $\ 122$ and $¥ 132$ ). The mission acknowledged the progress in settling Albtelekom's financial claims and encouraged efforts to minimize the delay in completing the process. Regarding the Savings Bank, the authorities hope to select a buyer before end-2003. The mission urged further efforts to streamline this dominant bank before its sale in order to bolster competition, while ensuring provision of basic banking services in remote areas following the bank's privatization. The authorities announced plans to develop a new marketing channel for treasury bills outside the banking system, which should help limit the sizable interest margin relative to deposit rates. The mission urged the authorities to proceed with caution in reviving the domant stock marketin view of financial sector fragilities and substandard accounting and auditing practices-and to complete a feasibility study first. The mission advised against moving the primary auction of treasury bills from the BoA to this institution-in view of risks to liquidity management and to household access to treasury bills (MEFP, 122 ). The authorities have requested a Financial Sector Assessment Program (FSAP) to help guide the further development of the financial sector.

\section{Staff encouraged the authorities to further strengthen electricity sector reform.}

They welcomed recent improvements in meeting the action plan targets, as well as plans to speed up to mid-2004 the installation of meters for all customers (MEFP, 121 ). Success in both areas will be key to the planned elimination of subsidized electricity imports by 2005 . The authorities expected that, with increased domestic hydropower supply in 2003, the required subsidy would remain below the budget-in spite of the delayed introduction of this year's price increase (from January to April). The authorities assured the mission that with revised procedures, such delays would be avoided in the future.

\section{F. External Outlook}

28. The mission noted that the current account path had deteriorated sígnificantly from that envisaged at the start of the program (see tabulation). As noted, the worse-thanexpected 2002 outcome largely reflected supply shocks. ${ }^{4}$ In addition, the deterioration in 2003-04, relative to earlier projections, is mainly due to more cautious assumptions regarding exports and imports. While these changes imply reduced vulnerabilities relative to earlier projections and do not translate into a higher debt ratio (in part reflecting the non-

\footnotetext{
${ }^{4}$ The 2001 outcome does not provide a proper benchmark, as an end-year surge in remittances, linked to the introduction of euro notes, lowered the current account deficit by about 2 percent of GDP.
} 
debt-creating nature of most current account financing), they still underscore the need for scrutiny of the external outlook.

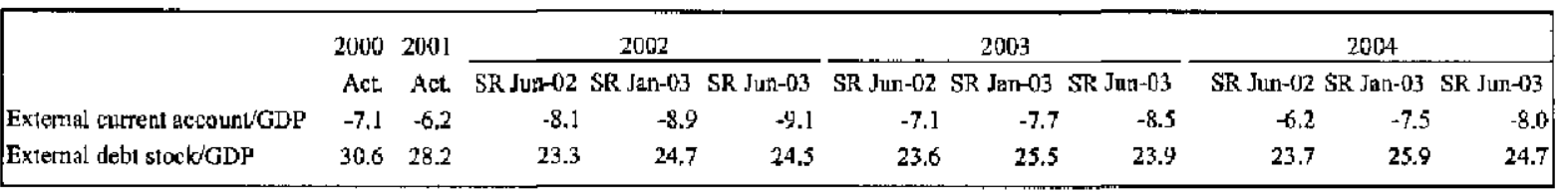

29. The projected improvement in the current account deficit, by almost 2 percent by 2006 , is consistent with the projected fiscal consolidation, and requires strong commitment to structural reform. In 2003 , the improved provision of electricity should help boost export performance and FDI, supporting the projected current account improvement by 0.6 percent of GDP. Over the medium term, strengthening the business environment and removing structural obstacles to exports and investment will be key to expanding the production of tradables. To this end, the authorities have prepared an export promotion strategy to be complemented with the steadfast implementation of wide-ranging policies to improve governance. However, in the staff's view, any further worsening in the current account relative the projected path, could also require adjustments in macroeconomic policies.

30. The external debt outlook is projected to remain manageable over the medium term (Table 7). Albania's external debt to GDP ratio is well below the average for PRGF countries, owing to favorable interest rates, high growth, and a gradual real appreciation." External debt service represented 6.4 percent of exports of goods and nonfactor services in 2002 , but is expected to increase to about 10 percent by 2010 before it starts to decline. ${ }^{6}$ This increase-which exceeds earlier projections, owing largely to the revised current account path-also reflects larger amortization payments starting in 2004 given the maturity profile of bilateral debt, rescheduling of outstanding arrears, ${ }^{7}$ and more cautious assumptions about future borrowing terms. Regarding nonconcessional debt ceilings under the program, the mission and the authorities agreed on an increase of US\$30 million (0.5 percent of GDP) in the end-2003 ceiling, relative to the outcome in 2002-which was US $\$ 60$ million below the

${ }^{5}$ The NPV of the external public debt amounted to 15.3 percent of GDP as of March 2003.

${ }^{6}$ As a ratio to central government revenues, it is projected to increase from 5 percent to 8 percent-still comfortable compared with an average of 20 percent for other PRGF countries.

${ }^{7}$ External arrears at end-May 2003 amounts to 2.3 percent of GDP. The current projections assume a restructuring of these arrears by end-2006, with a resulting increase in the debt service ratio of 0.5 percent of exports of goods and nonfactor services during 2006-2012. 
programmed ceiling. ${ }^{8}$ These loans will mainly finance road and hospital projects-with positive impacts on growth and poverty reduction. However, given the challenges described above, staff urged the authorities to make additional efforts to carefully appraise and prioritize the proposed projects and remain committed to prudent debt management (MEFP, ๆ24).

31. The program for 2003 is fully financed. The projected debt flow figures as well as the external outlook indicate that Albania will not have difficulty in meeting its current and future external debt service obligations to the Fund (Tables 8, 9, and 10).

32. The authorities remain committed to rescheduling the remaining external arrears with official creditors within the program period (MEFP, $\uparrow 25$ ), by pursuing a revised strategy, in cooperation with creditors. They have already successfully rescheduled arrears to Paris Club creditors, and delays in reaching agreement with non-Paris Club creditors is due to failed attempts to receive comparable terms. They plan to finalize restructuring agreements with the Czech Republic and the Slovak Republic. They will submit to parliament restructuring agreements already reached with Hungary (US\$32 million) for ratification by the end of 2003 (structural benchmark). Parliament approved in May 2003 a set of criteria for settling Albania's remaining arrears to official and commercial creditors. Moreover, the reconciliation on arrears in ruble denominated has been completed, with the exception of Bulgaria and Vietnam for very small amounts. However, an agreement on the exchange rate has been reached only with Hungary.

\section{G. Program Monitoring}

33. Conditionality under the program has been prepared for the period up to March 2004. The fourth disbursement under the program is scheduled to take place upon observance of the end-September 2003 performance criteria and completion of the third review scheduled for January 2004 (MEFP). Quantitative performance criteria are specified in the MEFP. The proposed structural conditionality focuses on measures critical to meeting the program objectives, is consistent with the NSSED and the PR, and has been set in close collaboration with the World Bank (Box 3 and MEFP).

\footnotetext{
${ }^{8}$ This adjustment raises the ratio of debt service to exports during 2009-2012 by 0.7 percentage point, cumulatively.
} 
Box 3. Albania: Structural Conditionality

\section{Coverage of Structural Conditionality in the Current Program}

Structural conditionality (MEFP, Table 3) has been expanded to include the new focus on improving governancc. All conditions are doemed critical to the goals of the program: fiscal sustainability and sustained private sector-led growth. Conditionality includes:

- Measures to strengthen governance and improve the business climate-including rules for public officials and civil servants (enhancing confidence in public institutions), and more transparent and consistent fiscal regulations and procedures.

- Tax and customs administration measures to underpin the revenue projections.

- Efforts to regularize domestic inter-enterprise and external arrcars, essential to manage contingent fiscal Liabilities.

- Actions to support the privatization process - key to improving crucial business services and providing resources for future budgets. The World Bank and the IFC are providing technical support.

Status of Previous Structural Conditionality and Relevant Measures

Performance against structural conditionality for the second review of the PRGF arrangement has been mixed (See Table 1).

\section{Structural Areas Covered by World Bank Lending and Conditionality}

The Bank's 2002-05 Country Assistance Strategy focuses on governance and institution building; sustainable private sector development; and human development. For 2003, the Bank is planning a second PRSC, and is continuing several sectoral lending projects-including in the energy and financial sectors (See Appendix II).

\section{STAFF APPRAISAL}

34. In the aftermath of adverse supply shocks, and with further measures to promote investment, growth of production and exports is expected to recover somewhat in 2003. This assessment is supported by recent exports and business survey data and some acceleration in structural reform, although overall program implementation bas remained mixed.

35. The urgency of improving governance, as a precondition for sustained growth, justifies inclusion of conditionality in this area. Widespread corruption and other governance problems are major obstacles to private sector activity and poverty reduction. Addressing these issues will require determined and persistent action in many areasimproving regulations, fighting abuse, and providing visible leadership. Program measures focus on promoting transparent and consistent fiscal regulations - recommended in the fiscal ROSC - and the competence and integrity of civil servants and public officials.

\section{Efforts to remove barriers to investment and ensure a reliable provision of} electricity are also critical to private-sector-led growth. The initiative for addressing bureaucratic bottlenecks based on the FIAS report provides a good framework for coordinated action. Both in this context and more generally, the authorities should draw on feedback provided by the business community and other stakeholders. 
37. While the fiscal balances have broadly remained within the program targets, both revenues and expenditures have been lower than projected. For 2003, staff's assessment of tax revenue performance is less optimistic than the authorities'. Weak revenue performance poses risks to the budget, necessitating well-prepared expenditure contingencies. For the medium term, given the still large demands on the budget for poverty reduction and infrastructural goals, this also underscores the importance of improved tax administration and prudence in assessing the revenue outlook, better expenditure prioritization and making contingency planning an integral part of the budgetary process (as stressed in the JSA), and avoiding further delays in the execution of foreign financed projects. As the current primary budget deficit is close to a debt-stabilizing level (under the projected interest rate and GDP growth), planned further consolidation should put the debt. ratio on a declining path.

38. With risks to inflation and monetary stability diminishing, policy interest rates should be eased gradually following the first step in April. Improving the functioning of financial markets requires better marketing of treasury bills and actions to address the dominance of the Savings Bank before its privatization.

39. External sustainability hinges on further improvements in the business climate, to expand the production of tradables, and ongoing fiscal consolidation. While the projected increase in debt service stays within prudent limits, it does raise vulnerability to further shocks-underscoring the need for fiscal prudence and structural reform. Financing future current account deficits with a relatively small share of debt creating inflows will crucially depend on the ability to attract foreign investors. Improvements in debt management and project evaluation should complement these efforts. The authorities are perusing good faith efforts to reach collaborative agreements with official and private creditors on the remaining (pre-1991) arrears, and should avoid further delays. The program, if appropriately implemented, would contribute to the maintenance of orderly relation between Albania and its creditors.

40. While the authorities remain committed to the program, serious risks to growth and the execution of the poverty reduction strategy remain. Exogenous factors include the vulnerability of power supply to weather conditions and of exports and private inflows to partner country growth. But internal factors pose the main risks: political uncertainties, resistance to efforts to improve governance, and limited implementation capacity. However, the program generally enjoys strong ownership, and the authorities reiterated their commitment to strengthen its implementation.

\section{Staff supports the authorities" request for completing the second program} review and the financing assurances review. The supplementary MEFP provides a solid basis for economic policies aimed at sustaining growth and preserving macroeconomic stability. 
Figure 1. Albania: Monthly Economic Indicators, 1997-2003
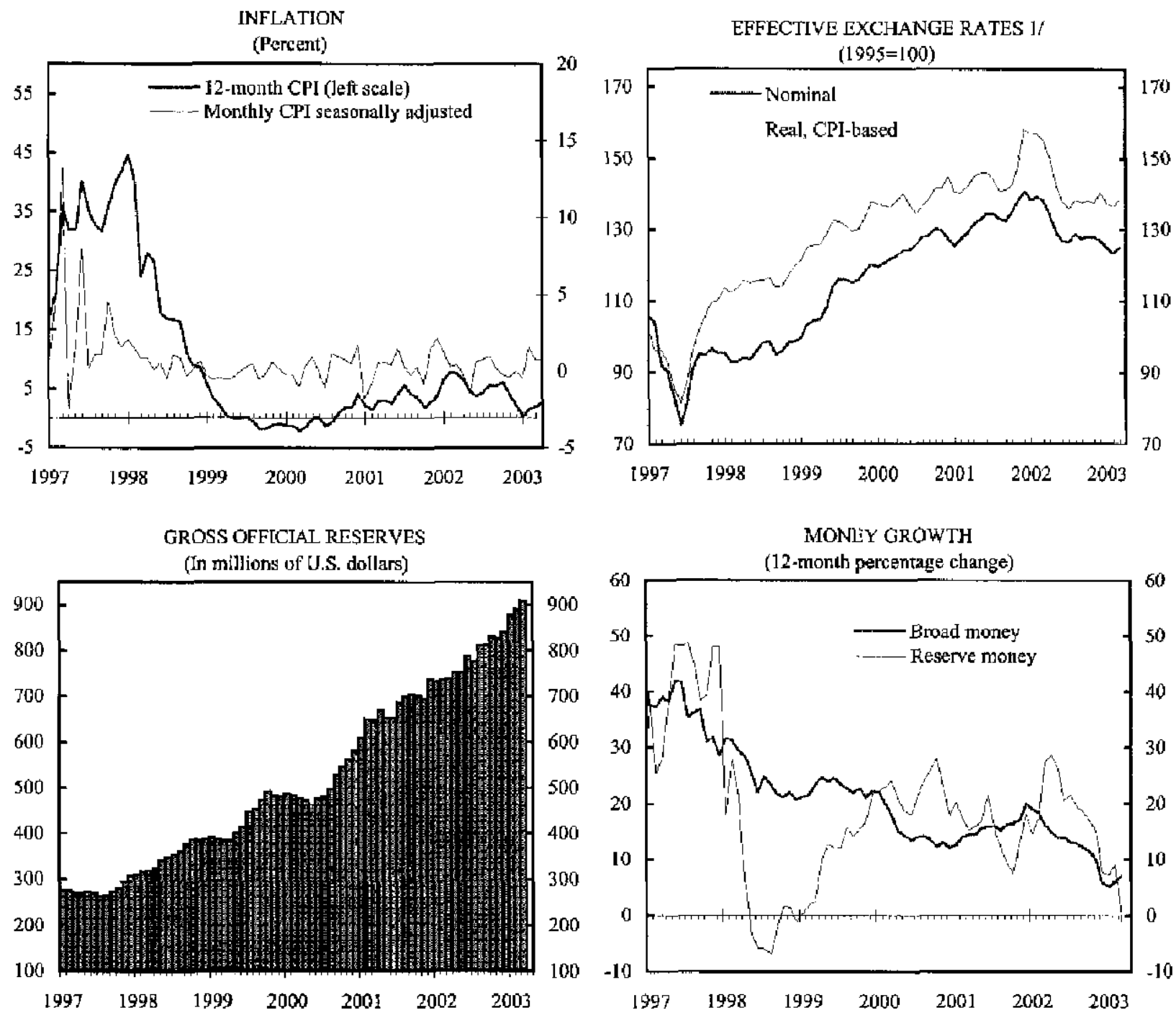

THREE-MONTH T-BILL RATE $2 /$

(Percent per year)

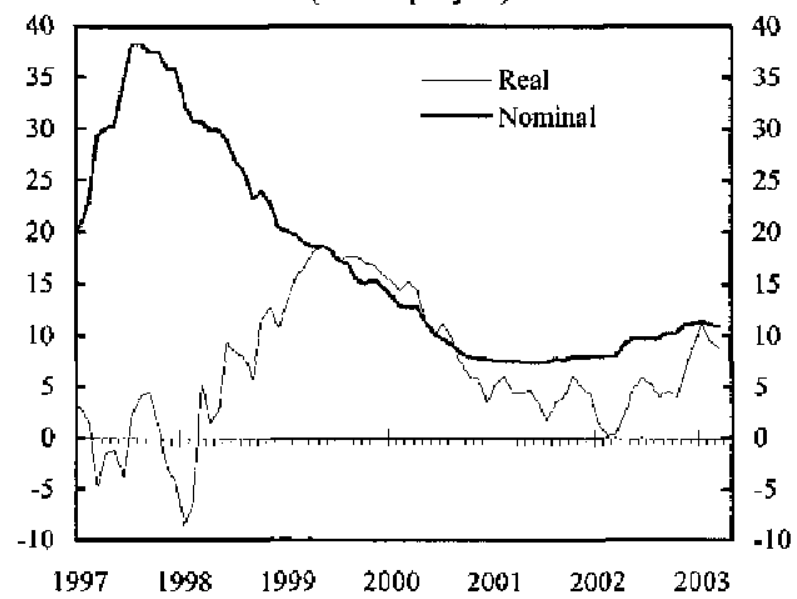

EXPORTS, IMPORTS, AND TRADE BALANCE

(In millions of eurs, seas. adj.)

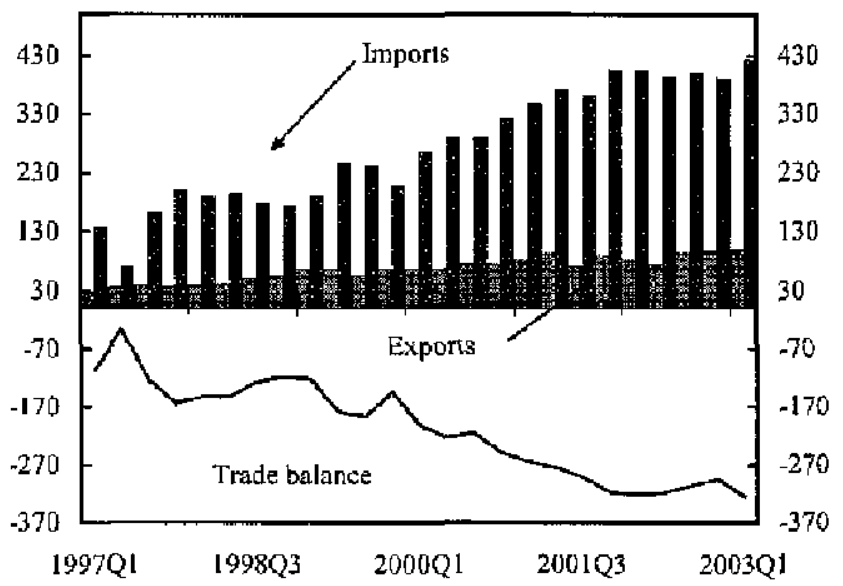

Sources: Bank of Albania, Ministry of Finance, INSTAT, and Fund staff estimates.

1/ Against the currencies of Albania's major trading partners. A rise in the graph indicates appreciation.

2) The real rate using annualized seasonally adjusted CPI inflation. 
Figure 2. Albania: Economic Developments and Prospects, 1994-2003 1/
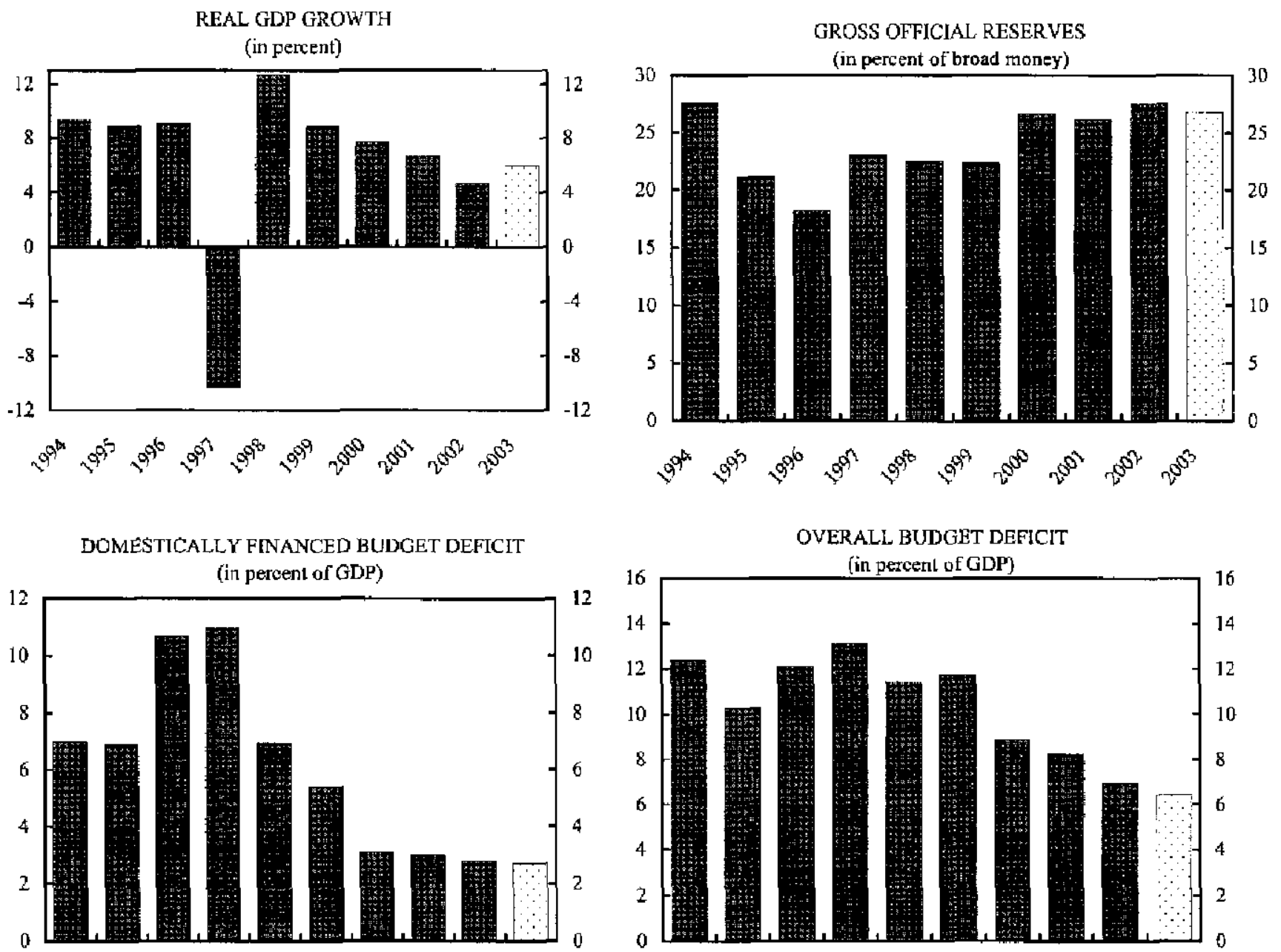

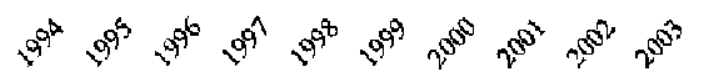

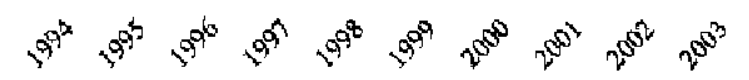

CURRENT ACCOUNT DEFICIT
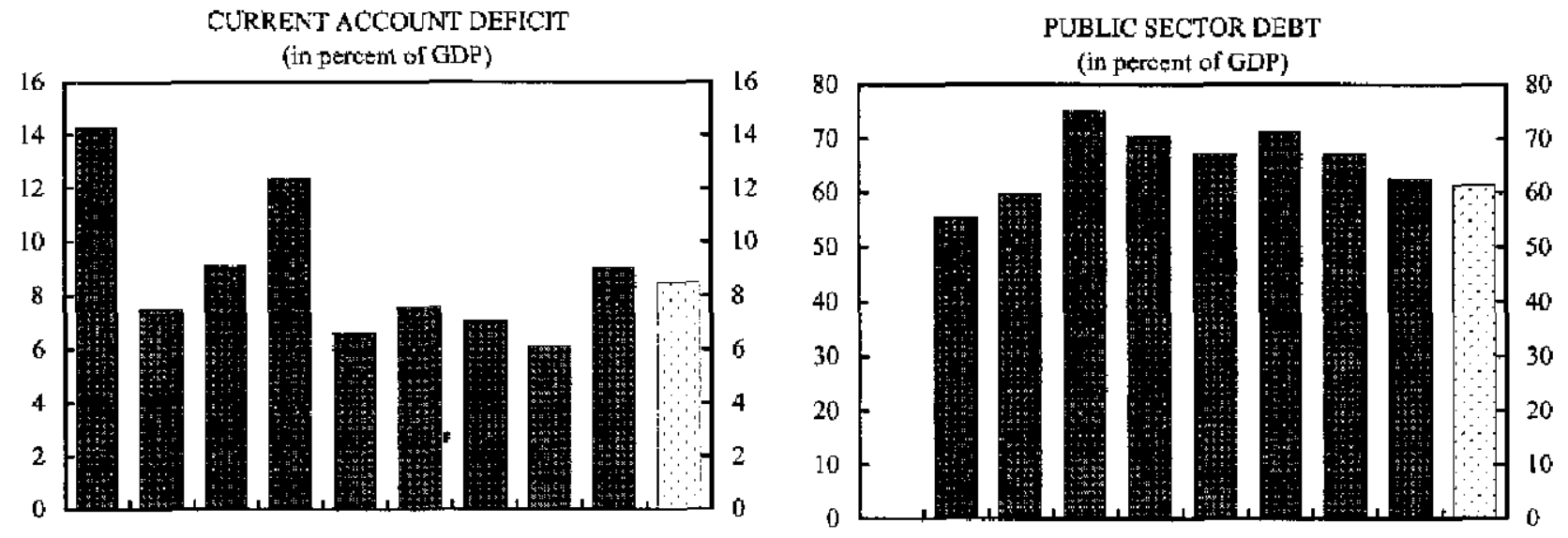

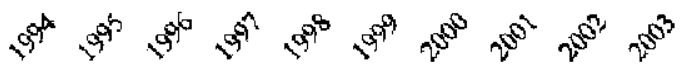

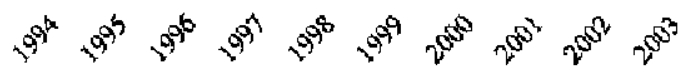

Source: Albanian authorities and Fund staff estimates.

I/ GDP numbers for 2001 and 2002 are estimates. 
Table 1. Albania: Performance Under the PRGF Arrangement

Measures

A. Prior Actinas under Initlal Program

1. Meel the largets to end-April 2002, on a cumulative basis, consistent with the two-year action plan for the electricity sector reform (MEFP, \{27; and NSSED, Section IV.F.3).

2. Prepare the first comprehensive quarterly report updating the stock of extemal arrears as of end-

March 2002, and identifying progess in, and plans for the cleamence of external arrears (MEFP, \{32).

3. Finalize domestic process for the external arears rescheduling agreement with Rossia and pay the already scheduled amounts (interest and principle) (MEFP, $\{32$ ).

4. Parlianacnt to approve mendments to the 2002 budget consistent with the revised Memorandutt of Economic and Findncial Poljcies (MEFF, ๆ13).

\section{B. Performance Criteria under Initial Program}

1. Parlianent to pass legislation to close down duty-fiee shops at all land cros sing points in border areas to reduce scope for struggling and fiscal evasion (MEFP, ๆ16).

C. Structural Benchmarks under Initial Program

1. Resolve all of Albtelekom's financiel and legal disputes in preparing it for privatization (MEFP, 26; and NSSED, Section NV.F.4)

2. Internal Andit Lnit to produce quarterly reports on the functioning of customs gystem, including specific recommendatione for the remedy of identified shortcomings, snd Director-General to send reports on corrective measures taken by the Customs Department

3. The Tar Department ta prepare quarterly reperts on the controls of the use of cash registers and penalties impo sed on misusers(MEFP, $\uparrow 16$; and NSSED, Section IV.C).

4. Draft. and implement as action plan jointly prepared by Tax and Customs Departments to redace fiscal evasion through underinvoicing (MEFP, $\| 16$; and NSSED, Section [V.C).

5. Bxtend the tax base through (MEFP, 17 )

a) introducing environmental taxes (by September 1,2002 ) and a tax on the ownexship of agriculitural land (by October 1,2002 ) and

b) increasing the number of registered taxpayers from 47,000 at end-2001 to 57,000 at end-2002 (NSSED, Set:lion TV.C).

6. With regard to arrears within the public seetor (MEFP, 919 ):

a) finalize the recording of inter-enterprise arrears and tax arrears, complete the reconciliation process, and sign bilateral memoranda of understanding indicating the amouth of net overdne payables/receivables as of Decunber 31,2001 ; and

b) determine the amount of inter-entenprise arears that can be cancelled through multilateral nettings.

7. Select a buyer for the Saving Bank and initiate negotiations (MEFP, 126 ; and NSSED, Section YV.D.2).

8. Finalize a major study of the energy sector with the aim of deternining electricity tariffs based on long-ran unarginal costs and phasing out subsidies by end-2004 (MEFP, "a27; and NSSED, Section

IV.F.3),

9. Prepare quarterly reports (within one month of the end of each quarter), identifying progress in and plans for the clearance of external arrears, and updating the stock of extemal arrears (MEFP, 132).

10. In relation to monetary policy and the financial sector (MEFP, 128; and NSSED, Section TV.D.2): a) introduce govermment securities wilh a maturity of at leasl two yexrs;

b) promote the sale of T-bills to the public through Albapost and further improve the aceessibility of the BoA's window for selling T-bills.

\section{Deadline Current Status}

End-September 2002

End-September 2002

Throughoul

Throughout

Bnd-Jine 2002

End-October 2002

End-October 2002

End-June 2002

End-September 2002

Throughout

End-June 2002

Throughout
Completer

Completed

Completed

Completed

Compteted as a Prior Action for First Review

Converted to Strustural Benchmark for Second Review

In observance

ln observance

In observance

Delnyed to January 2003

Missed

Delayed to June 2003

Converted to Structural Benchmark for Second Review

Not met due to lack of interested buyers

Completed in Jaruary 2003

In observance

Completed end-0ctober 2002

Dropped; BoA provided substitute venue for sale of $\mathrm{T}$ bills 
Table 1. Albania: Performance Under the PRGF Arrangement

\section{Measures}

Deadline

Current Status

D. Prior Actions for the First Review

1. Parliament to approve the 2003 budget consistent with this Supplementary Menuoratioum of Financial and Economic Policies (MEFP, 18).

2. Pirliament to pass legislation to close daty-free shops at all land crossings in border areas to reduce scope for smuggling and tax evasion (MEFP, †9).

Completed

\section{E. Structural Benchmarks for the Seco od Review}

1. To strengthen tax and customs adminis tration: recruit around 40 additional appropriately skilled and experienced staff in the Tax Degartment's headquarters (MEFP, 110 ); revise procedures and manuals for the enfored collection of taxes (MEFP, $\lceil 11$ ); and establish a post-clearance control unit with a staff of up to 10 senior inspectors, concentrating on vahation, tiassification, origin, excmption, and suspenston clains, whils updaling reference prices regularly (MEFP, $\$ 10)$.

2. Detennine the amount of domestic arrears within the public sector that can be cancelled through govempent-assisted multilateral repayments and design strategies for promoting the full and tirnely payment of all obligations conlrasted thereafler (MEFP, 914 ).

3. To promete the privatization process: complete the transfer of pension functions from Savings Bank to Albrpost (MEFP, \$19); and settle the remaining foncial clains of Albtelekor (MEFP, II ).

End-May 2003

Elid-March 2003

Eud-Manch 2003 arrears and progress in clearing these arrenrs (MEFP, 125 ).
Completed, except for establishment of post-clearance conlrol unit, which has a new test-date of end-July 2003

Converted to a Perfornance Criterion with test-date endSeptember 2003

Pensiun furnctions have bers trassferred. Settling remaining financial claims of Albte]ekom has been converted to a Structural Benclumark for the Third Review with test-date end-Sspteraber 2003

In observance, as of March 2003 
Table 2. Albania: Basic Indicators and Macroeconounic Framework, 1998006

\begin{tabular}{|c|c|c|c|c|c|c|c|c|c|c|c|c|}
\hline & \multirow[t]{2}{*}{1998} & \multirow[t]{2}{*}{1999} & \multirow[t]{2}{*}{2000} & \multirow[t]{2}{*}{2001} & \multicolumn{2}{|c|}{2002} & \multicolumn{2}{|c|}{2003} & \multicolumn{2}{|c|}{2004} & 20105 & \multirow{2}{*}{$\frac{2006}{\text { Pruj. }}$} \\
\hline & & & & & $5 \mathrm{KR}(\mathrm{ith}$ & Est. & SR0L:03 & Proj & SIROI:03 & Proj. & Proj. & \\
\hline & \multicolumn{12}{|c|}{ [Percent change) } \\
\hline Real GDP |f & 12.7 & 8.9 & 7.7 & 6.8 & 4.7 & 4.7 & 6.0 & 6,0 & 6.0 & 8.0 & 6.0 & 6.0 \\
\hline Retai] pricts [avg.) & 20.9 & {$[n, 4$} & 00 & 3.1 & 5.6 & 5.4 & 2.0 & 3.0 & 3.0 & 3.0 & 3.0 & 9.1) \\
\hline \multirow[t]{2}{*}{ Retn il prices (end-pericd) } & 8.7 & -1.0 & 4.2 & 3.5 & 3.0 & $\underline{2}-1$ & 3.11 & 3.6 & $2 n$ & 3.0 & 3.0 & $3.1)$ \\
\hline & \multicolumn{12}{|c|}{ (In percent of GDP) } \\
\hline \multicolumn{13}{|l|}{ Savijg-investmelut balance } \\
\hline Forriglu saving 2 & 6.9 & 7.7 & 71 & 6.2 & 8.9 & 5.. 1 & 3.7 & 8.5 & 7.0 & 8.i & 7.4 & 6.9 \\
\hline Dulueslie saving & 6.9 & 9.1 & 13.0 & 16.4 & 10.1 & 12.7 & เา.8 & 14.0 & $\{3.9$ & 15.3 & 160 & 16.6 \\
\hline Pubjic 3/ & -5.8 & -5.8 & -2.5 & -1.2 & -1.0 & -0.3 & 0.1 & 0.2 & 2.3 & 1.0 & 1.2 & 1,4 \\
\hline Private & 12.8 & 14.9 & 15.5 & 17.6 & {$[1.1$} & 13.1 & 6.7 & 13.8 & 11.6 & 14.9 & 14.6 & 15.2 \\
\hline Irwestment & 13.6 & 16.7 & 200 & 22.5 & 19,0 & 21.8 & 19.5 & 22.6 & 21.5 & 23.3 & 2.3 .4 & 23.5 \\
\hline Pulstic & 5.6 & 5.9 & 6.4 & 7.00 & 6.5 & 6.6 & 0.7 & 6.7 & 8.3 & 7.0 & 7.0 & 7.0 \\
\hline Priwate & 8.0 & 10.8 & 13.7 & 15.5 & 12.5 & 15.2 & 12.6 & 15.9 & 13.2 & 16,3 & 10.4 & 16.5 \\
\hline \multicolumn{13}{|l|}{ Piscal senter } \\
\hline Revenus and grants $4 /$ & 23.9 & 24.3 & 22.9 & 22.8 & 24.1 & 22.7 & 25.1 & 23.9 & 26.2 & 25.0 & 2.5 .3 & 25.3 \\
\hline Tax revenue & 16.9 & 17.1 & 18.9 & 19.0 & 20.4 & 19.0 & 21.4 & 201.2 & 22.2 & 21.2 & 21.6 & 21.6 \\
\hline Expendituras & 39.2 & 33.9 & 30.7 & $31) .4$ & 31.0 & 29.0 & 31.0 & 29.6 & 31.3 & 30.2 & 30.4 & 30.1 \\
\hline Primary & 24.8 & 26.6 & 25.2 & 26.2 & 27.2 & 25.4 & 27.2 & 26.0 & 27.3 & 26.4 & 26.7 & 26.4 \\
\hline Tnterest $\$$ & ก.ร & 7.2 & 5.6 & 4.2 & 3.7 & 3.7 & 3.8 & 3.6 & 4.0 & 3.5 & 3.8 & 3.8 \\
\hline Overall balanter (exeluding grants) & .114 & .118 & .8 .9 & .8 .2 & -7.5 & -6.9 & .6 .6 & - 4 & -6.0 & -6.0 & -5.8 & -5.6 \\
\hline 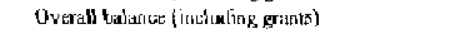 & -9.7 & -9.1 & .7 .9 & -7.6 & -6.9 & -6.3 & $-6,0$ & -5.8 & -5.2 & $-5: 2$ & -5.1 & -5.6 \\
\hline ITinzary balance (including gralnt5) & $-] .2$ & -1.9 & -2.3 & -3.4 & -3.2 & -2.6 & -22 & -2.2 & $\cdot 1.2$ & -1.4 & -1.3 & -1.2 \\
\hline Inomestic borrawing & 6.9 & 5.4 & 3.1 & 3.0 & 3.0 & 3.2 & 2.8 & 2.7 & 2.1 & 2.4 & 2.0 & 1.8 \\
\hline Frivalizating receipts & 0.0 & 0.2 & 1.6 & 2.1 & 0.2 & 0.1 & 0.1 & 0.1 & 0.4 & 0.3 & 0.3 & 0.3 \\
\hline laoreign finares & 2.8 & 3.6 & 3.2 & 2.6 & 3.8 & 3.0 & th. 1 & 2.9 & 2.6 & 2.4 & 2.4 & 2.9 \\
\hline Putlic Detrt & 70.9 & 67.5 & 71.6 & 67.6 & 64.4 & 62.9 & 64.6 & 61.8 & 63.7 & 61.7 & 59.8 & 58.2 \\
\hline Domestic $6 \%$ & 35. 1 & 36.3 & 41.0 & 39.4 & 39.6 & 38.4 & 39.1 & 37.9 & 37.8 & 37.0 & 35.7 & 34.3 \\
\hline Fxier|ast (including publicly guarantecd) $\%$ & 35.8 & 31.2 & 30.6 & 28.2 & 24.7 & 24.5 & 25.5 & 23.9 & 25.9 & 347 & 24.1 & 23.9 \\
\hline \multicolumn{13}{|l|}{ Monctary iroticautus } \\
\hline Btogad I unлty growth (in percent) & 20.6 & 22.3 & 12.0 & 20.2 & 7.6 & 5.7 & 8.9 & 8.5 & 8.2 & 9.2 & 9.2 & 9.2 \\
\hline Prinutc ercdil srouth (in perceltt) & 14.7 & 29.4 & 26.9 & 43.1 & ] 3,0 & 32.1 & 26.5 & 28.0 & $\ldots$ & 27.1 & $\ldots$ & $\ldots$ \\
\hline Velocity & 1.9 & 1.7 & 1.6 & 1.6 & 1.6 & 1.6 & 1.6 & 1.6 & 1.6 & 1.6 & 1.6 & 1.6 \\
\hline \multirow[t]{2}{*}{ 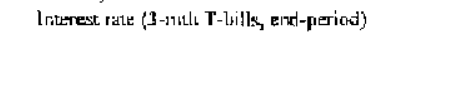 } & 20.4 & 14.8 & 7.8 & 8.0 & $\cdots$ & 11.2 & $\ldots$ & $\cdots$ & $\ldots$ & $\cdots$ & $\cdots$ & $\cdots$ \\
\hline & \multicolumn{12}{|c|}{ (In millious of U. S. dullars) } \\
\hline Cxterizal anctor & & & & & & & & & & & & \\
\hline Trade bulunce 8 & $.62 i$ & -663 & -821 & -1027 & $-\operatorname{l9g6}$ & -1155 & .1129 & -1292 & -1355 & -1.375 & -1451 & -1541 \\
\hline (in percent ut GitJP) & -22.1 & -18.7 & .21 .4 & .24 .1 & -23.7 & -23.9 & -22.8 & .23 .3 & -22.9 & -22.9 & -220 & -21.3 \\
\hline Cimrelt account balance & .195 & -272 & -274 & -263 & -410 & -440 & +381 & -471 & .443 & -482 & $-4,97$ & +498 \\
\hline (in percent of GDP) & -6.9 & .7 .7 & -7.1 & -6.2 & -8.9 & -9.1 & -7.7 & -8.5 & .7 .6 & -8.0 & -7.4 & -6.9 \\
\hline (in percent of GEP; ikt. wTitizl Iran ifers) & $-3,5$ & -3.7 & -4.2 & -3.2 & -6.4 & -6.6 & -5.3 & -6.1 & -5.5 & -3.7 & -5.1 & -5.1 \\
\hline Qfticial crarsfiers & 89 & 139 & $\mathrm{ll1}$ & 126 & IIS & 121 & 119 & 134 & 120 & 1.38 & 135 & 132 \\
\hline (in percent uf CinP) & 3.2 & 3.9 & 2.9 & 3.0 & 2.3 & 2.5 & 2.4 & 2.4 & 2.1 & 2.3 & 2.0 & 1.8 \\
\hline Oross isternational rescrucs & 384 & 48.5 & $60 \%$ & 754 & 800 & 860 & 819 & 898 & 9003 & 9,32 & 943 & 952 \\
\hline (im months of ilmports of goods and services) & 3.7 & 3.8 & 4.1 & 43 & 4.9 & 4.5 & 4.6 & 4.4 & 4.3 & 4,3 & 4.1 & 3.8 \\
\hline (re lative tu exlericil thellt service) & 14.9 & 19.6 & 22.1 & 21.6 & 12.6 & 14.7 & 12.9 & 16.0 & 11.9 & 12.9 & 12.1 & 9.5 \\
\hline (in percent of broad riveney) & 22.6 & 224 & 26.7 & 26.2 & 27.7 & 27.6 & 25.7 & 26.3 & 24.6 & 25.5 & 23.4 & 21.1 \\
\hline L'tange in real effertive exthalige rate & 18.3 & 12.5 & 7.0 & 43 & $\cdots$ & -0.4 & $\ldots$ & $\cdots$ & $\cdots$ & $\ldots$ & $\cdots$ & $\ldots$ \\
\hline 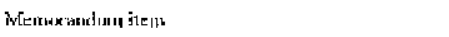 & & & & & & & & & & & & \\
\hline Numinal GDP (in millions of lek) $\mathbf{l} /$ & 425,356 & 488,511 & $5 \$ 1,282$ & 611.622 & 658.062 & 677.272 & 718,574 & 739,048 & 786,007 & 810,276 & 390,340 & 976,1002 \\
\hline
\end{tabular}

Sources: Albeniar authoritits; and Fusd staf exlinhates and projections.

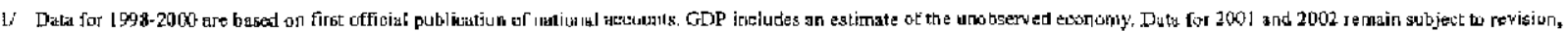
pending the chrmigilariom of official National Aceorurs.

2) Cursent account excluding official transfers.

3. Kevente minus currem expenditur.

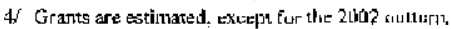

S/ [rc]ucling intorcst payments for banke restructuring.

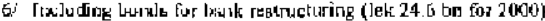

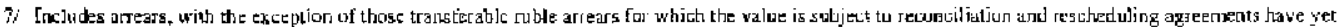
to he reached with crediturs.

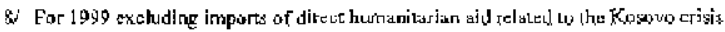


Table 3. Albania: Balance of Payments, 2000-2012

(In millions of US dollars)

\begin{tabular}{|c|c|c|c|c|c|c|c|c|c|c|c|c|c|c|c|}
\hline & $20(0)$ & 2001 & 2002 & 2002 & 20003 & qL & 42 & 93 & $q 4$ & 2003 & 2004 & 2005 & 2006 & 2010 & 2012 \\
\hline & \multicolumn{4}{|c|}{ Sk $01-03$} & \multicolumn{4}{|c|}{ R $01-09$} & \multicolumn{7}{|c|}{ Projections } \\
\hline Curтert accoun? & -274 & -263 & +10 & -440 & .381 & -80 &. $\mid 42$ & -128 & -121 & -471 & -482 & -487 & -498 & .525 & -527 \\
\hline Trade balones & -821 & $-1,027$ & $-1,096$ & $-1,155$ & $-1,129$ & -299 & -324 & -932 & -337 & $-1,292$ & $-1,375$ & $-1,452$ & $-1,541$ & $-1,894$ & $-2,064$ \\
\hline Exports & 235 & 305 & $\$ 09$ & $3: 50$ & 341 & Ins & 94 & 94 & 94 & 391 & 430 & 473 & 521 & 763 & 923 \\
\hline Insports & 1,076 & $1,3,32$ & 1,405 & 1.485 & 1,470 & $4 \mathrm{mg}$ & 419 & 426 & 431 & 1.683 & 1.806 & 1,926 & 2,062 & 2.657 & 2.987 \\
\hline Services and ineome & I11 & 224 & 138 & 120 & 160 & 42 & 35 & 42 & 41 & 139 & 184 & 214 & 232 & 390 & 4 (1) \\
\hline of which: Interest due & 12 & 14 & 26 & 21 & 24 & 4 & 7 & 4 & 7 & 22 & 27 & 31 & 37 & 88 & 100 \\
\hline Private transfors & 439 & $54]$ & 549 & 597 & 582 & 173 & 148 & 162 & 175 & $6 \sqrt{3}$ & 709 & $7 \$ 2$ & 812 & 1,099 & 1.127 \\
\hline Capital account & 319 & 282 & 135 & 362 & 371 & 98 & 97 & 103 & 117 & 416 & 420 & 452 & 443 & $47 !$ & 531 \\
\hline Offieial transfers & $11 \mathrm{~L}$ & 126 & 115 & 126 & 119 & 35 & 38 & 27 & 34 & 134 & 138 & 135 & 132 & 116 & 115 \\
\hline Dircet inveatment & 143 & 204 & 156 & 135 & 156 & 40 & 31 & 47 & 3 & 150 & 180 & 192 & 191 & 258 & 320 \\
\hline Other eapital & -6 & .131 & -5 & 8 & .7 & g & $\exists$ & 8 & -1 & 19 & .17 & -2 & .2 & .2 & -2 \\
\hline Short-tern capiral (jincl trade credits, nat) & 21 & -3 & .1 & $\$$ & 8 & 7 & 3 & 3 & 2 & 19 & 10 & -2 & 2 & .2 & .2 \\
\hline Other finaneial flows & -30 & $-12 s$ & -4 & 2 & -15 & 2 & 0 & 5 & -3 & 4 & -27 & 0 & 0 & 0 & 0 \\
\hline of which: Change in NFA of comnuereial banks (iner-) & -30 & .123 & 45 & 42 & -57 & -1 & -5 & 0 & -3 & -9 & .27 & 0 & 0 & 0 & 0 \\
\hline Ofticjai nkediume snd long-term loans (net) & 71 & 85 & 69 & 97 & 105 & L4 & 26 & 20 & 53 & 1,13 & 119 & 127 & 112 & 99 & 98 \\
\hline New borrowing & 79 & 90 & 94 & 121 & 134 & 18 & 34 & 25 & 61 & 138 & iss & 166 & 1.75 & 184 & 194 \\
\hline Multilateral Icana & 60 & 56 & 72 & 84 & 92 & 12 & 28 & 17 & 29 & 86 & Ial & 306 & 105 & $\log$ & 112 \\
\hline World Bank & 40 & 34 & 38 & 30 & 42 & 8 & 14 & 8 & 10 & 40 & 30 & 58 & 38 & 50 & 50 \\
\hline EBRD & 2 & 4 & 10 & 11 & 14 & 2 & 4 & 3 & 6 & 14 & 14 & 14 & 14 & 18 & 20 \\
\hline Other & 18 & 18 & 24 & 22 & 36 & 3 & 9 & 6 & 13 & 32 & 37 & 34 & 34 & 40 & 42 \\
\hline Bilaterl loans & 10 & 94 & 23 & 37 & 42 & 5 & 6 & $B$ & 33 & 52 & 54 & 59 & 68 & 36 & 82 \\
\hline Amorticarion & -9 & .7 & .25 & -24 & | & -3 & -5 & -4 & -9 & -25 & .36 & .39 & .53 & .85 & .96 \\
\hline Errors and omissions $/ /$ & 44 & 109 & 94 & 159 & 0 & 25 & 32 & 2 & 11 & 70 & 40 & 40 & 40 & 40 & 40 \\
\hline Net balance & 90 & 127 & 19 & $\$ 1$ & -10 & 43 & -12 & $-2 \hat{3}$ & 7 & 15 & -22 & $s$ & .15 & .13 & 43 \\
\hline Financing requirenent & -90 & -1.27 & -19 & -81 & 10 & -43 & 11 & 23 & -7 & -15 & 22 & -5 & 15 & 13 & -43 \\
\hline Avajable financing & -90 & -127 & -49 & -81 & -19 & -43 & 12 & -1 & $\cdot 10$ & -42 & -42 & -19 & -19 & -61 & -57 \\
\hline Change in net reseryes (in crease $=-$ ) & -123 & -135 & -80 & -115 & .19 & -44 & 12 & -1 & .10 & -42 & -42 & -19 & -19 & -61 & -57 \\
\hline Change in gross reserves, (inerease $=-$ ) & -135 & -134 & -72 & -1.07 & -10 & -48 & 16 & 0 & -6 & -38 & -34 & -11 & -9 & -30 & -30 \\
\hline Use of Fund Kesources (net) & 12 & -6 & -9 & -9 & -9 & 4 & -4 & -1 & -3 & -4 & -9 & -9 & -10 & .11 & -7 \\
\hline BOP support & 29 & 4 & अ1 & 31 & 0 & 0 & 0 & 0 & 0 & 0 & 0 & 0 & 0 & 0 & 0 \\
\hline Changes in arrears (iacrease $=+$ ) $2 J$ & 4 & -29 & -283 & -279 & 0 & 1 & a & 0 & -32 & -31 & -21 & -34 & -40 & 0 & 0 \\
\hline Overdue debt forgiveness & 0 & 10 & 207 & 207 & 0 & 0 & 0 & 0 & 0 & 0 & 0 & 0 & 0 & 0 & 0 \\
\hline Rescheduling $U$ & 0 & 23 & 16 & 76 & 0 & 0 & 0 & 0 & 32 & 32 & $2 !$ & 34 & 40 & 0 & 0 \\
\hline Finanking gap & 0 & 0 & 31 & b & 29 & 0 & 0 & 23 & 3 & 26 & 63 & 14 & 34 & 74 & 14 \\
\hline IUentifted Financing & 0 & 0 & $3 !$ & 0 & 28 & 0 & 0 & 23 & 3 & 26 & 19 & 11 & v & 0 & 0 \\
\hline IMF PRGF & 0 & 0 & 0 & 0 & II & 0 & 0 & 6 & 0 & 6 & II & 1] & 0 & 0 & 0 \\
\hline EU Budgetary Sumpor & 0 & 0 & 2 & 0 & 0 & 0 & 0 & a & 3 & 3 & 0 & a & o & 0 & 0 \\
\hline WB ESACIPRSC & 0 & 0 & 0 & D & 18 & 0 & 0 & 18 & 0 & 18 & 8 & 0 & 0 & 0 & 0 \\
\hline Italy & 0 & 0 & 28 & 0 & 0 & 0 & 0 & 0 & 0 & 0 & 0 & 0 & 0 & 0 & 0) \\
\hline Remaining gap & 0 & 0 & 0 & 0 & 0 & 0 & 0 & 0 & 0 & 0 & 46 & $\exists$ & 34 & 74 & 14 \\
\hline \multicolumn{16}{|l|}{ Mlemonandum iterns. } \\
\hline Gross usabje reserves & 620 & 754 & 809 & 850 & 819 & 908 & 893 & 892 & 898 & 898 & 932 & 943 & 952 & 1,119 & 1,219 \\
\hline tmonthe of imports of goods and services) & 4.2 & 4.3 & 4.9 & 4.5 & 4.5 & $\ldots$ & $\ldots$ & $\ldots$ & $\ldots$ & 4.4 & 4.3 & 4.1 & 3.8 & 3.5 & $\ldots$ \\
\hline Trade balance (percent of GDP) & .21 .4 & .24 .1 & .23 .7 & .23 .9 & -22.8 & $\ldots$ & $\ldots$ & $\ldots$ & $\ldots$ & -13.3 & -22.9 & -22.0 & -21.3 & -18.5 & .17 .2 \\
\hline Current acoosort (percent of GDP) & -7.1 & -6.2 & -8.9 & -9.1 & -7.7 & $\ldots$ & $\ldots$ & ... & $\ldots$ & .8 .5 & $.8,0$ & -7.4 & -6.9 & $-\$ 11$ & .4 .4 \\
\hline Currest acpouktt (Derocnt of GDP' inct, offic jal tranfers) & -4.2 & -3.2 & -6.4 & -6.6 & -5.3 & $\ldots$ & $\cdots$ & $\ldots$ & $\ldots$ & 6.1 & -5.7 & -5.3 & -5.1 & -40 & -3.4 \\
\hline Debt service (percent of exports of goods and services) & 4.0 & 4.2 & 7.8 & 6.4 & 7.1 & $\ldots$ & $\ldots$ & $\ldots$ & $\ldots$ & 5.5 & 6.5 & 6.5 & 7.6 & 9.8 & 9.3 \\
\hline External debt stock (persentt of GDP] ]i & 30.6 & $2 B .2$ & 24.7 & 24.5 & 25.5 & $\cdots$ & $\ldots$ & $\cdots$ & $\ldots$ & 33.9 & 24.7 & 24.1 & 23.9 & 24.2 & 22.6 \\
\hline Debt servise (petcent of eentral government teverues) & 3.4 & 3.7 & 5.9 & 5.5 & 5.3 & $\ldots$ & $\ldots$ & $\ldots$ & $\ldots$ & 4.3 & 5.0 & 4.8 & 5.6 & 7.3 & 7.0 \\
\hline Merchandise exports (procent gowth) & .7 .1 & 19.3 & 1.5 & 8.4 & 10.4 & $\cdots$ & $\ldots$ & $\cdots$ & $\cdots$ & 18.5 & 100 & 10.0 & L0. 0 & 10.0 & 10.0 \\
\hline Merclandise imports (percent growth) & 14.8 & 23.7 & 5.6 & 11.5 & 4.6 & $\ldots$ & $\ldots$ & $\ldots$ & $\ldots$ & 13.4 & 7.3 & 6.6 & 7.1 & 6.0 & 6.0 \\
\hline Exparts (GNFS) (percent growth) & 18.8 & 19.0 & -6.7 & 0.0 & 8.1 & $\ldots$ & $\ldots$ & $\ldots$ & $\ldots$ & 126 & 8.5 & 8.2 & 84 & 9.5 & 9.3 \\
\hline Inports (GNFS) (percent growth) & 21.3 & 17.7 & 6.3 & 16.2 & 4.1 & $\ldots$ & $\ldots$ & $\ldots$ & $\ldots$ & 11.1 & 6.5 & 6.3 & 6.6 & 6.2 & 6.2 \\
\hline
\end{tabular}

Sourfes; Ministry of Finatice; Banly of Albania; donols; and Fund staff estimates and projections.

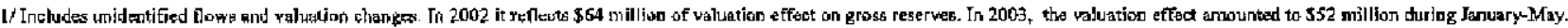

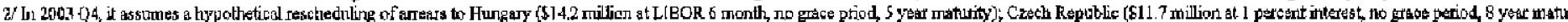

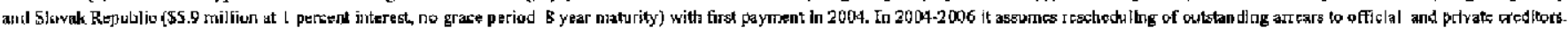
3/ Includes artears, with the cxecption of thase transfrtrable ruble arrears for which the value is subject to reconciliation. 
Table 4. Albania: Monetary Aggregales, 1999-2004 1/

(In billions of leks unless otherwise indicated; and-pcriod)

\begin{tabular}{|c|c|c|c|c|c|c|c|c|c|c|c|c|c|c|}
\hline & \multirow{2}{*}{$\begin{array}{l}2000 \\
\text { Dec. }\end{array}$} & \multirow{2}{*}{$\begin{array}{l}7001 \\
D e c\end{array}$} & \multicolumn{4}{|c|}{2002} & \multicolumn{6}{|c|}{20003} & \multicolumn{2}{|c|}{20004} \\
\hline & & & March & June & Sep. & Dec. & $\begin{array}{c}\text { March } \\
\text { SRo!fu3 } \\
\end{array}$ & March & $\begin{array}{l}\text { Junc } \\
\text { Proj, }\end{array}$ & $\begin{array}{l}\text { Scp. } \\
\text { Proj }\end{array}$ & $\begin{array}{c}\text { Dec. } \\
\text { SRol/Os }\end{array}$ & $\begin{array}{l}\text { Dec. } \\
\text { Proj. }\end{array}$ & $\begin{array}{l}\text { Mirel } 1 \\
\text { Proj. }\end{array}$ & $\begin{array}{l}\text { Tiec. } \\
\text { Proj. }\end{array}$ \\
\hline \multicolumn{15}{|l|}{ Monetary suney } \\
\hline Broad money & $32 \mathbb{E}, 1$ & $\$ 94.3$ & 993.1 & 395.3 & 406.4 & 416.7 & 432.7 & 420.6 & 429.6 & 438.3 & 4693 & 451.9 & 402.8 & 493.3 \\
\hline Currency outside banks & 99.7 & 199.1 & 124.7 & 132.7 & 129.8 & 130.8 & 136.2 & 121.3 & 124.5 & 127.9 & 1385 & 133.3 & 195.6 & 145.5 \\
\hline Deposits & 228.9 & 275.2 & 268.4 & 262.6 & 276.6 & 285.5 & 296.5 & 299.3 & 305.0 & 310.3 & 32.8 & 318.7 & 327.7 & 347.8 \\
\hline Doruestic vurreflcy tepposits & 165.3 & 186.2 & 18LG & i $7 \mathrm{ks} 9$ & 185.2 & 194,0 & 195.7 & 206.8 & 207.9 & 211.2 & 208.1 & 215.3 & 220.5 & 230.9 \\
\hline Foreign eurrency depusits & 63.6 & 89.0 & $B G . R$ & 83.7 & 91.4 & no & 100.8 & $\$ 2.6$ & 97.1 & 99.5 & $1] 4.7$ & 103.3 & 103.3 & 117.0 \\
\hline $\mathrm{M} 1 \mathrm{I}$ & 124.0 & 142.9 & 145.5 & 156.5 & {$[3,7,2$} & 152.7 & 139.9 & 144.0 & 147.9 & 151.9 & 162.5 & 158.3 & 160.5 & 172.8 \\
\hline M2 & 264.5 & 305.3 & 306.2 & 311.7 & 315.0 & 324.7 & 331.9 & 328.0 & 332.5 & 339.2 & 346.6 & 348.6 & 355.6 & 376.4 \\
\hline Net forrign assets & 130.8 & 158.6 & 159.2 & 157.8 & 165.6 & 162.1 & 177.5 & 162.9 & 167.3 & 166.3 & 1807 & 169.5 & 172.6 & 178.0 \\
\hline Bark of Albaris $2 y$ & 71.8 & 86.0 & 92.1 & 94.5 & 96,8 & 95,0 & 100.1 & $\$ 7.1$ & 99.4 & 98.9 & 101.9 & 100.2 & 101.3 & 105.1 \\
\hline Commercial Banks & 58.9 & 72.7 & 67.1 & 63.3 & 68.8 & 67.1 & 72.4 & 63.7 & 67.9 & 67.5 & 78.9 & 69.3 & 71.3 & 72.9 \\
\hline Net doriestic assets & 197.3 & 235.7 & 233.9 & 2.37 .5 & 240.8 & 254.6 & 2602 & 257.8 & 262.3 & 271.9 & 280.5 & 282.4 & 790.2 & 315.3 \\
\hline Claiss an gavernment (net of deposits) & 2133 & 231.0 & 233.5 & 225.2 & 236.7 & 245.7 & 254.0 & 246.9 & 250.5 & 256.4 & 264.8 & 263.3 & 268.0 & 280.1 \\
\hline Clains on state enterprises und farmus & 3.1 & 0.5 & 0.6 & 0.1 & 0.1 & 0.0 & 0.1 & 0.0 & 0.0 & 0.0 & 0.1 & 0.0 & 0.0 & 0.0 \\
\hline Claime on the private cector & 22.2 & 27.4 & 29.0 & 33.2 & 15.5 & 38.6 & 41.8 & 40.4 & 44.4 & 48.2 & 51.3 & $s t .8$ & $\$ 4.9$ & 67.9 \\
\hline of which: in leks & 7.5 & 4.8 & 4.4 & 3.7 & 6.9 & 8.1 & 8.1 & 8.2 & 10.0 & 11.6 & 11.3 & 13.2 & 14.8 & 20.7 \\
\hline is fotciga currency & 14.6 & 22.6 & 24.6 & 27.4 & 28.6 & 30.5 & 33.7 & 32.2 & 34.4 & 36.6 & 39.8 & 38.6 & 40.0 & 47.1 \\
\hline Other items, net & -41.3 & $-7,3.7$ & -29.2 & -309 & .31 .5 & -29.3 & -35.7 & .29 .5 & -32.7 & -32.2 & -3.9 .7 & -32.7 & -32.7 & -32.7 \\
\hline \multicolumn{15}{|l|}{ Mowetary authorities } \\
\hline Repes ve muney & 128.8 & 152.1 & 159.6 & 162.8 & 161.0 & 163.8 & 171.9 & 197.9 & 162.2 & 166.3 & 177.3 & 1726 & 175.6 & $18 B .4$ \\
\hline Program NIR (millings of U.S. d. & $\ldots$ & 342.6 & 547.4 & 545.4 & 5707 & 562.2 & 561.1 & 596.2 & 582.8 & 576.8 & $\ldots$ & 583.6 & 588.6 & 6109.1 \\
\hline Program NDA $y$ & $\ldots$ & 78.1 & 84.8 & $\$ 8.3$ & 63.0 & 87.1 & 95.3 & 76.5 & 82.6 & 87.5 & $\cdots$ & 92.9 & 99.2 & 105.2 \\
\hline \multicolumn{15}{|l|}{ Menoradidua itemst } \\
\hline Broad maney growth during the year & 12.0 & 20.2 & -0.3 & 0.3 & 3.1 & 5.7 & 2.2 & 0.9 & 3.1 & 5.2 & 8.9 & B.5 & 2.4 & 9.2 \\
\hline \multicolumn{15}{|l|}{ of which: } \\
\hline Net Eureign assets & 10.4 & 8.5 & 0.1 & -0.2 & 1.8 & 0.9 & 0.3 & 0.2 & 1.2 & 1.0 & 2.7 & 1.8 & 0.7 & 1.9 \\
\hline Net domestíc ąsets & 1.7 & 11.7 & -0.5 & 0.5 & 13 & 4.8 & 1.9 & 0.8 & 1.8 & 4.2 & 6.7 & 6.7 & 1.7 & 7.3 \\
\hline Claina on guverustielit & 4.8 & 4.8 & 0.6 & 1.6 & 1.5 & 3.6 & 1.2 & 0.4 & 1.3 & 2.7 & 3.8 & 4.4 & 1.0 & 3.7 \\
\hline Dumestically bank-financed deficit & 14.1 & 15.6 & 26 & 4.3 & 5.8 & 14.2 & 5.2 & 1.7 & 5.4 & II.3 & 16.0 & 18.1 & 4.7 & 16.8 \\
\hline in percent of CDP & 2.6 & 2.6 & 0.4 & 0.6 & 0.9 & 2.1 & 0.7 & 0.2 & 0.7 & 1.5 & 2,2 & 2.5 & 0.6 & 2.1 \\
\hline Anmial broad money growth $\left(y^{-0}-y_{1}\right.$ in petectit) & 12,0 & 20.2 & 16.3 & 13.9 & 12.1 & 5.7 & 10.3 & 7.0 & 8.7 & 7.8 & 8.9 & 8.5 & 10.0 & 9.2 \\
\hline Aluna a reserve money growth ( $y$-0-y; in percent) & 17.8 & 18.1 & 27.4 & 20.5 & 18.6 & 7.7 & 7.8 & $-4-1$ & -0.4 & 3.3 & 4.9 & 5.4 & 11.2 & 9.1 \\
\hline Annual growth in private sector eredit (y-0-y; ir perdent) & 26.9 & 43.1 & 43.5 & 48.2 & 46.3 & 32.1 & 35.1 & $\$ 1.1$ & 27.6 & 29.3 & 26.5 & 28.5 & 30.3 & 27.1 \\
\hline Aurnul $M \mid$ growth $\left\{y-\alpha-y_{\text {i }}\right.$ in percent $\}$ & 20.4 & 15.2 & 27.3 & 25.5 & 18.6 & 6.9 & 7.7 & -30 & -5.5 & -1.9 & 1.6 & 3.6 & 11.4 & 9.2 \\
\hline Aanula 247 growith $(y-0-y ;$; In percent) & 10.4 & 15.4 & 15,4 & 13.5 & 10.9 & 6.4 & 8.4 & 7.1 & 6.7 & 8.3 & 5.9 & 7.4 & 8.4 & 3.0 \\
\hline Quarterly velkcity (Quarterly GDP/BM) & 0.4 & 0.4 & 0.4 & 0.4 & 0.4 & 0.4 & 0.4 & 0.4 & 0.4 & 0.4 & 0.4 & 0.4 & 0.4 & 0,4 \\
\hline Money multiplier (absolute values) & 2.5 & 2.6 & 2.5 & 2.4 & 2.5 & 2.5 & 2,5 & 2.7 & 2.6 & 2.6 & 2.5 & 2.6 & 2.6 & 2.6 \\
\hline Currency/Broad Money natio & 30.2 & 30.2 & 31.7 & 39.6 & $3 ! .9$ & $3 \mathrm{~L} .4$ & 31.5 & 28.8 & 29.0 & 29.2 & 30.0 & 29.5 & 29.2 & 29.5 \\
\hline Currency/deposit ratio & 43.4 & 43.3 & 46.4 & 50.5 & 46.8 & 43.7 & 45.8 & 40.5 & 40.8 & 41.2 & 42.9 & 41.8 & 41.2 & 41.8 \\
\hline Currency/lek depesit ratio & nis. 0 & 64.0 & 68.7 & 74.2 & 70.1 & 67.4 & 69.6 & 58,7 & 59.9 & 60.6 & & 61.9 & $6 ! 3$ & 63.0 \\
\hline Depasits'broad money & $69.8:$ & 69.8 & 68.3 & 66.4 & 68.1 & 68.6 & 68.5 & 31.2 & 75.0 & 70.8 & 70.0 & 70.5 & 70.8 & 70.5 \\
\hline Foreign currency depositsitotel deposits & 27.8 & 32.3 & 32.4 & 31.9 & $33: 0$ & 32.2 & 34.0 & 30.9 & 36.8 & $\$ 1.9$ & 15.5 & 32.4 & 32.7 & 136 \\
\hline Lek depositstotal deposits (percent] & 72.2 & 67.7 & 67.6 & 68.1 & 67.0 & 67.8 & b6.0 & 59.1 & 68.2 & 68.1 & 64.5 & 67.6 & 67,3 & 66.4 \\
\hline FX deposits'broad moncy (pereent) & 19.4 & 22.6 & 22.1 & 21.2 & 22.5 & 22.1 & 23.3 & 22.0 & 22.6 & 22.6 & 24.9 & 22.9 & 23.2 & 23.7 \\
\hline Grins raserves (millions of U.S. dollars) 5 , & 607.8 & 754.2 & 757.9 & 807.2 & 832.9 & 860.1 & 808.8 & $90 \mathrm{SS}$ & 892.5 & 892.0 & 818.8 & 898.5 & 908.5 & 9325 \\
\hline In percent of troad noney & 264 & 26.1 & 27.7 & 28.5 & 28.5 & 27.6 & 27.1 & 27.9 & 280 & 27.5 & 25.7 & 26.8 & 26.5 & 25.5 \\
\hline US Dollar Exchange Rate (end of period) & 142.6 & 136.6 & 143.8 & 139.8 & 138.9 & 233.7 & $\ldots$ & 129.2 & $\ldots$ & $\ldots$ & $\ldots$ & $\ldots$ & $\cdots$ & $\cdots$ \\
\hline Euro Exchange Rate (end of poriud) & 130.9 & 120.3 & 125.5 & 138.1 & 136.4 & 40.8 & $\cdots$ & 140.6 & $\cdots$ & $\ldots$ & $\cdots$ & $\cdots$ & $\cdots$ & $\cdots$ \\
\hline 3-month T-bill rate (in parcest) & 7.8 & 80 & 8.7 & 9.8 & 10.3 & 11.2 & $\cdots$ & 10.8 & $\cdots$ & $\cdots$ & $\cdots$ & $\cdots$ & $\therefore$ & .. \\
\hline BoA. repo rate \{in pencent\} & $\cdots$ & 70 & 7.5 & 8.5 & 8.5 & 8.5 & $\cdots$ & 8.5 & $\cdots$ & $\cdots$ & $\cdots$ & $\ldots$ & $\ldots$ & $\cdots$ \\
\hline
\end{tabular}

Sources: Bank of Al hamiz; and Fund staft extimares.

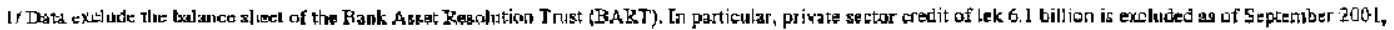
when it was transterred to the DART fronl the Savings Bank.

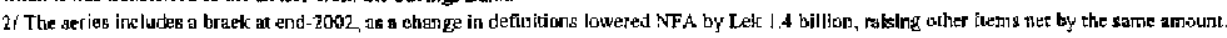

$3 /$ Derived using end-December $200 \mathrm{~L}$ exchange cates.

4 Theluding aredit trafseferred to the BART.

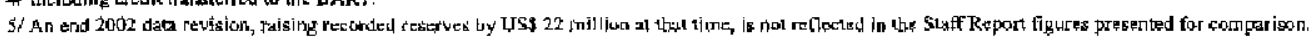


Table 5. Albania: Government Revenues and Expenditures, 1999-2003 1/

(In percent of GDP)

\begin{tabular}{|c|c|c|c|c|c|c|c|c|}
\hline & & $\begin{array}{c}1999 \\
\text { Outturn }\end{array}$ & $\begin{array}{c}2000 \\
\text { Outturn }\end{array}$ & $\begin{array}{c}2001 \\
\text { Outlurt }\end{array}$ & $\begin{array}{c}2002 \\
\text { Revised } \\
\text { Budget }\end{array}$ & $\begin{array}{c}2002 \\
\text { Revisged } \\
\text { Dullumin }\end{array}$ & $\begin{array}{l}2003 \\
\text { Original } \\
\text { budget }\end{array}$ & $\begin{array}{c}2003 \\
\text { Projections }\end{array}$ \\
\hline \multicolumn{2}{|c|}{ Total Revenue and Crants } & 24.8 & 22.9 & 22.8 & 24.0 & 22.7 & 24.5 & 23.9 \\
\hline I. & Tax Reyenue: & 17.1 & 18.9 & 19.0 & 20.4 & 19.0 & 20.8 & 20.2 \\
\hline \multirow[t]{9}{*}{$\mathbf{l . 1}$} & Tax revenues from T'ax directarate and Customs & 13.2 & 15.0 & 15.0 & 16. $\mathrm{L}$ & 14.9 & 16.0 & 15.4 \\
\hline & 1. Turnover tax/VAT & 6.1 & 6.9 & 6.7 & 7.5 & 6.8 & 7.4 & 6.9 \\
\hline & 2. Profit tax & 1.2 & 1.5 & 1.7 & 1.6 & 1.8 & 1.8 & 1.7 \\
\hline & 3. Excise tax & 1.4 & 1.7 & 1.6 & 2.0 & L.4 & 1.6 & 1.4 \\
\hline & 4. Small business tax & 0.2 & 0.3 & 0.3 & 0.4 & 0.4 & 0.4 & 0.6 \\
\hline & 5. Personal income tax & 0.6 & 0.8 & 1.0 & 1,0 & 0.9 & 1.0 & 0.9 \\
\hline & 6. National taxes $2 /$ & 1.2 & 1.4 & 1.6 & 1.4 & 1.4 & 1.5 & 1,4 \\
\hline & 7. Customs duties & 2.3 & 2.5 & 2.1 & 2.2 & 2.0 & 2.0 & 2.1 \\
\hline & 8. Road tax & 0.0 & 0.0 & 0.0 & 0.0 & 0.2 & 0.4 & 0.4 \\
\hline 1.2 & Property and local tares & 0.2 & 0.2 & 0.3 & 0.4 & 0.4 & 0.7 & 0.7 \\
\hline \multirow[t]{3}{*}{$\mathbf{I - 3}$} & Social insurance contributions & 3.7 & 3.6 & 3.7 & 3.8 & 3.8 & 4.1 & 4.1 \\
\hline & 1. Social insurance & 3.4 & 3.4 & 3.4 & 3.5 & 3.5 & 3.8 & 3.9 \\
\hline & 2. Health insurance & 0.3 & 0.3 & 0.3 & 0.3 & 0.3 & 0.3 & 0.3 \\
\hline \multirow[t]{4}{*}{ II. } & Non-tax revenue: & 4.9 & 3.0 & 3.2 & 3.0 & 3.0 & 3.0 & 3.0 \\
\hline & 1. Profil transfer from $\mathrm{BOA}$ & 3.6 & 1.9 & 1.8 & 1.4 & 1.5 & 1.4 & 1,4 \\
\hline & 2. Ineome of budgetary institutions & 1.1 & 0.9 & 0.7 & 1.0 & 0.9 & 1.1 & 1.1 \\
\hline & 3. Other & 0.2 & 0.3 & 0.7 & 0.7 & 0.6 & 0.6 & 0.6 \\
\hline \multicolumn{2}{|c|}{ 1П. Grants $3 /$} & 2.7 & 1.0 & 0.6 & 0.6 & 0.6 & 0.7 & 0.7 \\
\hline \multicolumn{2}{|c|}{ Total Expenditure } & 33.9 & 30.7 & 30.4 & 31.1 & 29,0 & 30.2 & 25.6 \\
\hline \multirow[t]{14}{*}{ J. } & Current Expenditure & 26.3 & 24.4 & 23.3 & 23.8 & 22.8 & 23.6 & \\
\hline & 1. Personnel cost & 6.3 & 6.4 & 6.7 & 6.2 & 6.2 & 6.3 & \\
\hline & a. Wages and other personnel expenditures & 4.9 & 5,0 & 5.4 & 5.0 & 4.9 & 4.8 & \\
\hline & b. Social insurance contributions & 1.4 & 1.3 & 1.4 & 1.2 & 1.3 & 1.3 & \\
\hline & 2. Interest & 7.2 & 5.6 & 4.2 & 3.7 & 3.7 & 3.7 & \\
\hline & a. Domestic & 7.1 & 5.1 & 4,0 & 3.2 & 3.3 & 3.2 & \\
\hline & b. Foreigh & 0.1 & 0.4 & 0.2 & 0.5 & 0.3 & 0.4 & \\
\hline & 3. Operations and mainteuatace & 4.9 & 4,2 & 3.8 & 2.9 & 3.0 & 2.7 & \\
\hline & 4. Subsidies 4 & 0.5 & 1.0 & 1.3 & 1.5 & 0.9 & 0.8 & \\
\hline & 5. Social insurance outlays & 5.6 & 5.7 & 5.9 & 5.8 & 5.9 & 6.1 & \\
\hline & 6. Local govenment expenditure & 1.6 & 1.4 & 1,5 & 1.8 & 1.7 & 2.0 & \\
\hline & 7. Social pratextion transfers & 1.7 & 1.6 & 1.4 & 1.4 & 1.3 & 1.3 & \\
\hline & a. Unemploymeut insurance lenefits & 0.3 & 0,3 & 0.3 & 0.2 & 0.2 & 1.1 & \\
\hline & b. Social assigtance 4/ & 1.3 & 1.2 & 1.1 & 1.1 & 1,1 & 0.2 & \\
\hline \multirow[t]{3}{*}{ IL } & Capital Expenditure & 7.6 & 6.4 & 7.0 & 7.4 & 6.2 & 6.7 & 6.7 \\
\hline & 1. Domestically finaneed & 24 & 2.8 & 3.9 & 3.5 & 2.8 & 2.9 & 2.9 \\
\hline & 2. Forcign financed projects & 3.5 & 3.0 & 3.1 & 3.9 & 3.4 & 3.8 & 3.8 \\
\hline III. & Reserve and contingency funds & $w$ & $\cdots$ & $\cdots$ & 0.5 & $\cdots$ & 0.8 & 0.8 \\
\hline \multicolumn{2}{|c|}{ Cash Balance } & -9.1 & $-7,9$ & -7.6 & -7.1 & -6.3 & -5.7 & -5.8 \\
\hline \multicolumn{2}{|c|}{ Cash Balance (exciuding grants) } & -11.8 & -8.9 & -8.2 & -7.7 & -6.5 & -6.4 & -6.4 \\
\hline \multicolumn{9}{|c|}{ Sourees of financing } \\
\hline & Domestic & 5.6 & 4.7 & 5.1 & 3.0 & 3.3 & 2.9 & 2.9 \\
\hline & Privatization receipts & 0.2 & 1,6 & 2.1 & 0.2 & 0.1 & 0.1 & 0.1 \\
\hline & Bank and nobn-banks & 5.4 & 3.1 & 3.0 & 2.9 & 3.2 & 2.7 & 2.7 \\
\hline & Fareign & 3.6 & 3.2 & 2.6 & 4.1 & 3.0 & 2.9 & 3.0 \\
\hline & Budget support & 0.9 & 1.0 & 0.2 & 1.3 & $0 . \mathrm{E}$ & 0.3 & 0.3 \\
\hline & Devejopment (gross) & 2.9 & 2.4 & 2.5 & 3.3 & 2.8 & 3.2 & 3.2 \\
\hline & mănus: prülcipal repayment & 0.2 & 0.2 & 0.1 & 0.5 & 0.4 & 0.5 & 0.5 \\
\hline
\end{tabular}

1/ The presentation of the fiscal data has been reyised since the previous staff report, to include grants as revemue xather than fioancing.

2/ Inchudes solidarily tax (discantinued in 2003) and non tox tevenuxe collected by Customs Department.

3. Information on grants are eslimates, except for the 2002 outurn.

4/ In 2001 expenditure on work incentive programs (Jek 400 million) has been reclassified under subsidies. 
Table 6. Albania: Government Revenues and Expenditurcs, 1994-2003 1/

(In millions of Lek)

\begin{tabular}{|c|c|c|c|c|c|c|c|c|}
\hline & & $\begin{array}{l}1999 \\
\text { Outtuth }\end{array}$ & $\begin{array}{l}2000 \\
\text { Ourtulth }\end{array}$ & $\begin{array}{c}2001 \\
\text { Ohtlum }\end{array}$ & $\begin{array}{c}2002 \\
\text { Revised } \\
\text { Budget }\end{array}$ & $\begin{array}{c}2002 \\
\text { Revised } \\
\text { Ontturt }\end{array}$ & $\begin{array}{c}2003 \\
\text { Original } \\
\text { Budget }\end{array}$ & $\begin{array}{l}2003 \\
\text { Projections }\end{array}$ \\
\hline \multicolumn{2}{|c|}{ Total Revenue and Grants } & 120,873 & $126,10]$ & 139,151 & 163,644 & 153,606 & 180,775 & 176,303 \\
\hline I. & 'Jax Revenue: & 83,530 & 104,098 & 116,171 & 138,987 & 128,948 & 153,887 & 149,415 \\
\hline \multirow[t]{9}{*}{ I.1 } & Tax revenues from Tax dlrectorate and Customs & $64,488$. & 82,730 & 91,629 & 109,900 & 100,635 & 118,228 & 113,756 \\
\hline & 1. Turnower tax / VAT & 29,794 & 38,107 & 41,148 & 51,076 & 46,113 & 54,584 & 50,995 \\
\hline & 2. Profit tax & 6,033 & 8,115 & 10,248 & 11,000 & 12,198 & 13,001 & 12,564 \\
\hline & 3. Excise tax & 6,961 & 9,153 & 9,544 & 13,600 & 5,324 & 11,599 & 10,347 \\
\hline & 4. Small business tax & ], $18 \mathrm{x}$ & $1,64]$ & 1,974 & 2,900 & 2,548 & 3,054 & 4,434 \\
\hline & 5. Petsonal itcomt tax & 3,110 & 4,590 & 6,300 & 6,600 & 6,149 & 7,435 & 6,652 \\
\hline & 6. National taxes $2 /$ & 5,952 & 7,576 & 9,621 & 9,800 & 9.637 & 10,882 & 10,347 \\
\hline & 7. Customs dutics & 11,450 & 13,548 & 12,794 & 14,924 & 13,387 & 14,673 & 15,461 \\
\hline & $8 \quad$ Road tax & 0 & 0 & 0 & 0 & 1,281 & 3,000 & 2,956 \\
\hline I.2 & Property and local taxes & 885 & 1,315 & 2,036 & 3,000 & 2,677 & 5,000 & 5,000 \\
\hline \multirow[t]{3}{*}{1.3} & Social insurance contributions & 18,157 & 20,053 & 22,506 & 26,087 & 25,637 & 30,659 & 30,659 \\
\hline & 1. Sacial itsubrance & 16,783 & 18,523 & 20,730 & 24,051 & 23,548 & 28,200 & 28,200 \\
\hline & 2. Health insurunes & 1,374 & 1,530 & 1,796 & 2,036 & 2,088 & 2,459 & 2,459 \\
\hline \multirow[t]{4}{*}{ II. } & Yon-1ax revenue: & 24,724 & 16,490 & 19,310 & 20,538 & 20,538 & 21,479 & 21,879 \\
\hline & 1. PTo Jit transfor fiom BOA & $17,59]$ & 10,225 & 10,912 & 9,300 & 10,291 & 10,000 & 30,000 \\
\hline & 2. Ineome of budgetary institutions & 5,352 & 4,841 & 4,383 & 6,738 & 6,302 & 7.800 & 7,800 \\
\hline & 3. Other & 1,181 & 1,424 & 4,015 & 4,500 & 3,945 & 4,079 & 4,079 \\
\hline \multicolumn{2}{|c|}{ III. Grants $3 /$} & 13,219 & 5,513 & 3,670 & 4,119 & 4,119 & 5,009 & 5,009 \\
\hline \multicolumn{2}{|c|}{ Total Expenditure } & 165,616 & 169,424 & 185,751 & 212,341 & 196,549 & 223,420 & 218,949 \\
\hline \multirow[t]{16}{*}{ I. } & Current Expenditure & 128,411 & 134,361 & 142,656 & 162,024 & 154,582 & 174,253 & \\
\hline & 1. Personncl cost & 30,737 & 35,140 & 41,210 & 42,275 & 41,942 & 46,340 & \\
\hline & A. Wages and other personnel expenditures & 23,800 & 27,720 & 32,942 & 34,185 & 33,438 & 35,659 & \\
\hline & $\begin{array}{l}\text { b. Social insurance contributions } \\
\text { 3. Wage increase }\end{array}$ & 6,937 & 7,420 & 8,268 & 8,090 & 8,504 & 9,966 & \\
\hline & 2. [ntcrest & 35,399 & 30,597 & 25,505 & 25,085 & 24,762 & 27,050 & \\
\hline & a. Domcstiv & $34,8.14$ & 28,272 & 24,429 & 21,985 & 22,468 & 23,947 & \\
\hline & b. Foreign & 585 & 2,325 & 1,076 & 3,100 & 2,294 & 3,053 & \\
\hline & 3. Operatiens and maintenance & 23,785 & 23,422 & 23,237 & 19,887 & 20,524 & 19,770 & \\
\hline & 4. Subsidies 4 f & 2.583 & 5,247 & 7,811 & 10,033 & 6,243 & 6,250 & \\
\hline & 5. Soxial insurance outlays & 27,587 & 31,375 & 36,073 & 39,836 & 40,168 & 45,032 & \\
\hline & a. Social insurance & 24,981 & 28,948 & 33,104 & 36,050 & 36,564 & 40,473 & \\
\hline & b. Healuh insurance & 2,606 & 2,427 & 2,969 & 3,786 & 3,604 & 4,559 & \\
\hline & 6. Local government expenditure & 8,038 & 7,669 & 9,404 & 12,418 & 12,230 & 14,354 & \\
\hline & 7. Social protcction transfers & 8,320 & 8,580 & 8,820 & 9,350 & 8,713 & 9,657 & \\
\hline & a. Enemployment insurance bencEits & 1,450 & 1,919 & 1,881 & 1,700 & 1,115 & $B, 180$ & \\
\hline & b. Soeial assistance 4 l & 6.360 & $6,66]$ & 6,939 & 7,650 & 7,598 & 1,477 & \\
\hline \multirow[t]{3}{*}{ II. } & Capilal Expenditure & 37,204 & 35,062 & 43,095 & $50,3: 7$ & 41,967 & 49,167 & 49,167 \\
\hline & 1. Domestically financed & 11,880 & 15,663 & 23,955 & 23,817 & 19,013 & $2], 206$ & 21,206 \\
\hline & 2. Foreign fillanced projects & 17,158 & 16,327 & 19,140 & 26,500 & 22,954 & 27,901 & 27,961 \\
\hline III. & Reserve and contingency funds & $\ldots$ & $\cdots$ & $\ldots$ & 3,140 & $\ldots$ & 5,800 & 5,800 \\
\hline \multicolumn{2}{|c|}{ Cash Balance } & $-44,588$ & $.43,323$ & $-46,600$ & $.48,697$ & $-42,943$ & $-42,645$ & $-42,647$ \\
\hline \multicolumn{2}{|c|}{ Cash Balance (excluding grants) } & $-57,607$ & $-48,436$ & $-50,270$ & $-52,816$ & 47,063 & $-47,654$ & $.47,655$ \\
\hline \multicolumn{9}{|c|}{ Sourtes of financing } \\
\hline & Domcstic & 27,389 & 25,986 & 31,102 & 20,569 & 22,383 & 21,143 & $2\rfloor, 143$ \\
\hline & Privalization teceipls & 906 & 8,975 & 12,683 & 1,127 & 485 & 1,077 & $\mathrm{~J}, 077$ \\
\hline & Bank and non-banks & 26,483 & 17,012 & 18,419 & 19,442 & 21,898 & 20,066 & 20,066 \\
\hline & Foreign & 17,199 & 17,336 & 15,498 & 28,128 & 20,560 & 21,502 & 21,504 \\
\hline & Budget support & 3,973 & 5,316 & 846 & 9,047 & 4,032 & 1,897 & 2,232 \\
\hline & Development (gross) & 14,226 & 13,019 & 15,470 & 22,381 & 19,160 & 23,650 & 23,317 \\
\hline & minus: principal repayment & 1,000 & 999 & 818 & 3,300 & 2,632 & 4,045 & 4,045 \\
\hline
\end{tabular}

I/ The presentation of the fiscal data has boen revised since the previous staff report, to include gramts as revenue rather than financing.

2f Includes solidarity tax (diseontinued in 2003) and non tax rewenue collecked by Customs Department.

3. Infonnation on grants are estimiates, except for the 2002 outurn.

4) Ir 2001 expenditure on work incentiwc programs (lek 400 million) has becn reclassified under subsidies. 
Table 7. Albania: External Debt Stock by Creditors, 1995-2003 1/ (In millions of U.S. dollars; end of period)

\begin{tabular}{|c|c|c|c|c|c|c|c|c|c|}
\hline & 1095 & 1996 & 1997 & 1998 & 1999 & 2000 & 2001 & 2002 & 20031 \\
\hline Total & 787 & 845 & 877 & 1007 & 1109 & 1173 & 1199 & 1183 & 1216 \\
\hline Multilateral & 182 & 208 & 221 & 304 & 419 & 492 & 523 & 659 & 679 \\
\hline EBRD & 5 & 10 & 9 & 9 & 8 & 8 & 9 & 14 & 15 \\
\hline $\mathrm{E}[\mathrm{B}$ & 0 & 0 & 0 & 1 & 15 & 27 & 35 & 50 & 51 \\
\hline IDA & 109 & 137 & 148 & 220 & 296 & 345 & 366 & 476 & 488 \\
\hline IDB & 0 & 0 & 0 & 1 & 1 & 2 & 5 & 10 & 10 \\
\hline IFAD & 2 & 5 & 5 & 8 & 12 & 13 & 15 & 21 & 22 \\
\hline IMF & 66 & 54 & 56 & 62 & 80 & 89 & 84 & 77 & 82 \\
\hline OPEC & 0 & 1 & 2 & 4 & 6 & 7 & 8 & 12 & 12 \\
\hline Eilateral & 145 & 170 & 183 & 218 & 211 & 214 & 259 & 398 & 410 \\
\hline Paris Club & 128 & 142 & 144 & 164 & 145 & 142 & 150 & 238 & 249 \\
\hline Pre cut-off & 47 & 41 & 32 & 27 & 20 & 18 & 17 & 55 & 55 \\
\hline Austria & 9 & 8 & 6 & 4 & 3 & 2 & 1 & 1 & 1 \\
\hline Britain & 0 & 0 & 0 & 0 & 0 & 0 & 0 & 0 & 0 \\
\hline France & 20 & 17 & 13 & 10 & 8 & 5 & 4 & 3 & 3 \\
\hline Germany & 15 & 13 & 11 & 10 & 8 & 5 & 7 & 6 & 6 \\
\hline Ituly & 2 & 2 & 2 & 1 & 1 & 6 & 5 & 5 & 5 \\
\hline Necherlands & 1 & 1 & 1 & I & 0 & 0 & 0 & 0 & 0 \\
\hline Russia & 0 & 0 & 0 & 0 & 0 & 0 & 0 & 39 & 39 \\
\hline Post cut-of[ & 81 & 102 & 112 & 137 & 125 & 124 & 133 & 183 & 193 \\
\hline Austria & 4 & 6 & 5 & 5 & 5 & 4 & 4 & 2 & 2 \\
\hline Germany & 31 & 40 & 46 & 58 & 50 & 50 & 53 & 72 & 77 \\
\hline Italy & 46 & 56 & 61 & 74 & 71 & 69 & 76 & 110 & 115 \\
\hline Non-Paris Club & 17 & 28 & 39 & 55 & 66 & 72 & 109 & 160 & 161 \\
\hline China & 0 & 0 & 2 & 2 & 2 & 2 & 2 & 41 & 41 \\
\hline Greece & 0 & 0 & 0 & 11 & 11 & 11 & 12 & 14 & 15 \\
\hline Japan & 11 & 9 & 17 & 21 & 31 & 29 & 26 & 28 & 29 \\
\hline Kuwait & 7 & 12 & 13 & 15 & 16 & 15 & 16 & 23 & 24 \\
\hline Norway & 0 & 3 & 3 & 3 & 3 & 11 & 26 & 26 & 25 \\
\hline Sweden & 0 & 4 & 4 & 4 & 4 & 4 & 4 & 4 & 4 \\
\hline Turkey & 0 & 0 & 0 & 0 & 0 & 0 & 23 & 23 & 23 \\
\hline Atrears & 460 & 467 & 473 & 484 & 478 & 467 & 417 & 126 & 127 \\
\hline Convertible currency & 133 & 134 & 134 & 134 & 134 & 131 & 91 & 56 & 56 \\
\hline Budgetary & 59 & 60 & 60 & 60 & 60 & 57 & 21 & 17 & 17 \\
\hline Bilateral (USD) clearing accoun & 74 & 74 & 74 & 74 & 74 & 74 & 71 & 40 & 40 \\
\hline Non-convertible currency 2 & 168 & 168 & 168 & 171 & 171 & 160 & 161 & 16 & 16 \\
\hline Complercial & 52 & 52 & 52 & 52 & 39 & 37 & 34 & 33 & 33 \\
\hline Cumulative Overdue Interest 3/ & 106 & 112 & 119 & 127 & 134 & 1399 & 130 & 21 & 21 \\
\hline \multicolumn{10}{|l|}{ Memuraadum items: } \\
\hline Total debt (percent of GDP) & 32.5 & 27.9 & 39.4 & 35.8 & 31.2 & 30.6 & 28.2 & 24.5 & 22.0 \\
\hline Total arrears (percent of ODP) & 19.0 & 15.4 & 21.3 & 17.2 & 13.5 & 12.2 & 9.8 & 2.6 & 2.3 \\
\hline
\end{tabular}

Sources: Ministy of Finance; Bank of Albania; and Fund staff estimates.

1/ As at end-May 2003.

2/ It consists of bilateral clearing accounts in rubles (excluding trasferable ruble anears the value of which is subject to reconciliation), converted using old official cross-txchange rates.

3/ Amended from proviously reported arrears to reflect overdue interests arising from outstanding arrears. 
Table 8. Albania: Exterial Financing Requirement and Sources, 2000-2006

(In millions of L'S. dollars)

\begin{tabular}{|c|c|c|c|c|c|c|c|}
\hline & 2000 & 2001 & 2002 & 2003 & 2004 & 2005 & 2006 \\
\hline & & & & \multicolumn{4}{|c|}{ Projections } \\
\hline Current account & 274 & 263 & 441 & 471 & 482 & 487 & 498 \\
\hline Amortization & 16 & 21 & 37 & 34 & 45 & 47 & 63 \\
\hline of which: IMF & 7 & 14 & 14 & 9 & 9 & 9 & 10 \\
\hline Change in reserves (increase -+ ) & 135 & 134 & 107 & 38 & 34 & 11 & 9 \\
\hline Reduction in arrears & -4 & 29 & 279 & 31 & 21 & 34 & 40 \\
\hline Total financing requirement & 420 & 446 & 864 & 574 & 582 & 579 & 610 \\
\hline Available financing & 391 & 409 & 550 & 516 & 496 & 530 & 536 \\
\hline Official grants & 111 & 126 & 121 & 134 & 138 & 135 & 132 \\
\hline Forcign direct investment & 143 & 204 & 135 & 150 & 180 & 192 & 191 \\
\hline Short-lemm private tirancing flows & -6 & -131 & 8 & 19 & -17 & -2 & -2 \\
\hline Official medium- and long-term loans & 79 & 90 & 121 & 138 & 155 & 166 & 175 \\
\hline Mullilateral & 60 & 56 & 84 & 86 & 101 & 106 & 106 \\
\hline Bilateral & 19 & 34 & 37 & $\$ 2$ & 54 & 59 & 68 \\
\hline Other loans & 0 & 0 & 0 & 0 & 0 & 0 & 0 \\
\hline IMF disbursetnents & 19 & 12 & 5 & 6 & 0 & 0 & 0 \\
\hline Other $1 /$ & 44 & 108 & 159 & 70 & 40 & 40 & 40 \\
\hline Financing gap $=$ I-II & 29 & 37 & 314 & 58 & 86 & 49 & 75 \\
\hline Debt rescheduling & 0 & 33 & 283 & 32 & 21 & 34 & 40 \\
\hline Balance of payments support & 29 & 4 & 31 & 0 & 0 & 0 & 0 \\
\hline Remaining gap & 0 & 0 & 0 & 26 & 65 & 14 & 34 \\
\hline Identified financing (provisional) & 0 & 0 & 0 & 26 & 19 & $1 \mathrm{l}$ & 0 \\
\hline of which: IMF PRGF & 0 & 0 & 0 & 6 & 11 & 11 & 0 \\
\hline of which: EU Budgetary Support & 0 & 0 & 0 & 3 & 0 & 0 & 0 \\
\hline of which: WB SAC/PRSC & 0 & 0 & 0 & 18 & 8 & 0 & 0 \\
\hline Unfinarced gap & 0 & 0 & 0 & 0 & 46 & 3 & 34 \\
\hline
\end{tabular}

Sources: Ministry of Finance, Bark of Albania, donors, and Fund staff estimales.

1/ It includes errors and omissions. 
Table 6. Albania: Indicators of External and Financial Vulnerability, 1995-2003

(It) percent of GDP, unless otherwise indicated)

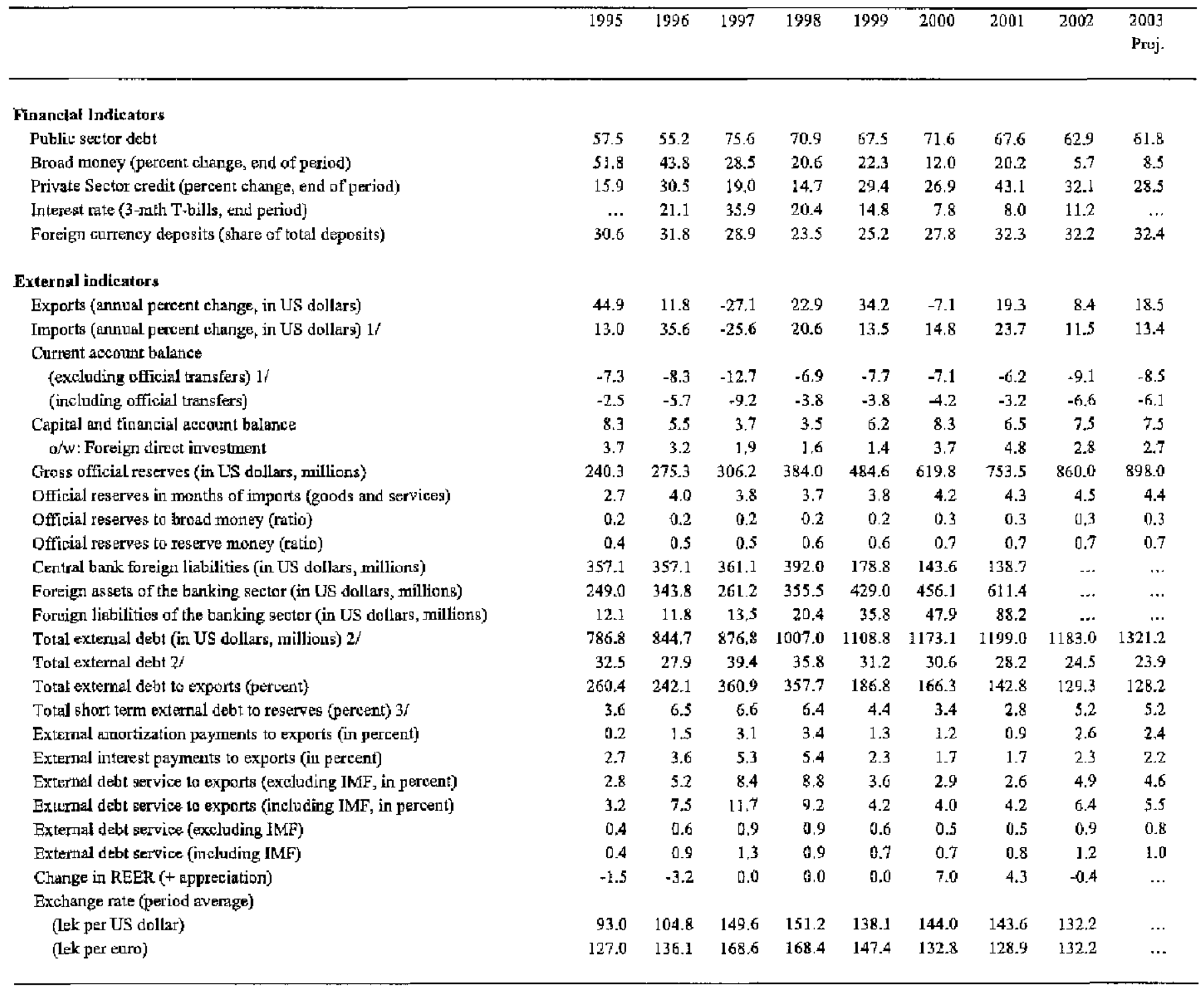

Seurces: Ministry of Einance; Bank of Albania; donors; and Fund staff estimales and projections.

1/ Exchudes imports (official transfers) related to the Kosuro crisis.

2f Includes arrears, with the exception of those transferable nuble arrears for which the valne is subject to reconciliation und rescheduling agreements have yel to be reached with creditors.

3/ Kesidual maturity basis; Albania has no short-term original maturity extemal debt. 
Table 10. Albania: Projected Payments to the Fund as of April 30, 2003

(In millions of SDRs)

\begin{tabular}{|c|c|c|c|c|c|c|c|c|c|c|c|c|}
\hline & $2003 \mathrm{l} /$ & 2004 & 2005 & 2006 & 2007 & 2008 & 2009 & 2010 & 2011 & 2012 & Beyond & Total \\
\hline \multicolumn{13}{|l|}{ Obligations from existing drawings } \\
\hline \multicolumn{13}{|l|}{ 1. Principal } \\
\hline PRGF Repsyments & 4.4 & 6.4 & 6.2 & 7.6 & 9.4 & 9.6 & 7.9 & 5.1 & 3.0 & 1.2 & 0.4 & 61.2 \\
\hline \multicolumn{13}{|l|}{ 2. Charges and Interest $2 /$} \\
\hline PRGF Itterest & 0.3 & 0.3 & 0.2 & 0.2 & 0.2 & 0.1 & 0.1 & 0.0 & 0.0 & 0.0 & 0.0 & 1.4 \\
\hline SDR Net Charges & -4.3 & 0.9 & 0.0 & 0.0 & 0.0 & 0.0 & 0.0 & 0.0 & 0.0 & 0.0 & 0.0 & -0.3 \\
\hline Total Obligations & 4.4 & 6.7 & 6.4 & 7.8 & 9,6 & 9.7 & 7.9 & 5.2 & 3.0 & 1.2 & 0.4 & 62.4 \\
\hline (percent of quota) & 9.0 & 13.8 & 13.1 & 160 & 19.7 & 19.9 & 16.2 & 10.7 & 6.2 & 2.5 & 0.8 & 128.1 \\
\hline \multicolumn{13}{|l|}{ Obligations from prospective drawings } \\
\hline \multicolumn{13}{|l|}{ 1. Prineipa! } \\
\hline PRGF Repayments & 0.0 & 0.0 & 0.0 & 0.0 & 0.0 & 0.0 & 1.2 & 2,8 & 4,0 & 4.0 & 8.0 & 20.0 \\
\hline \multicolumn{13}{|l|}{ 2. Charges and Interest $2 /$} \\
\hline PRGF Itterest & 0.0 & 0.0 & 0.1 & 0.1 & 0.1 & 3.1 & 0.1 & 0.1 & 0.1 & 0.0 & 0.0 & 0.8 \\
\hline Total Obligations & 0.0 & 0.0 & 0.1 & 0.1 & 0.1 & 0.1 & 1.3 & 2.9 & 4.1 & 4.0 & 8.0 & 20.8 \\
\hline (pereent of quota) & 0 & 0 & 0 & 0 & 0 & 0 & 3 & 6 & 8 & 8 & 16 & 43 \\
\hline \multicolumn{13}{|c|}{ Cumulative obligations (existing and prospective) } \\
\hline \multicolumn{13}{|l|}{ 1. Principal } \\
\hline PRGF Repayments & 4.4 & 6.4 & 6.2 & 7.6 & 9.4 & 9.6 & 9.1 & 7.9 & 7.0 & 5.2 & 8.4 & 84.3 \\
\hline \multicolumn{13}{|l|}{ 2. Charges and Interest $2 /$} \\
\hline PRGF Interest & 0,3 & 0.3 & 0.3 & 0.3 & 0.3 & 0.2 & 0.2 & 0.1 & 0.1 & 0.0 & 0.0 & 2.3 \\
\hline SDR Net Charges & -0.3 & 0.0 & 0.0 & 0.0 & 0.0 & 0.0 & 0.0 & 0.0 & 0.0 & 0,0 & 0.0 & -0.3 \\
\hline Tacal Obligations & 4.4 & 6.7 & 6.5 & 7.9 & 9.7 & 9.8 & 9.3 & 8.0 & 7.1 & 5.2 & 8.4 & 830 \\
\hline (percent of quota) & 9 & 14 & 13 & 16 & 20 & 20 & 19 & 16 & 15 & 11 & 17 & 1.70 \\
\hline Outstanding Fund credit & 60.8 & 62.4 & 64.3 & 56.7 & 47.3 & 37.6 & 26.54 & 20.6 & 13.6 & 8.4 & $\ldots$ & ... \\
\hline \multicolumn{13}{|l|}{ Memorandum items: } \\
\hline \multicolumn{13}{|l|}{$\begin{array}{l}\text { Outstanding Fund credit } \\
\text { It percent of: }\end{array}$} \\
\hline Exports of goods \& services & 8 & 8 & 7 & 6 & 5 & 3 & 2 & 2 & 1 & 1 & $\ldots$ & $\ldots$ \\
\hline External public debt & 6 & 6 & 5 & 4 & 3 & 2 & 2 & ] & 1 & 0 & $\ldots$ & $\ldots$ \\
\hline Gross official reserves & 9 & 9 & 9 & 8 & 7 & 5 & 4 & 3 & 2 & 1 & $\ldots$ & $\ldots$ \\
\hline GDP & 2 & 1 & ] & 1 & 1 & 1 & 0 & 0 & 0 & 0 & ... & ... \\
\hline Quota & 125 & 128 & 132 & 116 & 97 & 77 & 59 & 42 & 28 & 17 & ... & ... \\
\hline \multicolumn{13}{|l|}{ Total Obligations } \\
\hline \multicolumn{13}{|l|}{ In percent of: } \\
\hline Exports of goods \& services & 0.6 & 0.8 & 0.7 & 0.8 & 0.9 & 0.9 & 0.7 & 0.6 & 0.5 & 0.3 & $\ldots$ & $\ldots$ \\
\hline External public debt & 0.5 & 0.6 & 0.5 & 0.6 & 0.7 & 0.6 & 0.5 & 0.4 & 0.3 & 0.2 & $\ldots$ & $\ldots$ \\
\hline Gross official teserves & 0.7 & 1.0 & 1.0 & 1.1 & 1.4 & 1.3 & 1.2 & 1.0 & 0.8 & 0.6 & $\ldots$ & $\ldots$ \\
\hline GDP & 0.1 & 0.2 & 0.1 & 0.2 & 0.2 & 0.2 & 0.1 & 0.1 & 0.1 & 0.1 & $\ldots$ & $\ldots$ \\
\hline Quota & 9 & 14 & 13 & 16 & 20 & 20 & 19 & 16 & 15 & 11 & $\ldots$ & ... \\
\hline
\end{tabular}

1/May 1, 2003 to December 31, 2003.

2/ Projections are bascd on current interest tates for PRGF. The current SDR interest tate is assumed fot thet use of SDRs. 
Table 11. Albania: Schedule of Purchases Under PRGF Aтrangement

\begin{tabular}{|c|c|c|c|}
\hline Date & $\begin{array}{l}\text { Amounts in } \\
\text { millions of } \\
\text { SDRs }\end{array}$ & $\begin{array}{l}\text { In percent of } \\
\text { quota } 1 /\end{array}$ & Conditions \\
\hline June 2002 & 4.0 & 8.21 & Board approval of PRGF arrangement \\
\hline Jamuary 2003 & 4.0 & 8.21 & $\begin{array}{l}\text { Observance of end-September } 2002 \text { performance } \\
\text { criteria and completion of first review. }\end{array}$ \\
\hline July 2003 & 4.0 & 8.21 & $\begin{array}{l}\text { Observance of end-March } 2003 \text { performance criteria } \\
\text { and completion of second review. }\end{array}$ \\
\hline January 2004 & 4.0 & 8.21 & $\begin{array}{l}\text { Observance of end-September } 2003 \text { performance } \\
\text { criteria and completion of third review. }\end{array}$ \\
\hline July 2004 & 4.0 & 8.21 & $\begin{array}{l}\text { Observance of end-March } 2004 \text { performance criteria } \\
\text { and completion of fourth review. }\end{array}$ \\
\hline January 2005 & 4.0 & 8.21 & $\begin{array}{l}\text { Observance of end-September } 2004 \text { performance } \\
\text { criteria and completion of fifth review. }\end{array}$ \\
\hline July 2005 & 4.0 & 8.21 & $\begin{array}{l}\text { Observance of end-March } 2005 \text { performance criteria } \\
\text { and completion of sixth review. }\end{array}$ \\
\hline Total & 28.0 & 57.49 & \\
\hline
\end{tabular}

1/ Albania's quota is SDR 48.7 million. 
Albania: Fund Relations

As of April 30, 2003

I. Membership Status: Joined: 10/15/1991; Article XIV

II. General Resources Account:

Quota

Fund Holdings of Currency

Reserve position in Fund

III. SDR Department:

Holdings

IV. Outstanding Purchases and Loans:

ESAF/PRGF arrangements

V. Financial Arrangements:

$\begin{array}{lcccc}\text { Type } & \begin{array}{c}\text { Approval } \\ \text { Date }\end{array} & \begin{array}{c}\text { Expiration } \\ \text { Date }\end{array} & \begin{array}{c}\text { Amount Approved } \\ \text { (SDR Million) }\end{array} & \begin{array}{c}\text { Amount Drawn } \\ \text { (SDR Million) }\end{array} \\ \text { PRGF } & 06 / 21 / 2002 & 06 / 20 / 2005 & 28.00 & 8.00 \\ \text { ESAF/PRGF } & 05 / 13 / 1998 & 07 / 31 / 2001 & 45.04 & 45.04 \\ \text { ESAF } & 07 / 14 / 1993 & 07 / 13 / 1996 & 42.36 & 31.06\end{array}$

VI. Projected Obligations to Fund: (SDR Million; based on existing use of resources and present holdings of SDRs):

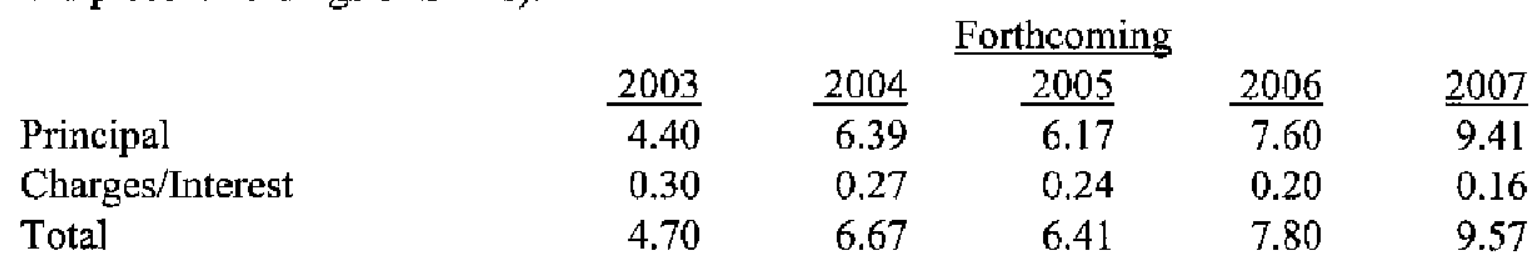

\section{Safeguards Assessments:}

Under the Fund's safeguards assessment policy, the Bank of Albania (BoA) is subject to a full safeguards assessment with respect to the PRGF arrangement, which was finalized in July 2002, and has taken into account prior safeguards work, including an assessment report issued in May 2001, and a monitoring mission conducted in March 2002. The assessment noted that the bank has made significant progress in implementing the recommendations of the first safeguards assessment, and that, in general, the bank has adecuate safeguards in place to reduce the risk of misuse and misreporting of Fund resources. Nevertheless, the assessment noted minor weaknesses in the bank's internal auditing, financial reporting, and internal control systems, all of which BoA management agreed to address. 


\section{VIII, Exchange Rate Arrangement:}

On July 1, 1992 the Albanian authorities adopted a floating exchange rate system (an independent float). Under the Fund's de facto exchange rate regime classification, Albania follows an independently floating exchange rate regime-although the monetary authorities occasionally intervene in the foreign exchange market with the objective of smoothing temporary fluctuations. Albania's exchange rate arrangement is free from exchange restrictions and multiple currency practices subject to Fund jurisdiction under Article VIII. However, the country still avails itself of the transitional arrangements under Article XIV and maintains exchange restrictions in the form of outstanding debit balances on inoperative bilateral payment agreements, which were in place before Albania became a Fund member. Albania has not imposed new restrictions under Article VIII. The unified exchange rate stood at 117 lek per U.S. dollar on May 27, 2003.

\section{Article IV Consultation:}

The conclusion of the 2002 Article IV consultation and the first review under the threeyear PRGF arrangement took place in February 2003 (Document Number EBS/03/01). Albania has moved from a 12-month cycle to a 24-month cycle, in line with the decision on consultation cycles approved on July 15, 2002.

\section{FSAP Participation and ROSCs:}

An FSAP is being planned, but no date has been set yet. A ROSC on data dissemination was published on the Fund's website in May 2000. A fiscal ROSC was completed in March 2003. Albania participates in the General Data Dissemination System (GDDS), and a complete set of GDDS metadata for the external, financial, fiscal, and real sectors, as well as for the socio-demographic indicators is posted on the Fund's Dissemination Standards Bulletin Board (http://dsbb.imf.org).

\section{Technical Assistance:}

The Fund, other multilateral organizations and donors have provided extensive assistance for institutional development in Albania. The Fund alone has sent several technical assistance missions to Albania every year since 1991. However, further institutional development is required in virtually every sector.

\section{Resident Representative:}

A Fund resident representative has been posted in Tirana since April 1993; Mr. Jan-Peter Olters has held this position since March 2002. 


\section{Albania: World Bank Relations}

\section{Parthership in Albania's Development Strategy}

The National Strategy for Socio-Economic Development (NSSED), presented to the Boards of the IMF and the Bank in June 2002 and updated in May 2003, is a comprehensive development strategy that increases the government's emphasis on poverty reduction. The NSSED has two pillars-strengthening governance and achieving high economic growthand gives priority to improving education, healthcare, and infrastructure.

The MF will continue to take the lead in assisting Albania to maintain macroeconomic stability and financial sustainability through setting quantitative targets within the framework. of the Poverty Reduction Growth Facility (PRGF) in 2002 and its subsequent review in April 2003. In addition, the IMF's structural conditionality focuses on three main categories: fiscal management and control; payments discipline; and privatization.

The Bank leads the policy dialogue in financial sector reforms, power sector reforms, social sector and pension reforms, as well as public expenditure management and public administration reform, and collaborates closely with the IMF on irnplementation.

\section{Bank Group Strategy}

The Bank's operations have been designed to support Albania's implementation of its NSSED. Therefore, in line with the objectives of the NSSED, the Bank focuses on three priorities:

- Improve governance and strengthen institutions, by strengthening transparency and accountability at all levels, increasing the contestability of the policy formulation process, and improving policy monitoring and evaluation.

- Promote sustainable private sector growth, through improving the environment for private sector development, continuing financial sector reforms, sustaining agricultural growth, improving infrastructure and promoting environmentally sustainable use of natural resources.

- Foster human development, by improving access to and quality of education and healthcare, strengthening the sustainability and equity of the social insurance system, and improving targeting and efficacy of social assistance and community social services.

Overall, the Bank's lending operations reflect a cross-sectoral emphasis on reducing poverty, strengthening institutions, and increasing transparency and accountability. To date, the Bank has approved a total of 47 operations amounting to US\$657 million, of which 23 are completed. During the next three years, the Bank will use a mix of adjustment and 
investment lending as well as country analytic work to best support the NSSED. In line with its new Country Assistance Strategy, the Bank has initiated its Poverty Reduction Support Credit (PRSC) program, and is planning 10 possible investment operations in education, health, power, transport, environment, municipal water, and community works.

The PRSC-1 was presented to the Board in June 2002 and the PRSC-2 is scheduled for Board presentation in July 2003. The PRSCs provide a strong core for the Bank's lending program and focus on multi-sectoral and thematic policy reforms that are best addressed through a single adjustment operation. The four key areas supported under the PRSCs are: promoting sustainable growth and private sector development; strengthening capacity to monitor and evaluate the policy agenda; improving service delivery and social safety net effectiveness; and improving core public functions and institutional arrangements. The PRSCs and the investment operations are complementary and mutually reinforcing: the PRSCs support reforms crucial for investment projects, while investment projects help build the capacity needed to implement reforms.

The Bank will continue work to strengthen external partnerships, particularly with the EU, which is expected to begin negotiating a Stabilization and Association Agreement with Albania in early 2003. The Bank will continue to ensure a close collaboration with the IMF in major areas of reforms.

\section{Bank-Fund Collaboration in Specific Areas}

As part of its overall assistance to Albania-through the PRSC, investment lending, and country analytic work - the Bank supports policy reforms in the following main areas, in close collaboration with the IMF:

\section{- Financial sector reforms}

Following the civil crisis in 1997, triggered by the collapse of the pyramid schemes, Albania began implementing a broad-based program of reforms in the public administration and financial sectors. The Government's reform program has been formulated as part of its NSSED and aims at divesting the State from the banking and insurance sector and at the establishment of sound governance structures in the financial institutions. Its priority has been the revival of banking sector reforms, which are now well underway. All state-owned banks, with the exception of the Savings Bank have been privatized.

The Financial Sector Adjustment Credit, approved by the Board in June 2002, will help to advance reforms in the banking sector. Measures supported by the Bank include the privatization of the Savings Bank, the development of a deposit insurance system, improvement of the bankruptcy framework and collateral enforcement, and strengthening of bank regulation and supervision, which will also assist the Government's anti-money laundering efforts. The IMF has provided technical assistance to the Bank of Albania. The IMF is also funding a resident adviser to support the implementation of the institutional development program for banking supervision developed by the Bank of Albania. 
The Government's program of reforms in the sector has been agreed in consultation with the Bank and the IMF, and the two institutions support the Grovernment's strategy. The restructuring of the financial sector is progressing well. Privatization of the Savings Bank is critical to the reform of the system. As such, it is given high priority by the Government and it is also emphasized in the PRGF program.

\section{- Power sector}

Since mid-2000, Albania has experienced a severe electricity shortage, necessitating extensive power outages despite large Government subsidies for imports of electricity. The crisis results from several factors: excessive demand caused by a chronic failure to curb illegal use and nonpayment; impact of a dry hydrological cycle on the largely hydropowerbased system; and transmission capacity constraints limiting electricity imports. Recognizing the magnitude of the crisis and its wide-ranging macroeconomic and social implications, the Government developed, in consultation with the World Bank, a two-year energy sector action plan at the end of 2000 to curtail jllegal use of electricity, improve the financial situation of KESH, reduce excessive demand and budgetary subsidies, and increase domestic generating capacity. The action plan is updated annually, and the Govemment has also adopted a Policy Statement setting out the power sector reform program.

The Bank has played the lead role in coordinating a unified stance among power sector donors on major sector issues, and has assisted the Government in shaping sector policy. Conditionality related to improving sector performance, sector restructuring, and strengthening the regulatory and legislative framework is included in the Power Sector Rehabilitation and Restructuring Project, which was approved by the Board in June 2002. The Fund-supported conditionality proposed for the three-year arrangement under the PRGF focuses on the budgetary implications and the elimination of subsidies for electricity imports by the end of the program period.

The Bank and the Fund support the Government's strategy for overcoming the crisis in the electricity sector and agree with the authorities that this will be indispensable for growth and macroeconomic stability.

\section{- Social Sectors and Pension Reform}

The Government's pension reform aims to reduce the reliance of the pension system on budgetary transfers, while addressing the overall, longer-term problems of coverage, affordability, equity, and adequacy. The pension system's deficit currently amounts to about one percent of GDP, and projections suggest that this deficit will worsen over time in the absence of reform. Compliance is low, and high social insurance contribution rates discourage willing participation in the pension scheme and in formal employment. At the same time, pensions play an important role in rural poverty alleviation and as a social safety net for the elderly. 
The Fund has stressed the importance of pension reform as a key component of medum term fiscal consolidation, and has provided policy advice to the Social Insurance Institute on the reform options available to the Government. The Bank has focused intensively on pension reform under the framework of the PRSC program, and has agreed with the Government on a comprehensive program of related parametric reforms. The first round of reforms implemented under the PRSC-1 framework included, inter alia: (i) increasing the retirement age for men and women; (ii) reducing the contribution rates for employees' pensions and other social insurance benefits; (iii) changing the base for assessing contributions designed to increase collections for employees' pensions; (iv) improving performance in collecting pension contributions; (v) narrowing the gap in pension levels for self-employed rural and urban workers, as well as increasing their levels in real terms; and (vi) raising contribution rates for self-employed rural workers, which are currently extremely low. The Bank and the Fund both fully support the approach being adopted in this sector, with the related conditionality being fully incorporated within the Bank-supported PRSC program.

Albania's ability to ensure the health and welfare of its population has been compromised by a weak physical infrastructure, major shifts in demography including significant population movements, intemal and regional instability, limited governance capacity, poor resource management and weak technical capabilities. Restructuring and reform of basic social services is therefore a priority element of poverty reduction. The Government's program aims to address under-funding in health and education by increasing budgetary allocations to these sectors under the MTEF, while also outlining reforms to improve the access and quality of education and health, as well as the targeting efficiency of social protection. The Fund has addressed a number of related measures during its regular review of the budget and the Medium-Term Expenditure Framework (MTEF). The Bank is supporting a comprehensive program of sector reforms through the PRSC, specific investment loans (for the human development sectors and public administration reform), and a program of economic and sector work. The Bank program focuses on strengthening sector management to improve the Government's ability to develop, monitor, and evaluate an effective policy agenda. The social sector conditionality is fully incorporated within the Bank supported PRSC program, with the Fund also monitoring the budgetary impacts of social sector reform as a part of its regular dialogue with the Government.

\section{- Public expenditure management}

Key challenges include: (i) ensuring that Government efforts to create a transparent budget decision making process become self-sustaining; (ii) improving the usefulness, quality, and timeliness of information upon which budget decisions are made; and (iii) enhancing budget execution transparency and accountability mechanisms, including audit mechanisms. To meet these challenges, the Government has continued to make the MTEF the centerpiece of its budget formulation process to prioritize expenditures more efficiently and to strengthen the linkages between policy objectives and budget planning. As a furst step in this process, and as part of the PRSC, the Government has closely linked the preparation of the NSSED with the preparation of the MTEF. 
To increase the effectiveness of the NSSED programs, the Government, as part of the PRSC, has committed to undertake a number of measures to further improve the public expenditure management process, including preparing an action plan for strengthening budget systems, procedures, and monitoring of budget outputs; training budget staff in line ministries; and preparing procedures for recording disbursement and expenditures on all externally-financed projects, including those financed by grants. To improve transparency and accountability the Govemment will also take steps to improve financial reporting and audit functions.

The PRGF arrangement complements the above-mentioned measures by focusing on fiscal management and control, payment discipline, and mobilizing adequate resources for expenditure priorities under the NSSED. In particular, strengthening tax and customs revenues, improving budget control of foreign-financed projects and state-owned enterprises are included in the program and deemed essential for safeguarding the programmed fiscal adjustment. Moreover, actions envisaged to regularize inter-enterprise and external arrears help address the issues of contingent budget liabilities as well as ongoing payment discipline and improved debt management. In the view of Bank staff, these measures will help maintain fiscal sustainability, and together with monetary and exchange rate policy of the PRGF program, will help ensure a sound macroeconomic environment for sustainable growth.

Prepared by World Bank staff. Questions may be addressed to Mr. Mansour Farsad at 473-2963 or Ms. Kathryn Funk at 473-5443. 


\section{Albania: Statistical Issues}

Albania has made significant progress in improving its statistical database with extensive technical assistance, including TA from the Fund. The multisector statistics mission of February 1999 secured the agreement of the authorities on an action plan for each of the major statistical areas, identifying the concrete steps and timetable for improving Albania's macroeconomic statistics. This mission was followed by several other missions in the areas identified as priority, and a resident national accounts advisor assisted the Institute of Statistics (INSTAT) in establishing a compilation system for producing GDP estimates from July to December 1999. STA fielded short-term follow-up missions in national accounts statistics during 2001 and 2002 . The authorities have thus far made progress in implementing the action plan agreed with the multisector mission as well as the recommendations of follow-up missions. The Council of Statistics has been established and its constitution and functions have been ratified. However, much still remains to be done, in particular as regards the medium-term objective of improving the compilation of the national accounts.

Albania was selected as a pilot country for the preparation of the data dissemination module of the Report on Observance of Standards and Codes (ROSC), and the ROSC was published in the Funds' website in May 2000. Albania participates in the General Data Dissemination System (GDDS), and a complete set of GDDS metadata for the external, financial, fiscal, and real sectors, as well as for the socio-demographic indicators is posted and regularly updated on the Fund's Dissemination Standards Bulletin Board (http://dsbb.imf.org). The metadata also include a comprehensive summary of plans for improving data compilation and dissemination across all statistical sectors, including socio-demographic indicators.

\section{Real Sector}

\section{A. Price Statistics}

STA has provided considerable technical assistance to Albania on price statistics. The consumer price index (CPI) constructed by INSTAT meets international standards. It is reported regularly for publication in the Albania page in International Financial Statistics (IFS). In 2001, a revision of CPI weights was initiated by INSTAT based on a new household budgetary survey. With assistance from STA a revised CPI was finalized on February 2002. As regards the producer price index (PPI), the technical assistance from STA on the PPI had been stalled during the civil disorder and resumed since early 2000. STA missions of May 2000 and January 2002 assisted INSTAT in finalizing the development of the PPI for Albania. The official monthly PPI has been published on a quarterly frequency since March 2002.

\section{B. National Accounts}

Until recently, and in the absence of official national accounts aggregates since 1990, Fund staff relied on their own estimates prepared in consultation with the authorities. These estimates were based on very partial data on (gross) agricultural output, activity in state industrial production, and extremely limited information on private sector activity. However, national accounts for 1996-2000 were released in January 2003 (see below). 
The STA resident advisor on national accounts assisted INSTAT to establish a compilation system for producing GDP estimates using existing and new data sources. As a result, preliminary annual GDP estimates for 1996-98 using both the production and expenditure approaches at current and constant prices were produced.. In addition to coverage problems, these estimates suffer from the poor quality of the source data and the lack of appropriate price indicators for deflating current price estimates. To improve basic data sources, the mission recommended changes to the design of new surveys and advised the authorities on establishing a framework within which the collaboration among government agencies involved in statistics could be institutionalized. The STA mission of January 2001 assisted the INSTAT to further improve and develop national accounts statistics in accordance with the System of National Accounts 1993 (1993 SNA). The mission worked with the authorities in improving the annual GDP estimates for 1996-99 by using the results of the recent survey and of the recently available 1999 PPI results. However, the compilation of GDP estimates continues to be hampered by weaknesses in basic data sources and incomplete coverage of the private sector. A follow-up STA mission in April 2002 recommended further enhancements and agreed on an action plan aimed at dissemination of the annual GDP data by end-December 2002. INSTAT published the first official GDP estimates covering 19962000 in January 2003.

STA has recently prepared a proposal for a technical assistance project for which the Italian government has tentatively agreed to commit $\$ 500,000$ from its subaccount with the IMF over a period of two years. The project will be put up for competitive bid from advanced national accounts agencies and represents an innovation in the way STA contracts technical assistance by promoting a fixed-price contract approach. The proposed project is aimed at further improving the national accounts estimates and basic source data. The project identifies the required technical assistance inputs (from the authorities, the technical assistance provider) and the indicators of implementation of the project.

\section{External Trade}

Data collection suffered extensively during the 1997 crisis as a number of customs posts were damaged or destroyed, and there was most likely a large temporary increase in the volume of unreported transactions. After some delays due to the Kosovo crisis, the compilation and dissemination of foreign trade indices has started again. Plans for improving the quality of trade statistics include the forthcoming introduction of the Automated System of Customs Data (ASYCUDA) with EU assistance.

\section{Government Finance}

Albania reported fiscal data (for 1995) for the first time in 1996 for publication in the 1996 Government Finance Statistics Yearbook and IFS. In March 1998, the authorities started to publish a new quarterly bulletin of government statistics. The STA multisector statistics mission in 1999 assisted in upgrading the quality of the data reported in this publication to GFSM 1986 standards and developing a system for the regular and timely reporting of data to the Fund for publication. Data for 1997 and 1998 have since been reported for inclusion in the GFS Yearboak; although data for 1999-2001 have not yet been reported. Some improvement in the collection of data on disbursement of foreign loans and grants has been 
achieved under the technical assistance provided by UNCTAD. A foreign debt database has been established and is nearly operational, requiring only auditing for final implementation. Nonetheless, further improvements are urgently required regarding the accuracy and timeliness of information on foreign financed capital expenditures.

\section{Monetary Accounts}

The existing data compilation framework conforms to the methodology recommended in the Monetary and Financial Statistics Manual, and data are compiled on a timely basis.

Following the multisector statistics mission's recommendation to expand the coverage of monetary statistics to include the accounts of the savings and credit associations (SCAs), in 2002, Parliament passed a law providing the necessary authority to the BoA to request the required data from the SCAs. There are currently 180-200 SCAs spread throughout the country and they have started reporting data to the BoA using a simplified report form.. However, data from SCAs are not yet included in the monetary survey.

The new plan of accounts for the commercial banks has been implemented. The commercial banks started reporting balance sheet data to the BoA in June 1999 on revised reporting forms prepared by the BoA. The money and banking statistics mission of November 1999 assisted the BoA in revising the reporting forms with a view to reducing the reporting burden of the commercial banks and ensuring consistency of the data reported to the various departments of the BoA. Efforts were being made to extend the time series of monetary data on a consistent basis.

The authorities have revised the monetary accounts of the BoA, the commercial banks, and the monetary survey from February 2001 onward to properly record the repurchase and reverse repurchase agreements. The revised data will appear in the October 2002 issue of $I F S$. The authorities are also making efforts to revise the historical data.

\section{Balance of Payments}

The data compiled by the BoA are methodologically sound, although some of the estimates need to be refined. The BoA has established data compilation procedures based on the classification system of the fifth edition of the Balance of Payments Manual (BOPM5). The BoA reports quarterly data to STA on a regular and timely basis. The BoA revised the methodology for the measurement of tourism services, principally through the introduction of surveys of travelers.

While this should improve the measurement of tourism services, further refinements are required. More generally, problems remain in the areas of service transactions and remittances, and in the monitoring of financial account transactions, foreign assistance and external debt. These problems could be addressed by strengthening existing data sources and improving estimation methods. The Albanian authorities have not yet initiated compiling data on foreign currency liquidity in line with the Data Template on International Reserves and Foreign Currency Liquidity. While the definition of data on official reserve assets in principle is consistent with that of the data Template, the data may not be adequate for 
monitoring the economy because other foreign currency assets and currency drains are not included.

\section{External Deht Statistics}

External debt statistics for government and government-guaranteed debt are compiled by the Ministry of Finance (MoF). These data are generally good. The MoF's external debt database, developed with technical assistance from UNCTAD, became fully operational in mid-2000. The external debt database ensures timely and accurate reporting of external government debt (including commitments of state-owned enterprises). However, the coverage of external debt data could be improved. There have been some irregularities in the presentation and recording of old external arrears. The External Debt Committee needs to work to improve coordination to ensure the timely and accurate reporting of the stock of external arrears and changes resulting from rescheduling agreements. The authorities do not collect data on private sector external debt and should work to extend coverage to include private sector liabilities. 
Albania: Core Statistical Indicalors

(as of June 3, 2003)

\begin{tabular}{|c|c|c|c|c|c|c|c|c|c|c|c|c|}
\hline & $\begin{array}{l}\text { Exchange } \\
\text { Rates }\end{array}$ & $\begin{array}{l}\text { International } \\
\text { Reserves }\end{array}$ & $\begin{array}{l}\text { Central } \\
\text { Bank } \\
\text { Balance } \\
\text { Sheet }\end{array}$ & $\begin{array}{l}\text { Reservel } \\
\text { Basc } \\
\text { Money }\end{array}$ & $\begin{array}{l}\text { Broad } \\
\text { Money }\end{array}$ & $\begin{array}{l}\text { Interest } \\
\text { Rates }\end{array}$ & $\begin{array}{l}\text { Consumer } \\
\text { Price } \\
\text { Index }\end{array}$ & $\begin{array}{l}\text { Exports' } \\
\text { Imports }\end{array}$ & $\begin{array}{l}\text { Current } \\
\text { Account } \\
\text { Balance }\end{array}$ & $\begin{array}{l}\text { Overall } \\
\text { Government } \\
\text { Balance }\end{array}$ & GDP/GNP & $\begin{array}{l}\text { External } \\
\text { Public } \\
\text { Debt/Debt } \\
\text { Service } \\
\end{array}$ \\
\hline $\begin{array}{l}\text { Date of Latest } \\
\text { Observation }\end{array}$ & June 2 & Apr 30 & Apr & Apr & March & May 15 & Apr & $\operatorname{Mar} 2003$ & $2003 \mathrm{Q} 1$ & March 2003 & 2002 & $2003 Q 1$ \\
\hline Datc Reccived & June 2 & May 29 & May & May & Apr & May 15 & May & May & May & Apr & Apr & June \\
\hline Frequency of Data & Daily & Daily & Monthly & Monthly & Monthly & Bi-weekly & Monthly & Monthly & Qzarterly & $\begin{array}{l}\text { Weeklyi } \\
\text { Monthly }\end{array}$ & Annual & Quarterly \\
\hline Frequency of Reporting & Daily & Daily & Monthly & Monthly & Monthly & Bi-weekly & Monthly & Monthly & Variable & $\begin{array}{l}\text { Weeklyf } \\
\text { Monthly }\end{array}$ & Annual & Variable \\
\hline $\begin{array}{l}\text { Frequency of } \\
\text { Publication }\end{array}$ & Daily & Monthly & Monthly & Monthly & Monthly & Bi-weekly & Monthly & Monthly & Variable & Variable & Annual & -- \\
\hline Source of Update & $\mathrm{BoA}$ & $\mathrm{B} O \mathrm{~A}$ & BoA & BoA & BoA & $\mathrm{B} \circ \mathrm{A}$ & MNSTAT & $\begin{array}{c}\text { Customs/ } \\
\text { BoAINSTAT }\end{array}$ & $\mathrm{B} \cap \mathrm{A}$ & BOA/MoF & $\begin{array}{l}\text { Estimated in } \\
\text { consultation } \\
\text { with the } \\
\text { authorities }\end{array}$ & $\begin{array}{l}\text { MoFf } \\
\text { Donors }\end{array}$ \\
\hline Mode of Reporting & Fax & Fax & E-mail & E-mail & $\begin{array}{l}\text { E-mailf } \\
\text { Pouch }\end{array}$ & $\begin{array}{l}\text { E-mai]/ } \\
\text { Pouch }\end{array}$ & Fax & $\begin{array}{l}\text { E-mail/ } \\
\text { Mission }\end{array}$ & Mission & Fax/Mission & Mission & Mission \\
\hline Confidentiality & UR & UR & UR & UR & UR & UR & UR & UR & UR & UR & UTR & UR \\
\hline
\end{tabular}

List of abbreviations:

BoA: Bank of Albania

MoF: Ministry of Finance

INST AT: Statistical Agency of the Republic of Albania

UR: Unrestricted use 
Tirana, June 17, 2003

Mr. Horst Köhler

Managing Director

International Monetary Fund

Washington, D.C. 20431

Dear Mr. Köhler,

1. The Poverty Reduction and Growth Facility (PRGF) arrangement, which was approved in June 2002, has been instrumental in promoting macroeconomic stability and economic growth. All quantitative performance criteria under the program have been observed. We regret, however, that some delays have occurred in implementing structural reforms. We have addressed coordination problems that hampered the implementation of reforms during the initial phase of the program, and are making progress in bringing the reform program back on track.

2. The attached supplementary Memorandum of Economic and Financial Policies (MEFP) and the Technical Memorandum of Understanding describe our economic program for the period ahead. We have also separately submitted to the Boards of the IMF and the World Bank our first Annual Progress Report of our National Strategy for Socio-Economic Development (NSSED).

3. In order to safeguard priority spending during the second half of 2003 in the face of possible revenue shortfalls, and as a prior action for the completion of the second review, we are developing expenditure contingencies and present these to Fund staff. Based on these actions, our policy proposals, and our commitment to strengthen performance under the arrangement further, we request completion of the second review under the PRGF arrangement as well as of the financing assurances review.

4. The Government and the Bank of Albania believe that the policies outlined in the attached MEFP provide a sound basis for achieving our program targets. During the period of this arrangement, Albania will consult with the IMF on the adoption of any further measures that may be appropriate, at the initiative of the Government or Bank of Albania, or whenever the Managing Director of the IMF requests such a consultation. In addition, the authorities will provide the IMF with such information as the IMF may request in connection with the implementation of the program. We will continue to conduct bi-annual reviews with the Fund under the PRGF arrangement, the third before end-January 2004 and the fourth before end-July 2005 .

5. Moreover, after the period of this arrangement and while Albania has outstanding financial obligations to the IMF arising from loan disbursements under this arrangement, 


\begin{abstract}
Albania will consult with the IMF from time to time on economic and financial policies, at the initiative of the Government or Bank of Albania or whenever the Managing Director of the IMF requests such consultation. These consultations may include correspondence and visits of officials of the IMF to Albania or of representatives of Albania to the IMF.
\end{abstract}

Sincerely yours,

$/ \mathrm{s}$

Fatos Nano

Prime Minister
$/ \mathrm{s} /$

Kastriot Islami

Minister of Finance
$/ \mathrm{s} /$

Shkëlqim Cani

Governor, Bank of Albania 


\section{Supplementary Memorandum on Economic and Financial Policies of the Government of the Republic of Albania for 2003-04 Under a Three-Year Poverty Reduction and Growth Facility (PRGF) Arrangement}

\section{BACKGROUND}

1. This memorandum describes our economic program for the period June 2003March 2004, consistent with our November 2001 National Strategy for Socio-Economic Development (NSSED) and the May 2003 progress report. It supplements the June 2002 and January 2003 MEFPs.

2. We acknowledge the need for further improvement in implementing reforms under the program. We have made significant efforts to reverse past slippages by improving policy coordination and accelerating reform; and strengthened communication with IMF staff to provide timely updates on implementing the agreed policy measures. We have made progress in restnucturing the Savings Bank, regularizing inter-enterprise arrears, reforming tax and customs administrations, and improving data availability. Nonetheless, progress has been weaker than expected in some areas. The end-March structural benchmark (SB) for settling Albtelekom's financial claims was only partially met. Furthermore, while many arrears within the public sector, as of end-December 2001 , have been reconciled, and many bilateral MOUs have been signed, the multilateral settlement of the remaining arrears was not completed by end-March 2003 (SB). In the electricity sector, after some monthly targets for reducing losses and increasing collection had been missed, the 2002Q4 and 2003Q1 targets were met, allowing for continued donor support.

3. Macroeconomic stability has been maintained. Since end-2002, inflation has declined to below its 4 percent targeted ceiling. The return of cash withdrawn in early 2002 to banks and continued exchange rate stability signal increased confidence in the financial system. On the other hand, growth is estimated to have moderated to $4 \frac{3}{4}$ percent in 2002 , while the trade deficit remained large, at 24 percent of GDP.

4. The end-March performance criterion (PC) on net domestic credit to government (NCG) was met, but, based on preliminary data, indicative revenue targets were missed by about 6 percent. ${ }^{1}$ At end-2002, NCG slightly exceeded the program's indicative target, but owing to low realization of foreign-financed capital expenditurewhich has continued in 2003 - and lower subsidies to the electricity company KESH, the overall deficit was well below the budget. Parliament approved the closure of the duty-free shops in January (prior action for the first review); and new environmental and agricultural taxes were introduced in the context of the 2003 budget (SB).

\footnotetext{
${ }^{1}$ However, our internal targets-the seasonal pattern of which differs from the program targets- were met.
} 


\section{OB.Jectives AND Policies}

\section{A. Overall Strategy}

5. As outlined in the NSSED, our macroeconomic and structural policies are designed to improve the overall business environment to further stimulate formal private sector activity, necessary for sustainable, high-quality growth and for reducing poverty. These policies, in particular, focus on fighting corruption, strengthening institutional integrity, and improving governance. We finalized the NSSED progress report in May 2003. Monitoring and evaluation capacity is being strengthened in line ministries to improve the availability and analysis of policy-relevant data. To improve policy design and program monitoring, we will continue to address the serious deficiencies in data collection. We will improve communication and coordination among various ministries and agencies to enhance policy implementation. We will intensify and formalize our policy dialogue with the key stakeholders, including those representing the most disadvantaged groups in society.

\section{B. Macroeconomic Framework}

6. The macroeconomic and fiscal framework for 2003-06 has been updated in the context of the new medium-term expenditure framework (MTEF), and in line with NSSED policy priorities and newly available data, including the new national accounts (Table 1). Economic growth is projected to pick up somewhat to 6 percent in 2003 -aided by the expected recovery in public and private investment-a level which we consider sustainable over the 2004-06 period, assuming further improvements in the business environment and infrastructure. Inflation is expected to stay within its 2-4 percent target range in 2003 and beyond, supported by cautious monetary policies, and reserve coverage is projected to remain around $4 \frac{1}{2}$ months of imports. The overall fiscal deficit (excluding grants) is targeted to fall further to around 5 percent of GDP by 2006; and the current account deficit to about $6 \frac{1}{2}$ percent. The recent deterioration in the current account in 2002 , however, highlights the importance of addressing weaknesses in foreign investment and the export sector.

\section{Fiscal Pollcy}

7. Fiscal policy in 2003 will be in line with the agreed budget, with an overall deficit of $6 \% / 2$ percent of GDP and domestic borrowing of $23 / 4$ percent of GDP. Tax revenues are targeted to increase by about 15 percent relative to the actual 2002 outcome, to about 21 percent of GDP, based on both policy measures and improvements in tax and customs administration. The shortfall in revenues in Q1 is expected to be at least partially reversed later in the year. Although, our current assessment is that end-year targets could still be feasible, we acknowledge the need for detailed contingency plans (see below). The budget assumes no privatization receipts from the sales of Albtelekom or the Savings Bank. Foreign financing will consist mainly of resources provided on concessional terms. We will closely monitor NCG - as defined in the Technical Memorandum of Understanding (TMU) - to avoid any slippage. We will also make further efforts to improve the management and forecasting of the liquidity needs of the government, including through a more timely 
exchange of information between the Ministry of Finance and the Bank of Albania (BoA). The 2004 budget is, preliminarily, expected to incorporate further increases in revenues, allowing a reduction in the overall deficit (excluding grants) to about 6 percent of GDP and in domestic borrowing to $2 \frac{1}{2}$ percent of GDP, assuming that GDP will grow by 6 percent.

8. We will continue to modernize our tax and customs administrations, on the basis of our medium-term revenue framework and the recommendations of the recent FAD missions. To achieve this objective, we will intensify efforts to fight fiscal evasion and smuggling. We will also enhance the transparency and relative simplicity of our tax system, and expand the provision of information to taxpayers. We will ensure that the focus on revenue targets will not compromise reform efforts or result in harassment of taxpayers. We will not introduce any fiscal scheme that would undermine tax compliance over the medium term or raise the potential for money laundering and other illegal activities.

9. Specifically, we will execute the following measures to attain the targeted revenue increase in 2003 , while ensuring a correct application of the tax Laws. Regarding tax and social insurance, we will (in line with the January 2003 MEFP):

- Gradually shift responsibilities for the collection of social insurance contributions to the General Directorate of Taxation (GDT), starting with large taxpayers (by end-September 2003, contingent on the assessment by the FAD expert in July 2003). To this end, in collaboration with the World Bank and the UK Government Department for International Development (DFID), we will strengthen cooperation between the GDT and Social Insurance Institute, including by undertaking measures to resolve administrative hurdles.

- Implement fully a new function-based organization structure in the GDT (end-July 2003). We will also establish formal tax policy and revenue forecasting units within the Ministry of Finance (MoF) (end-September 2003).

- Complete implementation of new computer administration systems for profit taxes (endSeptember 2003) and social insurance contributions (end-March 2004).

- Raise the number of registered taxpayers by over 10 percent (year-on-year) to 57,000 by end-September 2003.

In addition, we will introduce the following new measures:

- Improve procedures for VAT refund with the establishment at the Treasury of a separate account for VAT refunds, funded with around 5 percent of gross VAT receipts already collected; and require the GDT to report on VAT refunds along with the monthly publication of data on revenue collected (SB; end-June 2003).

- Examine the possible introduction of a simplified registration procedures for the small business tax as part of the 2004 fiscal package, with a once-only/permanent registration as long as the business is in operation. 
- Reduce discretionary practices by tax officials, by including instructions on tax procedures and tax laws in the official gazette and in pamphlets distributed to taxpayers (SB; end-2003).

In relation to tax policy, as planned, a new excise law will become effective by end-June, 2003, aimed at (i) bringing cigarette excise rates closer to EU standards and aligning them with the existing customs code; and (ii) enhancing administrative procedures, including for tax assessment, the appeals process, the approval process of establishing bonded warehouses for excisable goods, and search and inspection of sites suspected of evading excise taxes.

In addition we will:

- Ensure the consistency of VAT and customs duties laws to reduce discretion, in line with the recommendations of the fiscal ROSC (SB; end-2003).

- Eliminate estimated transportations costs from the base of the VAT on imports, as recommended by the fiscal ROSC.

In the area of customs administration, we will proceed with implementing the measures agreed in the January 2003 MEFP (albeit with some delays):

- Strengthen audit and control procedures in the General Directorate of Customs (GDC), by developing and implementing a strategy - in consultation with Fund staff - for combating fraud and corruption, based on effective direction to field units, an enhanced role for regional offices, improved cooperation with the GDT, and internal audits (by end-July 2003). Enhance cooperation between the GDC and GDT to fight evasion through underinvoicing.

- Adoption by the Council of Ministers of an decision to amend the GDC structure to establish a post-clearance control unit of around 10 senior inspectors, concentrating on valuation, classification, origin, exemption, and suspension claims (SB; end-June 2003).

- Complete implementation of ASYCUDA computer administration system in the Tirana custom house and Durrës port and ensure some altemative means of data transmission (end-January 2004 PC; initially an end-September SB) until both systems are brought online and the transit system with ASYCUDA is fully operational (end-March 2004).

- Maintain the quarterly reporting on corrective measures being taken to deal with problems identified by internal audit function.

\section{We will further improve relations with taxpayers and reform the appeals} procedures. As agreed before, to ensure the fair application of tax legislation, we will establish taxpayer service centers in all local branches of the GDT and GDC. As a further step, we will strengthen the tax appeals procedures, including by publishing the decisions of the appeals commission (SB; end-December 2003). To prepare and monitor this process, we 
will set up a working group, comprising representatives of the MoF, GDT, and the business community. In line with the January $2003 \mathrm{MEFP}$, we have revised the Instructions on Tax Procedures and manuals for the enforced collection of taxes (including the freezing of bank accounts) to comply with the law on Tax Procedures. Procedures for the initiation and conduct of enforced collection action will be disseminated publicly and their operation subject to oversight by the taxpayers' consultative forum will be established.

\section{Following the low realization of expenditures in 2002 , we will safeguard the} planned improvements in infrastructure and other spending priorities. In particular, we will improve the implementation of foreign financed projects; and to prepare for possible revenue shortfalls, we will develop expenditure contingencies for the second half of 2003 in close consultation with line ministries and in line with the NSSED - and submit these to IMF staff (prior action for the second review). The subsequent application of these contingencies will be determined in consultation with Fund staff, with a first assessment on the basis of the revenue data up to end-June. As specified in the January $2003 \mathrm{MEFP}$, we will continue to reorient expenditures in 2003 toward NSSED priorities:

- We intend to increase operations and maintenance expenditure for health and education by about 15 percent relative to the 2002 outturn, and, in consultation with the World Bank, introduce specific measures to improve access by the poor to health care.

- The wage bill will remain unchanged as a share of GDP, with the sectoral allocation determined in consultation with the World Bank. The quality and motivation of teachers and healthcare workers will be strengthened by salary increases of 5 percent in real terms.

- To make public administration more effective, a database on all employees is becoming fully functional.

- We will complete a World Bank-supported study on social safety nets.

- Electricity import subsidies will be limited to Lek 3.4 billion in 2003 . To increase transparency, we have included other subsidies to KESH as separate line items in the budget-specifically for the payment of arrears (Lek 1.6 billion, out of a total allocation for arrears of Lek 2 billion) and the concession agreement with DARFO (Lek 0.4 billion). In addition, to ensure the full and timely payment of current obligations by the water companies to $\mathrm{KESH}$, the budget includes an explicit subsidy to the water companies (including about Lek 0.6 billion for electricity).

- We will give local communities more decision-making authority within our program of decentralization, with further increases in grants (net of the shift of primary health care operations and maintenance to a conditional grant mechanism), but will ensure that the pace of decentralization is in line with developing administrative capacity. We will enhance transparency in communicating local government policy decisions to the public. 
- The reserve and contingency funds will be used to meet any net government obligations arising from the regularization of inter-enterprise arrears. We will otherwise use these funds sparingly and in emergencies.

12. We will improve expenditure management, based on the recommendations of the fiscal ROSC mission. To enhance the timeliness and quality of reporting foreignfinanced capital expenditures, we have issued a new instruction requiring projectimplementation units in line ministries to report on a monthly basis. The appropriation structure in the 2004 budget document will be extended to cover program classification of capital expenditure and the scope for transfers in the appropriation of one ministry to another without approval of Parliament will be significantly reduced from the current maximum of 10 percent of the total (end-December $2003 \mathrm{SB}$ ); the structure will be further expanded in the 2005 budget to include object/economic classification of recurrent expenditure. We will gradually bring forward the budget process to give Parliament more time for review. We will continue to computerize regional treasury offices to improve reporting-to be completed for the Tirana office in 2003 and for the entire project in 2004 . We will integrate all off-budget funds with the Treasury system, and end the practice of earmarking tax revenues in time for the 2004 budget.

13. We will complete the regularization of public sector inter-enterprise arrears based on a revised and detailed plan. We are progressing in recording arrears and in the reconciliation process for large enterprises, resulting in signed bilateral MOU's specifying net overdue amounts as of end-December 2001. We will present these arrears (by accounts payable and accounts receivable) in matrix form according to the format set up by the FAD technical assistance report, and before end-June 2003, will determine the arrears that can be reduced through netting or cancelled through a cash settlement using the government budget. We will establish a special unit within the Ministry of Economy in order to monitor and report on a quarterly basis to all concerned ministries the status of all old and new arrears (end-July). Before end-September we will execute the government-assisted netting and final settlement of public sector inter-enterprise arrears as of end-December 2001, and present to the Fund drafts strategies for ensuring the full and timely payment of all obligations contracted thereafter (end-September PC; initially an end-March SB).

\section{Monetary Policy}

14. A gradual easing of monetary policy is envisaged, following the tightening in 2001 and 2002 and the return of monetary stability. We reduced the repo rate by $1 / 2$ percentage point in April and will consider further gradual cuts---which appears justified by subdued inflation and lower foreign interest rates - taking into account the effects of this initial reduction, with the aim of keeping inflation within a $2-4$ percent range. The repo rate will remain our key policy instrument, and we will seek to further improve its impact on 
market rates through supportive monetary management, including open market operations. ${ }^{2}$ These operations will also be guided by our monetary program-including the targets for NDA and NLR - which aims to ensure consistency between monetary and inflation objectives and the agreed fiscal deficit target. We will proceed with plans to strengthen the effectiveness of our monetary instruments-which hinges on financial market development-and enhance their transparency, and to conduct research on the determinants of inflation, with technical assistance from the TMF's Monetary and Financial Systems Department (MFD). We will maintain a flexible exchange rate regime, with selective interventions aimed at smoothing excessive exchange rate fluctuations. We will ensure that the legal and operational independence of the BoA will not be compromised.

15. The monetary program assumes continued monetary stability, inflation in line with the target, and a reserve-to-import ratio of about $41 / 2$ months. Velocity is projected to remain fairly stable, while the rapid conversion since July 2001 of currency in circulation into domestic currency bank deposits is assumed to come to a halt now that their shares from before the bank run have been broadly restored.

16. The BoA will continue to strengthen banking supervision, in the context of the Supervisory Development Plan, developed in consultation with the World Bank. The MoF will reinforce its efforts to combat money laundering. In both areas, technical assistance will be provided by a new MFD resident advisor.

17. The BoA will strengthen its control, accounting, and auditing systems in line with the July 2002 safeguards assessment report. It will continue to cooperate with the safeguards policy and provide updated documents and data as necessary.

\section{E. Structural Policies}

18. Improving governance in general, and fighting corruption in particular, are key priorities within our broader strategy for supporting private sector activity and economic growth. In this context, policies, developed in consultations with the World Bank and the EU, include efforts to enhance the professionalism and motivation of public sector personnel; limit the scope for corruption by ensuring clear and consistent regulations and reducing discretion; and set high standarcs for public sector workers, combined with resolute action against abuse. Specific actions will include:

- Strict compliance with the Civil Service Law and related legislation and the draft code of ethics in hiring and firing policies for high level government officials; and in

${ }^{2}$ Open market operations will be conducted in line with Article 32 of the BoA Law, consistent with the objective of maintaining price stability. These operations are not subject to the 5 percent limit on the stock of T-bills under Article 30-which applies only to T-bills obtained through direct credit to the government. 
consultation with the World Bank, extending the Law to include all institutions related to government spending agencies by end-June 2004 (SB).

- Starting the enforcement of procedures to implement the law on asset declaration of public officials, developed in consultation with the World Bank, by end-September 2003 (SB). Implementation will require, inter alia, the provision of office space and adequate staffing to the new inspectorate.

- Streamlining and clarifying improperly defined provisions in the tax laws (see above).

- Submission of a more detailed budget to Parliament to enhance the transparency of expenditure management and the accountability of the executive branch (see above).

- Execution of a self-assessment of anti-money laundering legislation relative to international standards, before end-July 2003.

19. We will implement policies to improve the business environment, strengthen the export sector, and attract foreign and domestic investors, incorporating the findings of the recent study by FIAS. Our general strategy is to eliminate barriers to investment for all sectors rather than to introduce selective incentives. These barriers, as identified by FIAS, include arduous licensing and tax procedures, lack of transparency in policy implementation, abuse of power by officials, and weak law enforcement. We have established a high level task force for coordinating the associated reforms. An action plan including specific measures with clear deadlines will be finalized by end-June 2003. We have established a foreign investment promotion agency, and are currently establishing export promotion and small business development agencies, while ensuring that these initiatives do not lead to increased bureaucracy or duplication. A proposal for improving access to credit by small firms will include strong safeguards against corruption and will be presented to World Bark and IMF staff for comments before its adoption. We will continue to improve consultations with businesses, including through the Business Advisory Council. We are preparing draft legislation to avoid abuse of market power. In order to create better conditions in the agricultural sector, we will prepare a law to regulate land ownership, complete the registration of all lots by end-2004, and establish offices for land in every district.

20. In preparing for privatization, we will further restructure the Savings Bank and Albtelekom, with assistance provided by the IFC, EBRD and World Bank. In this context, we have transferred the Saving Bank's pension functions to Albapost. We will move a further part of the bank's fiscal functions to private banks, by completing the auctioning of these functions in five cities by end-2003 (SB). We have already begun a new marketing campaign. We confirm that the bank will not embark on extending credit (other than to the government) before privatization except, possibly, on a pilot basis and with the prior approval of the BoA, and World Bank and IMF staff. We will promote the continued provision of banking services throughout the country after the privatization. Regarding Albtelekom, a pre-privatization was initiated in February 2003, with IFC and EBRD participation and the dissolution of its joint venture with New World has been finalized. We will settle the company's remaining financial claims by end-September 2003 (SB, delayed 
from end-March). In the oil sector, we will bring all parts of ARMO and SERVCOM to the point of sale by end-2003.

21. We will ensure that targets under the 2003-04 electricity sector action plan are met consistently. Also, the MoF will monitor carefully the financial situation of KESH to help ensure compliance with the collection targets and to avoid unexpected financial problems-in particular, the emergence of new arrears or slippages in counterpart financing. Electricity prices were increased as of April 2003, delayed from January. The next scheduled price increase will be introduced no later than by January 1,2004. We will not introduce any additional differentiation in electricity prices for nonbudgetary and private consumers, given our objective of cost-based pricing. Further price increases for households will be accompanied by measures to alleviate the impact on vulnerable groups, to be designed in consultation with the World Bank. We remain fully committed to phasing out subsidies to KESH for electricity imports by the end of 2004 . The installation of electricity meters is key to reducing excessive electricity demand and we will complete the process by mid-2004. We will not grant any further concessions to investors that include reduced or fixed electricity tariffs. We will proceed with new electricity generation projects in consultation with the World Bank and will first focus our efforts on thermal power-in particular the construction of the planned Vlorë power plant.

22. Our financial system is being strengthened and modernized. In October 2002 , the deposit insurance scheme became operational and government notes with a two-year. maturity were introduced. To improve the functioning of financial markets, we will encourage the sale of treasury bills by commercial banks. We will also seek to develop a new marketing channel for treasury bills using Albapost. With World Bank assistance, we will strengthen the supervision of the insurance market, promote the establishment of a credit information bureau, and, by end-2003, establish an operational RTGS system. Concerning our efforts for reviving the stock market, in view of deficiencies in accounting and auditing practices, we acknowledge the need to be cautious. With World Bank assistance, we are recruiting an advisor to help prepare the strategy for trade in company shares and are working on improving auditing and accounting legislation. At this stage we are not considering a transfer of primary T-bill auctions from the BoA to the stock exchange.

\section{Bullding on the recent publication of the national accounts for 1996-2000, we} will further improve the quality and coverage of statistics, using the GDDS as a framework. We will publish full documentation of the methodology and data sources for the national accounts by end-June 2003, and will further improve their quality during 2003-04 (financed in part by a grant from the Italian government) through improving the core data sources. We are also strengthening the provision of short-term indicators of economic activity, essential for dependable policy analysis, and of our balance of payments data (with assistance provided by a May 2003 STA mission). We will continue to publish results from the Living Standard Measurement Survey 2002, which provides important survey level data (including on remittances). The new Law on Statistics, to be discussed in Parliament by endJuly 2003, will strengthen NSTAT's access to data. We will improve statistical reporting by the line ministries and other agencies to INSTAT and vice versa. We will take advantage of computerization projects in tax and customs departments to increase the quality, timeliness, 
and range of statistical data available. We will monitor the adequacy our framework for data sharing within the public sector on a regular basis. We will examine the need for a further increase in INSTAT's budget to ensure adequate staffing.

\section{F. External Policies}

24. With further efforts to encourage foreign investment and exports, we expect the balance of payments position to remain sustainable. The worsening of the current account in 2002 did not appear to reflect increased debt-creating inflows, and the outlook for FDI and the trade balance in 2003 is more favorable, with improvements on the supply side. Creating a more business-friendly environment, to attract foreign investors and strengthen exports and tourism, will be crucial to improving our external position. Although the increase in ceilings on nonconcessional borrowing in 2003 over actual in 2002 is intended to finance essential road and hospital projects, we will critically evaluate and better prioritize all planned projects to stay within reasonable limits and ensure external debt sustainability. We will strengthen evaluation and coordination of foreign-financed projects-including through quarterly reporting by line ministries on proposed foreign borrowing over a 3-year horizon.

25. We are committed to eliminating external arrears to official creditors during the PRGF-supported program. We will also enhance efforts to regularize arrears to private creditors. In 2002, we finalized the rescheduling agreements with China (post-1978) and reconciled arrears with the Czech Republic, the Slovak Republic, and Poland. Parliament approved a set of criteria for dealing with the remaining external arrears in May, and the MoF will continue quarterly reporting on these arrears (SB). To work toward accepting Article VIII obligations, we will submit to Parliament, by end-December 2003, the protocol agreements for restructuring of arrears with Hungary (new SB), as well as the Czech Republic and the Slovak Republic. We will also continue to reconcile arrears in nonconvertible currencies.

26. We will maintain a liberal trade regime and enhance regional cooperation. The free trade agreements with Macedonia and Romania have already been ratified. Those with Serbia and Montenegro, and Croatia are expected to be ratified by mid-2003. We are also discussing agreements with Bulgaria and Bosnia and Herzegovina. The negotiation on the Stabilization and Association Agreement with the EU also represents an important step toward increased outward orientation.

\section{G. Program Monitoring}

27. The third review under the PRGF-supported program will be based on end-September 2003 quantitative PC's and end-December 2003 indicative targets (Table 2 and the TMU) and the end-September 2003 and end-December 2003 structural conditionality (Table 3). PC's and indicative targets for end-March 2004 and end-June 2004 will be set at the time of the third review. During the progran period, Albania will not impose or intensify restrictions on the making of payments and transfers for current international transactions, or introduce multiple currency practices, or conclude bilateral payments agreements inconsistent with Article VIII, or impose or intensify import restrictions for balance of payments reasons. 
Table 1. Albania: Basic Indicators and Macroeconomic Framework, 1998-2006

\begin{tabular}{|c|c|c|c|c|c|c|c|c|c|c|c|c|}
\hline & \multirow[t]{2}{*}{1998} & \multirow[t]{2}{*}{1999} & \multirow[t]{2}{*}{2000} & \multirow[t]{2}{*}{$20-01$} & \multicolumn{2}{|l|}{2002} & \multicolumn{2}{|c|}{2003} & \multicolumn{2}{|c|}{2004} & 2005 & \multirow{2}{*}{$\frac{2006}{\text { Proj }}$} \\
\hline & & & & & SRO1/09 & Ess. & S.R.0 503 & Proj & SkBLOS & Eroj. & Proj. & \\
\hline & \multicolumn{12}{|c|}{ [Percent change] } \\
\hline RealGDF LI & 12.7 & 8.9 & 7.7 & 6.8 & 4.7 & 4.7 & 6.0 & c. 0 & 6.0 & 6.0 & 6.0 & 6.0 \\
\hline Retail prices (evg) & 20.9 & 0.4 & 0,0 & 3.1 & 5.6 & $\$ .4$ & 3.0 & 3.0 & 3.0 & 3.0 & 30 & 3.0 \\
\hline \multirow[t]{2}{*}{ Retail prices (end-period) } & 8.7 & -1.0 & 4.2 & 3,5 & 3.0 & 2.1 & 3.0 & 3.6 & $3 . \overline{0}$ & 30 & 3.0 & 3.0 \\
\hline & \multicolumn{12}{|c|}{ (In percent of GDP) } \\
\hline \multicolumn{13}{|l|}{ Saving-investmenı batance } \\
\hline Föręign asving $2 /$ & 6.9 & 7.7 & 7.1 & 6.2 & 8.9 & 9.1 & 7.7 & 8.5 & 7.5 & 8.0 & 7.4 & 6.9 \\
\hline Domestie saving & 6.9 & 9.1 & 13.0 & 16.4 & 10.1 & 12.7 & 11.8 & 140 & 13.9 & 13.3 & 16.0 & 16.6 \\
\hline Public 3 & -5.8 & .5 .3 & -2.5 & -1.2 & -1.0 & -0.3 & 0.1 & 0.2 & 2.3 & 1.0 & 1.2 & 1.4 \\
\hline Private & 128 & 14.9 & 15.5 & 17.6 & It.1 & 13.] & l L.7 & 13.8 & 11.6 & 14.3 & 14.8 & 15.2 \\
\hline Irveslane al & 13,6 & 16.7 & 20.0 & 22.3 & 19.0 & 21.5 & 19.5 & 22.6 & 21.5 & 23.3 & 23.4 & 23.5 \\
\hline Public & 5.6 & 5.9 & 6.4 & 2.0 & 6.5 & 6.6 & 6.7 & 5.7 & 8.3 & 7.0 & 7.0 & 7.0 \\
\hline Private & 8.0 & 10.8 & 13.7 & 15.5 & 12.5 & 15.2 & 12.8 & 15.9 & 3.2 & 16.3 & 16.4 & 16.5 \\
\hline \multicolumn{13}{|l|}{ Fiseisl secturer } \\
\hline Reverises and grants $4 /$ & 23.3 & 24.3 & 22.9 & 22.8 & 24.1 & 72.7 & 25.1 & 23.9 & 26.2 & 25.0 & 25.3 & 25.3 \\
\hline Tax revenue & 16.9 & 17.1 & 18.9 & 19.0 & 20.4 & 19.0 & $2 \mathrm{~L}-4$ & 20.2 & 22.2 & 21.2 & 21.6 & 21.6 \\
\hline Expenditures & 33.2 & 33.9 & 30.7 & 30.4 & 31.0 & 29.0 & 31.0 & 29.6 & 31.3 & 30.2 & 30.4 & 10.2 \\
\hline Prinaary & 24.8 & 26.6 & 25.2 & 26,3 & 27.2 & 25.4 & 27.2 & 26.0 & 27.3 & 26.4 & 26.7 & 26.4 \\
\hline Interest $5 /$ & 8.3 & 7.2 & 5,6 & 4.2 & 3.7 & 3.7 & 3.8 & 3.6 & 4.0 & 3.8 & 3.8 & 3.8 \\
\hline Ovetall balance (excluding grants) & -11.4 & -11.3 & -8.9 & -8.2 & -7.5 & -6.9 & 6.6 & -6.4 & -6.0 & -6.0 & -5.8 & -5.6 \\
\hline Qwerall tralance (including grants) & -9.7 & -9.1 & -7.9 & -7.6 & -6.9 & -6.3 & -6.0 & -5.8 & -5.2 & -5.2 & -5.1 & -5.0 \\
\hline Printary balasibe (including grants) & -1.2 & -1.9 & .2 .3 & $-3,4$ & $=3.2$ & -2.6 & -2.2 & -2.2 & -1.2 & -1.4 & .1 .3 & -1.7 \\
\hline Denicastie botrowing & 6.9 & 5.4 & 3.1 & 3,0 & 3.0 & 3.2 & 2.8 & 2.7 & 2.1 & 2.4 & 2.0 & 1.8 \\
\hline Privatization reneipts & 0.0 & 0.2 & 1.6 & 2.1 & 0.2 & 0.2 & 0.1 & 0.1 & 0.4 & 0.3 & 0.3 & 0.3 \\
\hline Foreign finance & 2.8 & 3.6 & 3.1 & 2.6 & 3.8 & 3.0 & 0.1 & 2.9 & 2.6 & 2.4 & 2.8 & 2.9 \\
\hline Publie Debt & 70.9 & 67.5 & 71.6 & 67.6 & 64.4 & 62.9 & 64.6 & 61.8 & 63.7 & $6 ! .7$ & 59.8 & $5 \mathrm{E} .2$ \\
\hline Tornestic 8 & 35.1 & 36.3 & 41.0 & 39,4 & 39.6 & 38.4 & 39.1 & 37.9 & 37.8 & 37.0 & 35.7 & 34.3 \\
\hline Externa! (including publialy guaranteed) ?] & 35.8 & $1 \mathrm{~L} .2$ & 30.6 & 28.2 & 24.7 & 24.5 & 25.5 & 23.9 & 25.9 & 24.7 & 24.1 & 23.9 \\
\hline \multicolumn{13}{|l|}{ Monstary indicators } \\
\hline Bruath Itasley gre with (in percant) & 20.6 & 22.3 & 12.0 & 20.2 & 7.6 & 5.7 & 8.9 & 8.5 & 8.2 & 9.2 & 9.2 & 9.2 \\
\hline Private aredit growth (in peroent) & 14.7 & 29.4 & 26,9 & 43,1 & 33.0 & 32.1 & 26.5 & 28.0 & $\ldots$ & 27.1 & $\ldots$ & $\cdots$ \\
\hline Velocity & 1.9 & 1.7 & 1.6 & 1.6 & 1.6 & 1.6 & 1.6 & 1.6 & 16 & 1.6 & 1.6 & 1.6 \\
\hline \multirow[t]{2}{*}{ Interist ate (3-mith T-bilis, end-period) } & 20.4 & 4.8 & 7.8 & 8.0 & $\cdots$ & 11.2 & $\cdots$ & $\cdots$ & $\cdots$ & $\cdots$ & $\cdots$ & $\cdots$ \\
\hline & \multicolumn{12}{|c|}{ (In millions of U.S. dulters) } \\
\hline Extemal sector & & & & & & & & & & & & \\
\hline Trade balance $\mathbf{~}$ & $-62 !$ & -663 & -821 & .1027 & -1096 & -1155 & -1129 & -1292 & .1353 & -1375 & -1452 & -1541 \\
\hline (in percent of GDP) & .22 .1 & .18 .7 & -21.4 & -24.1 & -23.7 & $-23,9$ & -22.8 & -23.3 & -22.9 & -22.3 & -22.0 & -21.3 \\
\hline Cursent acoulun bylance & -195 & -272 & -274 & .263 & -410 & -440 & -386 & -471 & .443 & -482 & -487 & -498 \\
\hline (in percent of GDP) & -6.9 & -7.7 & .7 .1 & -6.2 & .8 .9 & -9.1 & -7.7 & -8.5 & -7.6 & .80 & -7.4 & -6.9 \\
\hline (in percent of GDP; incl. ofticial transfors) & -3.5 & -3.7 & -4.2 & -3.2 & -6.4 & -6.6 & -5.3 & $\cdot 6.1$ & -5.5 & -5.7 & -5.3 & -5.1 \\
\hline Official transfurs & 89 & 139 & 111 & 126 & 113 & 121 & 119 & 134 & $(2 t)$ & $1 \exists 8$ & 135 & 132 \\
\hline (in percent of GDP) & 3.2 & 3.9 & 2.9 & 3.0 & 2.5 & 21 & 2.4 & 2.4 & 2.1 & 2.3 & 2,0 & 1.8 \\
\hline Gross international neservess & 384 & 465 & 608 & 754 & 809 & 860 & 819 & 898 & 908 & 932 & $94 \hat{3}$ & 952 \\
\hline (in months of imports of goods und servicey) & 3.7 & 3.8 & 4.1 & 4.3 & 4.9 & 4.5 & 4.6 & 4.4 & 4.3 & 4,3 & 4.1 & 3.8 \\
\hline (relative to extermal dett service) & 14.9 & 19.6 & 22.1 & 21.6 & 12.6 & 14,7 & 12.9 & 16.0 & 11.9 & 12.9 & 12.0 & 9.5 \\
\hline (ii perucid of limsad maney) & 22.6 & 22.4 & 26.7 & 26.2 & 27.7 & 27.6 & 25.7 & 26.8 & 24.6 & 25.5 & 23,4 & $2 \mathrm{~L} . \mathrm{I}$ \\
\hline Change in resl effective exchange rate & 18.3 & 12.5 & 7.0 & 4.3 & $\cdots$ & -0.4 & $\cdots$ & $\cdots$ & $\cdots$ & $\cdots$ & $\cdots$ & $\cdots$ \\
\hline Memorandum item & & & & & & & & & & & & \\
\hline Nominal GDP (ïn millions of lek') $\mathrm{t}$ & 425,356 & 488,611 & $5.51,282$ & 611,622 & 658,062 & 637.272 & 718,574 & 739,048 & 786,007 & 810,276 & 890,340 & 976,100 \\
\hline
\end{tabular}

Sources: Albanian authorities; and Fund s1aff estimater and projcotions.

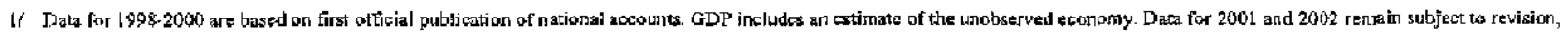
pending the compilation of official National Accounts.

2. Current netuvunt cxeludiug nfficial transfers.

3\% Revenue minus current expenditurs.

4. Grants are estimated, except for the 2002 outturn.

5/ Insluding interest payments for bank restucturing.

6ot [raluding bonds for bank restrueturing (lek 24.6 bn for 2000)

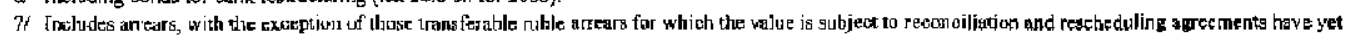
to be reached with creditors.

B/ For 1999 excluding ingorts of disect humanitarian aid related to the Kosovo crisis. 
Table 2. Albania: Quantitative Performance Criteria and Indicative Targets for 2001-04 1/

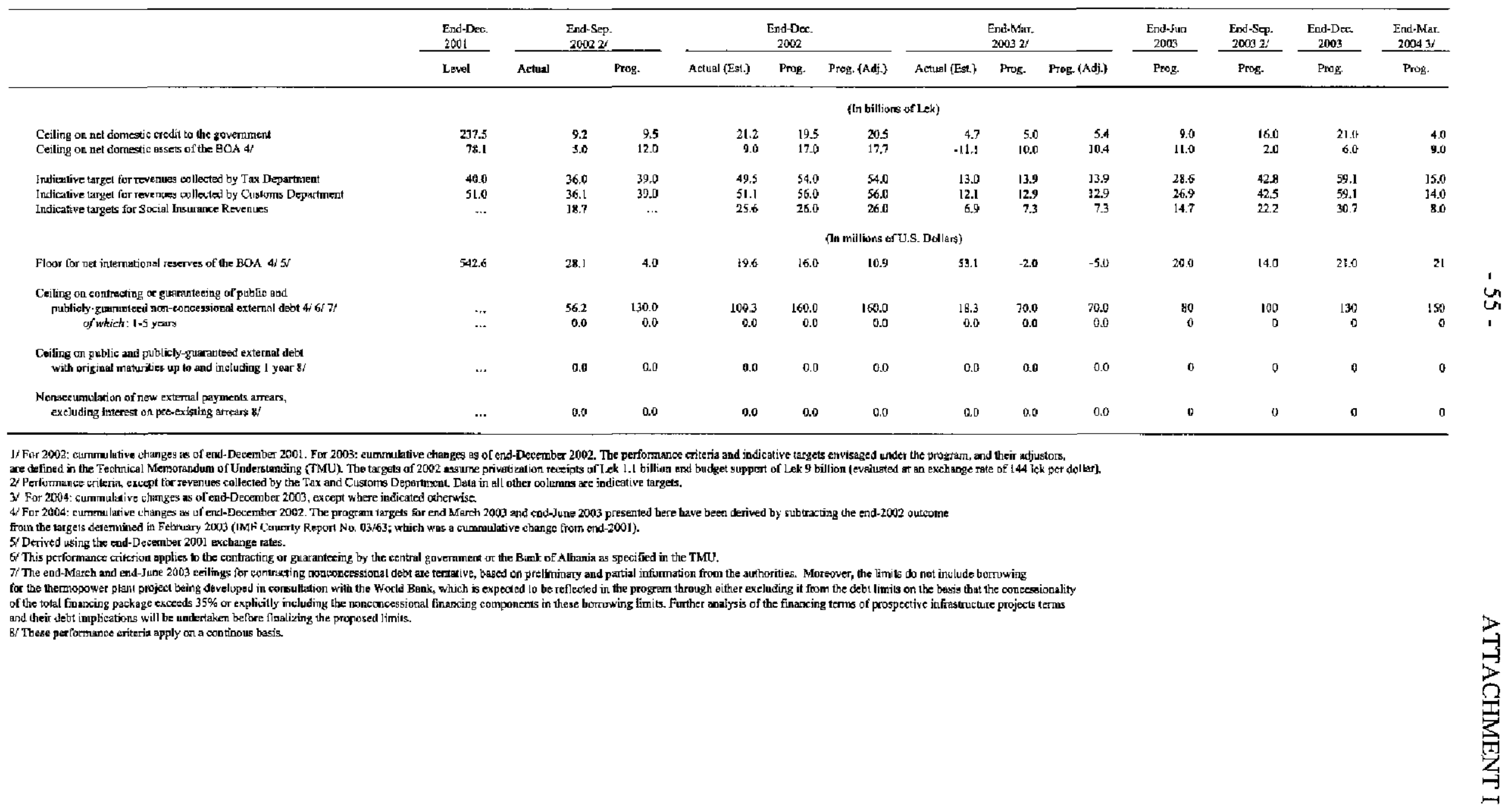

CInternational Monetary Fund. Not for Redistribution 
Table 3. Albania: Performance Criteria and Strustural Benchmarks under the PRGF Arrangement

\section{A. Prior Action}

Develop expenditure antingeneies for the second half of 2003 -in elose consultation with line trivistries and in line with the NSSED-and submit these to IMF staff (MEFP, ๆ13).

\section{B. Performance Criteria}

1. Execute the goventuent-assisted netting and final settlement of public sector inter-enterprise anears as of end-2001, and present. to Fund staff strategies for ensuring the full and tinely paysant of all obligations contracted thereafter (MEFP \& 13 ).

2. Complete implementation of ASYCUDA sontputer administration system in the Tirana custom house and Durrēs pert and unsure some allurnative means of dats transtrission until both systems are brought online and the transit systera pith ASYCUDA is fuily

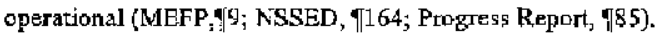

\section{Structaral Benchmarks}

1. Strengthen governance in relulion to the public administration by:

(i) extending the Civil Serviet Law to include all institutions related to govemunert spending agencies (MEFP, 118 ; NSSED, $\| 182$; Progress Report, 1 \&1).

(ii) starting the enforcement of procedutes to implement the law on the asset declaration of publiw officials developed in consultation with the World Bauk (MEFP, $118 ;$ NSSED, 1183; Progress Repart, F104).

(iii) (a) strengthening tax appeals procedures, including by publishing decisions of the tax appeals commissionj and (b) including instructions on tax procedures in the official gazette and in pamphlets distributed to taxpayers (MEFP, "10; NSSED, \$164; Progress Report, ๆ106\%.

3. Strengthen tax and eustoms administration through:

(i) a decision by the Council of Ministers to amend the structure of the Customs Department to establish a past-clearance control unil of around 10 senior inepectors concentrating an valuption, classification, origin, exımption, and suspension claims (MEFP, f9; NSSED, f164; Progress Report, ๆ85].

(ii) establishing at the Treasury a separate accounl for VAT refunds, fanded with uround 5 percent of gross VAT receipts already collected, and requiring the GDT to report on VAT refunds along with the rnonthly publication of data nn revenue collected (MEEP, १९; NSSED, ๆ198; Progress Repurt, ๆ85).

4. To prepare for privatization:

(i) settle Albtelekom's remaining thancial claims (MEFP, 920; NSSED, .266; Progress Report. $\| 132$ ).

(ii) transfer a turther part of the fiscal functions of the Savings Bank to private banks, by completing the auctioning of these functions in 5 cities (MEFP, 120 ; NSSED, 1207 ; Progress Report, ๆ122).

End -December 2003

End-December 2003

End-December 2003

End-July 2003

Throughout, starting end-June 2003

S. Regarding externul arrears:

(i) prepare quarterly reports (within one ruonth of the end of each quarter) on the slock of external arrears (MEFP, 125).

Throughout

(ii) submit to Parliament for ratification the protowbl agrements for restructuring of arrears with l(ungary (MEFE, ๆ 25). 


\section{ALBANIA \\ Technical Memorandum of Understanding}

This memorandum defines the quantitative benchmarks and performance criteria established in the Memorandum of Economic and Financial Policies for June 2003-March 2004 (MEFP).

\section{A. Net Domestic Credit to the Central Government}

1. For the purposes of the program, the central government covers the State Budget, the Social Security Institute (SSI), and the Health Insurance Institute (HII).

2. Net domestic credit to the government (NCG) is defined as the sum of domestic credits in lek and in foreign currency, except for onlending of foreign project loans to all parts of the central government as defined above, including Treasury bills and bonds held by the Bank of Albania, domestic commercial banks, and other domestic lenders (including insurance companies, other firms, and households), less the sum of central government deposits with the banking system (but excluding foreign currency deposits related to foreign financed projects), and the deposits of the SSI and the HII. ${ }^{1}$ Credits comprise bank loans and advances to the government (excluding advances on profit transfers by the Bank of Albania), holdings of government securities, due but unpaid interest, and negative balances in government deposits with banks.

3. The component of the domestic credit to government in the form of securities will be calculated based on data on their outstanding stock valued at issue price, with the adjustment for the amount held by the units of central government as defined above (in particular, the SSI and the HII). Sales of Treasury bills will be counted excluding the discount. Reported repayments of Treasury bills and other government securities will not include interest payments, either as coupon interest or the discount. Those components of net donestic credit to the government denominated in foreign currencies are to be valued as stipulated in the GFS. Data on other components of credit to government, if any, will be reported by the Bank of Albania.

4. According to the above definition, the level of net domestic credit to government was Lek 260.9 billion at end-December, $2002 .^{2}$ Gross loans were composed of (i) total outstanding T-bills at issue price in the amount of Lek 263.9 billion, of which

\footnotetext{
${ }^{1}$ Nonbank domestic lenders comprise both firms (including insurance companies) and households. For small lenders, a Treasury bill window is available at the central bank.

2 This amount differs from "claims on government (net of deposits)" in the standard monetary aggregates table, as the latter excludes nonbank lending and includes foreign currency deposits.
} 
Lek 72.7 billion was held by the Bank of Albania, Lek 176.3 billion by commercial banks, and Lek 14.8 billion by other domestic lenders; and (ii) other government lek securities, loans, and other claims on government in the amount of Lek 3.7 billion. From these gross loans, the following items were deducted: (i) central government deposits (excluding social security funds) in the amount of Lek 0.5 billion; and (ii) SSI and HII deposits and T-bill holdings in the amount of Lek 6.2 billion.

5. The limits on the change in net domestic credit to the government will be cumulative from end-December 2002.

\section{B. Net Domestic Assets}

6. The net domestic assets (NDA) of the Bank of Albania are defined as the difference between reserve money-defined as the sum of currency issue (less lek notes and coins held by the Bank of Albania) and commercial bank reserves held by the Bank of Albania-less the net international reserves of the Bank of Albania (Section C), with all foreign currency assets and liabilities valued in local currency for program monitoring purposes at an exchange rate at end-December 2001. Under this definition, the level of the NDA was Lek 87.1 billion as of end-December 2002. The NDA limits will be cumulative changes from end-December 2002 and will be monitored from the accounts of the Bank of Albania.

\section{Net International Reserves}

7. Net international reserves (NIR) are defined as reserve assets minus reserve liabilities of the Bank of Albania. Reserve assets are readily available claims of the Bank of Albania on nonresidents denominated in foreign convertible currencies, and held for the purpose of meeting balance of payments financing needs, intervention in exchange markets, and other purposes. They include Bank of Albania holdings of monetary gold, SDRs, Albania's reserve position in the IMF, foreign currency cash, and deposits abroad. Excluded from reserve assets are any assets that are pledged, collateralized, other otherwise encumbered; claims on residents; precious metals other than monetary gold; assets in nonconvertible currencies; illiquid assets; and claims on foreign exchange arising from derivatives in foreign currencies vis-à-vis domestic currency (such as futures, forwards, swaps, and options). Reserve liabilities shall be defined as foreign exchange liabilities to residents and nonresidents of the Bank of Albania, irrespective of their maturity. They include: foreign currency reserves of commercial banks held at the Bank of Albania; all credit outstanding from the IMF; commitments to sell foreign exchange arising from derivatives (such as futures, forwards, swaps, and options); and all arrears on principal or interest payments to commercial banks, suppliers, or official export credit agencies. Excluded from reserve liabilities are the government's foreign currency deposits at the Bank of Albania. ${ }^{3}$ Reserve assets and reserve liabilities will both be expressed in U.S. dollars.

${ }^{3}$ This exclusion is justified by current procedures in Albania, whereby the government's foreign currency receipts are deposited in a blocked account at the Bank of Albania and the 
8. During this program, for monitoring purposes, the exchange rates of the SDR and non-dollar currencies will be kept at their end-December 2001 levels and holdings of monetary gold will be valued at SDR 35 per ounce. Excluded from gross international reserves are holdings of nonconvertible currencies, claims on nonresident financial institutions denominated in nonconvertible currencies, and other claims which are not readily available.

\section{Adjusters for NCG, NDA, and NIR}

9. The NCG ceiling, NDA ceiling and NIR floor are defined on the assumption that privatization proceeds will amount, on a cumulative basis, from January 1, 2003, to:
End-September 2003
Lek 0.650 billion
End-December 2003
L.ek 1.077 billion

The NIR floor will be adjusted upward (downward) and the NDA ceiling adjusted downward (upward) by any excess (shortfall) in these receipts. The NCG ceiling will be adjusted downward by any excess in these receipts up to $1 / 2$ percent of GDP (Lek 3.7 billion). It will remain unchanged for any additional excess. The NCG ceiling will be adjusted upward by any shortfall in these receipts.

10. The NCG, NDA, and NIR targets are defined based on the assumption that foreign budgetary and/or balance of payments financing (excluding IMF financing and project and commodity loans or grants, or other aid) will amount, on a cumulative basis, from January 1, 2003, to:

\section{End-September 2003 US\$18 million \\ End December 2003 U\$\$21 million}

In cases when total foreign financing exceeds (falls short) this projection, the target for the net domestic credit to the government and the net domestic assets of the Bank of Albania will be adjusted downward (upward), and for the net international reserves upward (downward), with the proviso that the upward adjustment to the NCG and NDA ceilings and the downward adjustment to the NIR floor should not exceed US\$15 million ${ }^{4}$.

funds are transferred to the government's lek account before being spent. A change in this procedure, would require revisiting the NIR definition.

${ }^{4}$ For the NCG adjuster, the lek equivalent of deviations from the programmed amounts in terms of dollars is converted at an exchange rate of Lek 145 per U.S. dollar, in order to ensure consistency with the budget projections. 
11. The NDA ceilings will be also adjusted to reflect the impact of any change in the required reserve ratio of commercial banks with the Bank of Albania.

\section{E. External Debt and Arrears}

12. As set forth in point No. 9 of the Guidelines on Performance Criteria with Respect to Foreign Debt (Decision No. 12274-00/85) August 24, 2000), the term "debt" will be understood to mean a current, i.e., not contingent, liability, created under a contractual arrangement through the provision of value in the form of assets (including currency) or services, and which requires the obligor to make one or more payments in the form of assets (including currency) or services, at some future point(s) in time; these payments will discharge the principal and/or interest liabilities incurred under the contract. Debts can take a number of forms, the primary ones being as follows: (i) loans, i.e., advances of money to obligor by the lender made on the basis of an undertaking that the obligor will repay the funds in the future (including deposits, bonds, debentures, commercial loans and buyers" credits) and temporary exchanges of assets that are equivalent to fully collateralized loans under which the obligor is required to repay the funds, and usually pay interest, by repurchasing the collateral from the buyer in the future (such as repurchase agreements and official swap arrangements); (ii) suppliers' credits, i.e., contracts where the supplier permits the obligor to defer payments until some time after the date on which the goods are delivered or services are provided; and (iii) leases, i.e., arrangements under which property is provided which the lessee has the right to use for one or more specified period(s) of time that are usually shorter than the total expected service life of the property, while the lessor retains the title to the property. For the purpose of the guideline, the debt is the present value (at the inception of the lease) of all lease payments expected to be made during the period of the agreement excluding those payments that cover the operation, repair or maintenance of the property. Arrears, penalties, and judicially awarded damages arising from the failure to make payment under a contractual obligation that constitutes debt are debt. Failure to make payment on an obligation that is not considered debt under this definition (e.g., payment on delivery) will not give rise to debt.

13. The limit on medium- and long-term extemal debt applies to the contracting or guaranteeing by the central government or the Bank of Albania, of new nonconcessional external debt with an original maturity of more than one year, with sublimits on external debt with an original maturity of more than one year and up to and including five years. It applies not only to debt as defined in paragraph 12 of this memorandum, but also to commitments contracted or guaranteed for which value has not been received. External debt will be considered to have been contracted at the point the loan agreement or guarantee is ratified by the Albanian parliament. Excluded from the limits are refinancing credits and rescheduling operations (including the deferral of interest on commercial debt), credits extended by the IMF, and credits on concessional terms defined as those with a grant element of at least 35 percent. The grant element is to be calculated using the OECD Commercial Interest Reference Rates (CIRRs): for maturities of less than 15 years, the grant element will be calculated based on six-month averages of CIRRs; and for maturities longer than 15 years, the grant element will be calculated based on ten-year averages. Debt falling within the limit 
shall be valued in U.S. dollars at the exchange rate prevailing at the time the contract or guarantee becomes effective.

14. The limit on short-term external debt applies on a continuous basis to the stock of short-term external debt owed or guaranteed by the central government or the Bank of Albania, with an original maturity of up to and including one year. It applies to debt as defined in paragraph 12 of this memorandum. Excluded from the limit are rescheduling operations (including the deferral of interest on commercial debt) and normal import-related credits. Debt falling within the limit shall be valued in U.S. dollars at the exchange rate prevailing at the time the contract or guarantee becomes effective.

15. A continuous performance criterion applies to the nonaccumulation of new external payments arrears on external debt contracted or guaranteed by the central government or the Bank of Albania. External payment arrears consist of external debt service obligations (principal and interest) falling due after March 31,2002 and that have not been paid at the time they are due, as specified in the contractual agreements. Excluded from the prohibition on the accumulation of new arrears are: (i) arrears arising from interest on the stock of arrears outstanding as of March 31,2002; and (ii) external arrears that are subject to debt rescheduling agreements or negotiations.

\section{F. Tax Revenues}

16. Collection of revenues by the Tax and Customs Departments and social insurance contributions will be monitored on the basis of quarterly indicative floors.

\section{G. Monitoring and Reporting Requirements}

17. Performance under the program will be monitored from information supplied monthly to the Fund by the Bank of Atbania and the Ministry of Finance. The following information will be communicated monthly to the Fund by the Ministry of Finance: the summary fiscal table, including the overall budget deficit, on a cash basis; their issuance of Treasury bills including gross value and cash received; and privatization receipts. The Bank of Albania will communicate monthly to the Fund: the balance sheets of the Bank of Albania, and the consolidated accounts of the commercial banks; the monetary survey; the net domestic credit to the government; the NFA of the Bank of Albania; the foreign exchange cashflow of the Bank of Albania, including the level of official reserves; daily average exchange rates; and trade flows. The following information will be communicated quarterly to the Fund by the Ministry of Finance: information on the contracting and guaranteeing of new debt; information on the stock of short term, and on medium- and long-term debt; and, with assistance from the Bank of Albania, information on all overdue payments on short-term debt and on medium- and long-term debt. The Bank of Albania will also communicate to the Fund periodic updates of balance of payments estimates. 


\section{Statement by the IMF Staff Representative on Albania \\ July 2, 2003}

This statement describes developments since the staff report was issued on June 18.

As a prior action for this review, and in view of ongoing revenue shortfalls, the Albanian authorities have now completed the preparation of contingency plans for containing expenditures during the remainder of 2003. The details of these plans were communicated to staff in two letters by Finance Minister Islami, dated June 13 and June 24. The plans include reductions in subsidies for electricity imports and in operations and maintenance expenditures, but protect spending in priority areas.

Staff is planning a visit in mid-July to initiate discussions on the 2004 budget and to assess fiscal performance so far this year. If it finds that revenue shortfalls are likely to continue, it will call for the immediate activation of the contingency plans. 


\section{IMF Completes In Principle Second Review and Approves US\$6 Million Loan under PRGF for Albania}

The Executive Board of the International Monetary Fund (TMF) today completed, in principle, the second review of Albania's economic performance under its Poverty Reduction and Growth Facility arrangement. The IMF Board's decision will become effective upon a further decision following the World Bank's Executive Board review of Albania's Poverty Reduction Strategy paper (PRSP), scheduled for July 10, 2003. At that time, Albania will be able to draw SDR 4 million (about US\$6 million) from the IMF.

The three-year PRGF arrangement was approved on June 21, 2002 (see News Brief No. 02/52) for a total of SDR 28 million (about US\$39 million). So far, Albania has drawn SDR 8 million (about US\$11 million) under its current PRGF arrangement from the IMF.

The PRGF is the IMF's most concessional facility for low-income countries. It is intended that PRGF-supported programs will in time be based on country-owned poverty reduction strategies adopted in a participatory process involving civil society and development partners, and articulated in a Poverty Reduction Strategy Paper (PRSP). This is intended to ensure that each PRGF-supported program is consistent with a comprehensive framework for macroeconomic, structural, and social policies to foster growth and reduce poverty. PRGF loans carry an annual interest rate of 0.5 percent, and are repayable over 10 years with a $51 / 2$-year grace period on principal payments.

Following the Executive Board discussion, Anne Krueger, First Deputy Managing Director and Acting Chair, said:

"Albania"s performance during the first year of the three-year PRGF-supported program on balance has been satisfactory, with low inflation, a stable effective exchange rate, and gradual fiscal consolidation. However, structural reform has been slower than envisaged; the authorities have taken steps to speed up the process and strengthen their economic strategy. The Fund has concluded the second review under the program, on the strength of the authorities' commitment to and ownership of the program, the progress being made in the fiscal area, and the strong and well-sequenced structural reform agenda. 
"Fiscal policy has been hindered by weaker-than-expected revenue collection, but the deficit targets have been met. Looking forward, strong measures have been identified to broaden the tax base, improve revenue collection, and strengthen budgetary procedures. These should permit both increased expenditures on priority measures for poverty alleviation and continued fiscal consolidation. In the context of low core inflation and continued exchange rate stability, there is scope for further lowering policy interest rates.

"The steadfast implementation of structural reforms remains crucial to high private-sector led growth. Key priorities are removing administrative barriers to investment, improving governance, and fighting corruption.

"The authorities have developed a first progress report on their comprehensive National Strategy for Socio-Economic Development. This document testifies to the authorities' commitment to their poverty reduction strategy, which continues to provide a suitable framework for the current policy program and for the support of the international community," Ms. Krueger stated. 


\section{Statement by Pier Carlo Padoan, Executive Director for Albania \\ July 2, 2003}

At the outset I would like, on behalf of my Albanian authorities, to express our appreciation and to thank Staff for the very fruitful consultations and for preparing a comprehensive set of documents, which provide a candid assessment of the program and the challenges ahead in the implementation of economic and structural reforms.

The Authorities broadly concur with the key findings of the reports and would like to reiterate their firm commitment to maintaining macroeconomic stability and to making further progress in speeding up structural reforms. While authorities fully recognize the importance of a stable political environment for the success of the program they feel that concerns about the consequences of the upcoming electoral period are overstated. The policy framework offered by the agreement with the Fund is widely recognized as necessary for Albania to progress in macroeconomic stabilization, to sustain growth and to reduce poverty.

\section{The macroeconomic outlook}

The macroeconomic outlook remains favorable in spite of growth deceleration, which is mainly due to an unfavorable external environment. The Authorities are aware, however, that sustained growth cannot be achieved without a significant strengthening of the business climate and the elimination of major supply constraints, especially in the energy sector.

Prudent monetary policy implementation has kept inflation under control and is making room for careful monetary easing against the background of renewed stability of the banking sector. Progress is also being achieved in monetary management, with increased reliance on market practices. Authorities are benefiting from advice by MFD and LEG.

The external position remains manageable, also given the still-satisfactory expected FDI inflows, and external debt remains sustainable. Of course, sustained FDI inflows will require continuing progress in strengthening the business environment and full implementation of the reform program.

\section{Fiscal policy}

Fiscal balances have broadly remained within the program targets. However the Authorities are fully aware that this is the result of both a weak revenue performance and of expenditure cuts, and they are taking necessary measures to strengthen tax administration and improve expenditure prioritization. Authorities are implementing the measures agreed under the program to strengthen revenue collection (which are themselves a source of additional expenditure). The expected positive outcomes, however, will take some time to materialize. This notwithstanding, revenue collection remains exposed to downside risks.

Authorities agree with staff that, given such downside risks, respecting fiscal targets will require expenditure cuts that will have to be carefully prioritized so as to protect critical items such as health care and education. A contingency plan laying out measures for the 
remainder of the year has been prepared as a prior action for this review. The plan includes savings in administrative expenditures, reduction in subsidies for electricity imports, and reductions in the public wage bill.

The budget implementation process will also be improved in the context of the 2004 budget.

\section{Strengthening governance and improving the business environment}

The Board discussion in occasion of the completion of the first review had put a strong emphasis on strengthening governance and fighting corruption. These remain among the Government's top priorities. Since the time of the first review under the PRGF, considerable progress has been made in improving the regulatory framework and enhancing the transparency and accountability of public institutions as well as the transparency and communication between the administration and the business community. Actions have been taken to correct hiring procedures of public officials, which had raised concerns.

The business climate is visibly improving. Agencies dedicated to the support of small business and to the attraction of FDI inflows are being created. These will be operated according to principles of efficiency in service production and their size will be kept at a minimum.

\section{Structural reforms and the energy sector}

The Authorities are committed to speeding up structural reforms, which are at the core of the Government's agenda. They are aggressively implementing measures to improve business environment, strengthen the reform in the energy sector, and finalize the privatization of Albtelecom and of the Savings Bank for which authorities will again contact potential buyers

A lasting solution to the problem of energy shortages will require the gradual elimination of dependency from foreign sources of supply. Authorities are concerned that, in the transition to full independence in energy production and distribution, meeting targets related to power supply will require that power availability to households and industry be often severely reduced. This produces negative effects both on the level of activity and on the degree of business confidence, both harmful to growth. Authorities are confident that, with the financial support of a soft loan from the Italian government, the restructuring of KESH will proceed as scheduled.

\section{Reducing Poverty}

Reducing poverty and inequity of opportunities remain a great challenge for the country. My Authorities fully agree with the JSA Progress Report and are committed to sustain the intensive efforts in order to enhance the efficient implementation of the National Strategy for Socio-Economic Development. 